\title{
Global Warming, Global Warming Pause and the Role of $\mathrm{CO}_{2}$
}

\section{Giora Shaviv ${ }^{* \dagger}$}

Dep. of Physics, Israel Institute of Technology, Haifa, Israel

E-mail: gioraeshaviv.org

We discuss several aspects of the phenomenon called Global Warming and refrain from any conclusions. We leave the reader to draw his conclusions about the series of observations presented here.

The phenomenon called Global Warming or in the new name Climate Change, refers to the behavior of the 'Temperature of the Earth' as a function of time. The 'temperature of the Earth' is a complicated term as it is measured in different locations and one has to define at what location we discuss the temperature and how we average the temperature over the complicated surface of the Earth so as to obtain a single number. The atmosphere, or better said the troposphere, can be considered as a boundary layer. However, this layer is not isothermal and shows large temporal and location temperature variations. There are three basic questions the data should point to:

- How the "Global Temperature" of the Earth is evaluated and how the statistical mean behaves as a function of time,

- How the temperature varies at a given fixed location, $\mathrm{T}(\mathrm{t})$,

- What is the role of $\mathrm{CO}_{2}$ in driving the "Global Earth temperature".

We examine in the paper the following topics:

- The Global Earth Temperature and Definition of the Temperature Anomaly,

- The rise of the oceans,

- The behavior of the $\mathrm{CO}_{2}$,

- The acidity of the seas,

- Underwater volcanos,

- The dependence of $T(t)$ for a single place,

- Theoretical predictions of the Global Temperature,

- The Interaction of the Sun with Earth's climate,

- The phenomenon called 'Global Warming Pause.

in an attempt to provide a comprehensive answer to the above questions.

Accretion Processes in Cosmic Sources, APCS2016

5-10 September 2016,

Saint Petersburg, Russia

\footnotetext{
* Speaker.

${ }^{\dagger}$ This research was supported by the Ministry of Science, Israel.
} 


\section{The Global Earth Temperature and definition of the Temperature Anomaly}

The first to attempt to calculate the global Earth temperature was carries out by Fourier ${ }^{1}$ as early as 1824 , who wrote that "The question of terrestrial temperature, one of the most remarkable and difficult in natural philosophy, involves very different elements which require to be considered in a general light". Indeed, calculating the Global Earth temperature is also a fundamental and very debatable problem today.

The term 'temperature anomaly' means the departure from a reference value or a long-term average. A positive anomaly indicates that the observed temperature was warmer than the reference value, while a negative anomaly indicates that the observed temperature was cooler than the reference value. The temperature anomaly is measured routinely at numerous points on the Earth. Different points on Earth have different temperature anomalies at different times.

The Temperature Anomaly can be considered as a global-scale climate diagnostic tool and provides the big picture overview of average global temperatures compared to a reference value. Obviously, it is a statistical quantity.

Land surface temperatures are available from the Global Historical Climate Network-Monthly $(\mathrm{GHCN}-\mathrm{M})^{2}$. Sea surface temperatures are determined using the extended reconstructed sea surface temperature (ERSST) analysis. ERSST uses the most recently available International Comprehensive Ocean -Atmosphere Data Set (ICOADS) ${ }^{3}$ and statistical methods that allow stable reconstruction using sparse data. In other words interpolations and extrapolation of missing data.

The monthly ground temperature analysis begins January 1854 , but due to very sparse data, no global averages are computed before 1880 . With more observations after 1880 , the signal is expected to be stronger and more consistent over time.

Fig. 1 shows the temperature anomaly for the year 2015 as provided by NASA ${ }^{4}$. One clearly sees that in the year 2015 the Northern part of the Earth heated more than the Southern part and that the Earth does not heat (nor cool) uniformly. Moreover, there are even cold spots inside hot regions. If the problem were a simple heat transfer problem this would have required a strong cooling agent. Also note that the figure provides the data averaged over the entire year. However, the changes do not take place simultaneously over the entire Earth, namely the temperature may rise at a given location today and tomorrow it can rise at another location. These two independent changes are shown on the map as simultaneous. Actually, the cooling and heating are apparently controlled by the local/global flows of air and water currents much more than by global atmospheric agents.

The International Panel for Climate Chance (IPCC) argues that these global temperature changes are unprecedented. 'Recent climate changes have had widespread impacts on human and natural systems. Observed changes in the climate system namely, the climate system are unequivocal, and since the 1950s, many of the observed changes are unprecedented over decades to millennia. The

\footnotetext{
${ }^{1}$ The English translation in American Journal of Science by Burgess, E. (1837) of J-B. J. Fourier (1824) paper 'On the Temperatures of the Terrestrial Sphere'.

${ }^{2}$ The Climate Monitoring at NOAA's National Centers for Environmental Information.

${ }^{3}$ The data is collected from ship data, buoy data, coastal-marine automated data, miscellaneous and tide gauge stations.

${ }^{4}$ Note the continuity in the Land to Sea transition.
} 
atmosphere and ocean have warmed, the amounts of snow and ice have diminished, and sea level has risen'.

Furthermore, 'Each of the last three decades has been successively warmer at the Earths surface than any preceding decade since 1850 . The period from 1983 to 2012 was likely the warmest 30-year period of the last 1400 years in the Northern Hemisphere. The globally averaged combined land and ocean surface temperature data as calculated by a linear trend show a warming of $0.85{ }^{\circ} \mathrm{C}$ over the period 1880 to 2012, when multiple independently produced datasets exist'.

'In addition to robust multi-decadal warming, the globally averaged surface temperature exhibits substantial decadal and interannual variability. Due to this natural variability, trends based on short records are very sensitive to the beginning and end dates and do not in general reflect long-term climate trends ${ }^{5}$. This means that small number statistics cannot be trusted.

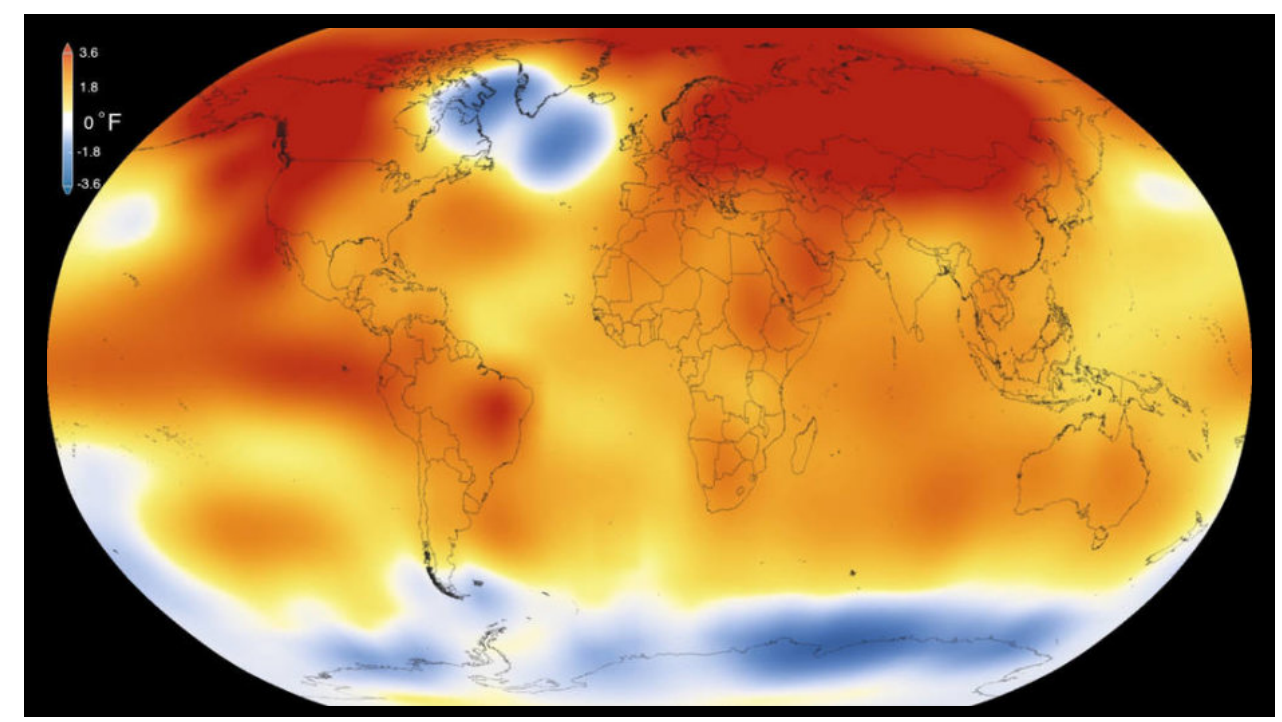

Figure 1: The temperature anomaly in 2015, the year NASA claims 'was the warmest year since modern record-keeping began in 1880', according to a new analysis by NASA's Goddard Institute for Space Studies. The claim is that the record-breaking year continues a long-term warming trend - 15 of the 16 warmest years on record have now occurred since 2001.Credit: Scientific Visualization Studio/Goddard Space Flight Center.

Note that the temperature anomaly is statistical and hence the actual values are averaged over a sufficiently long period say, a year. So while the value may be the same, the times at which the temperature rises/falls may be different. Again, the temperature did not rise in the entire area colored with a given color at the same time. Different places became warmer at different times. The temperature map look like global mixing on a scale of about $R_{\text {Earth }} /(20-30)$.

In fig. 2 we see the ground annual mean temperature of the Earth. This is the base temperature distribution against which the temperature anomalies are measured. As can be seen, the base temperature extends over about 80 degrees centigrade. So the $\approx 1^{\circ} \mathrm{K}$ per 80 degrees centigrade is equivalent to a negligible change. The data is too meager for a confident claim.

A crucial question is at what height is the temperature measured and how do you handle mountains which are above sea level and valleys that may be below sea level. We point out that the

\footnotetext{
${ }^{5}$ (The IPCC report 2015, Climate Change 2014 Synthesis Report, Summary for Policymakers).
} 

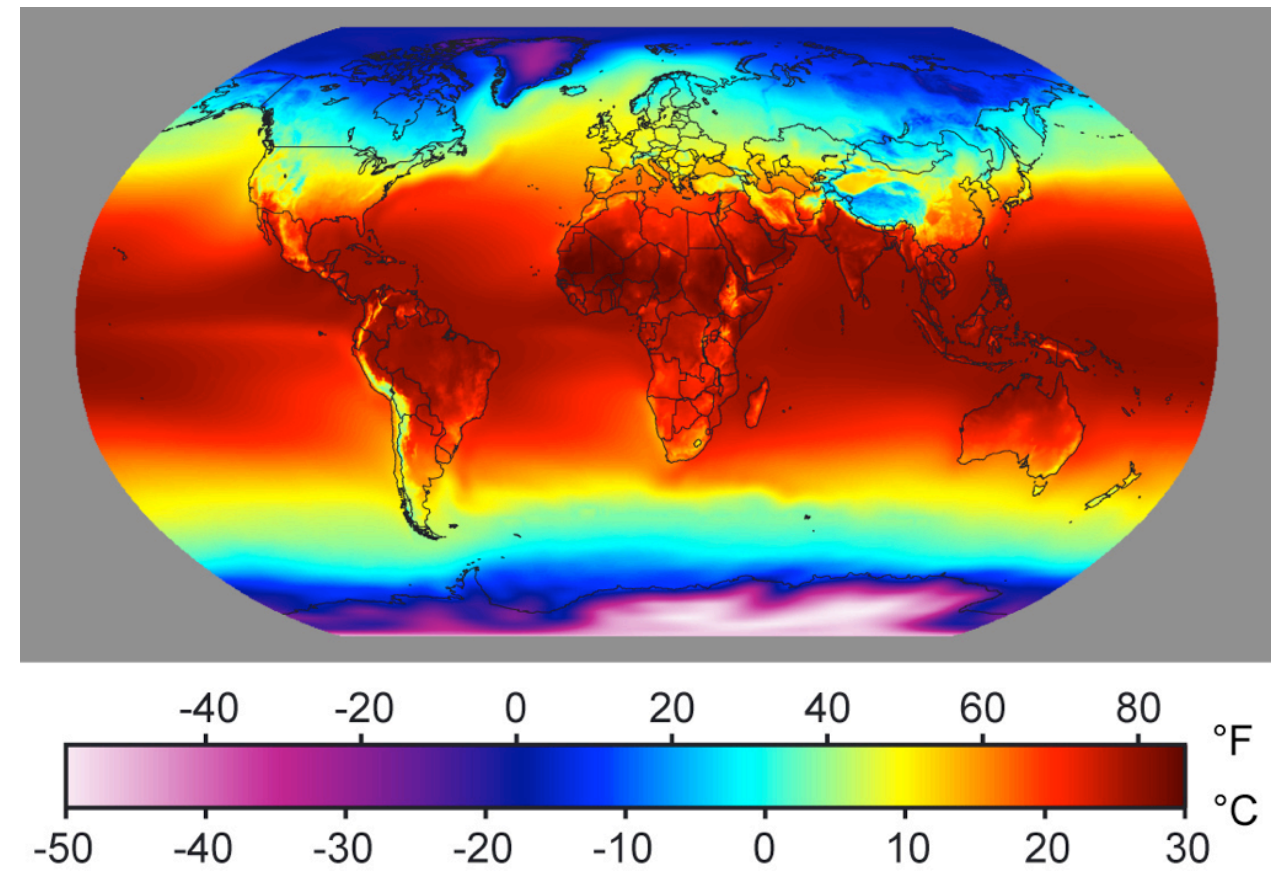

Ground Annual Mean Temperature

Figure 2: Global distribution of annual mean temperature near sea level. Source: NOAA-CIRES Climate Diagnostics Center, Boulder, Colorado.

standard height of weather stations is 10 meters. The sea temperature is the temperature at say, a few meters into the sea and not at 10 meters above the water. Land temperature is 10 meters above the ground and not a few meters in the ground. Strictly speaking, the air temperature is measured near the ground where the gradient is the largest. Hence, in many cases, when the ground is not at sea level a certain prescription is required to normalize the temperature to the same height above sea level. There are various algorithms for 'flattening the Earth' giving naturally different results.

Fig. 3 shows the temperature anomaly in the troposphere. The troposphere is extended over few kilometers and it is not clear where is the measurement carried out. A comparison between this figure and fig. 1 shows a substantial difference both in variability and in value. The lower troposphere has smaller changes in temperature relative to the surface level. This imply that the algorithm for 'flattening the Earth' is very crucial and controls the result.

The present day distribution of weather stations is shown in fig. 4. About 14,000 weather stations operate today. There were in the past many more stations, up to 250,000 , that were dropped from the network because of different reasons. The question about the effect of the dramatic decrease in weather station arose and least square fits to the global data (1880-2010) shows that full data from all station yields a temperature change of $0.59^{\circ} \mathrm{K} /$ century while the data after the elimination of most of the weather stations is $0.48^{\circ} \mathrm{K} /$ century ${ }^{6}$ and 'this difference in negligible'. The difference between the two results is mainly due to measurements before 1920 .

The Global Historical Climatology Network (GHCN) is a data base of temperature, precipita-

\footnotetext{
${ }^{6}$ https://www.skepticalscience.com/print.php?r=131
} 


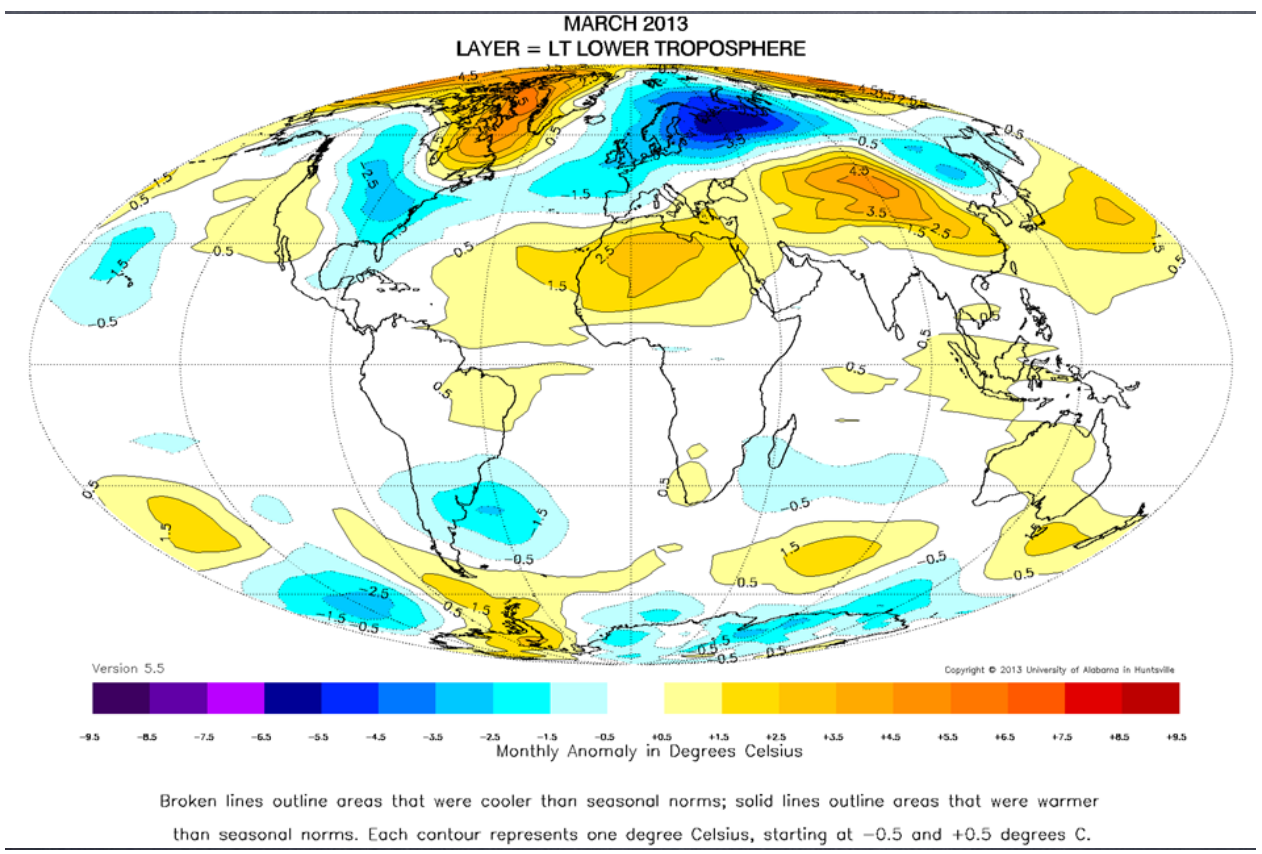

Figure 3: The temperature distribution in what is called the 'lower troposphere'. Source: Global Temperature Report, The University of Alabama in Huntsville, 2016.

\section{Global Climate Network Temperature Stations}

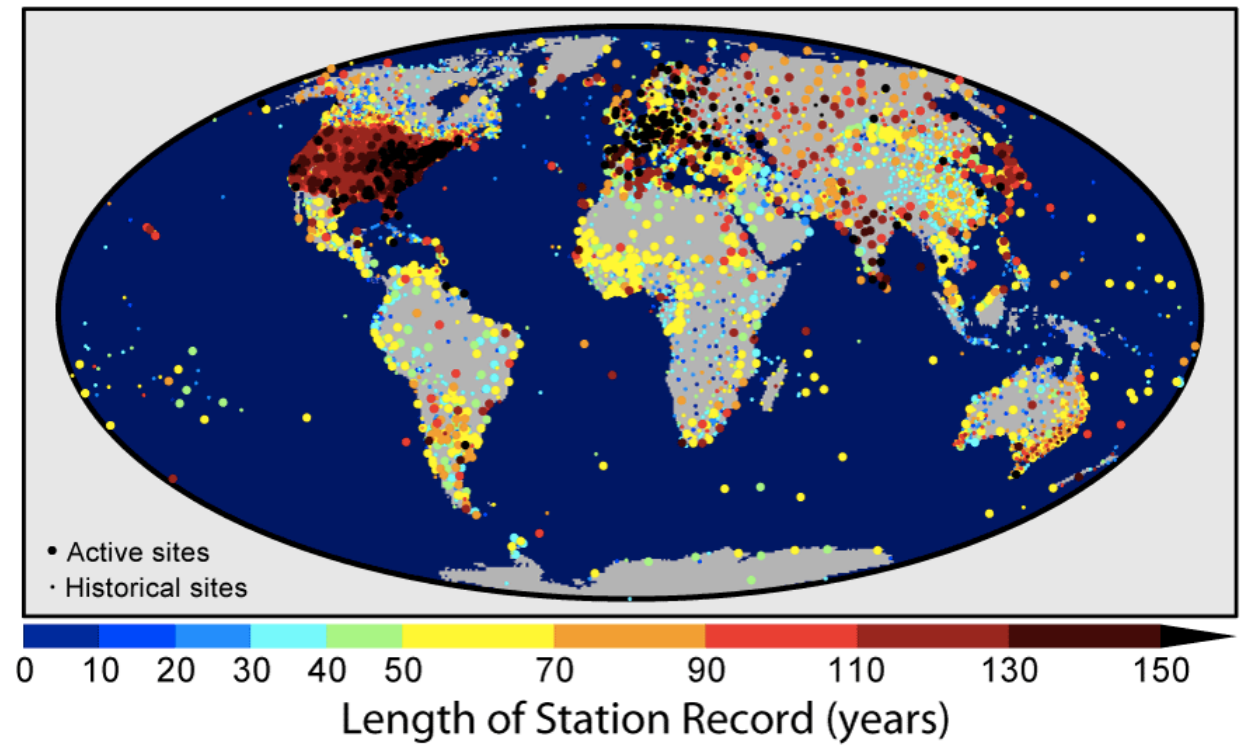

Figure 4: The distribution of weather stations used to obtain the temperature anomaly. The station are colored according to the length of the operation time of the station. 14,000 stations are marked. 
tion and pressure records managed by the National Climatic Data Center, Arizona State University and the Carbon Dioxide Information Analysis Center ${ }^{7}$.

The aggregated data are collected from many continuously reporting fixed stations on the Earth's surface and represent the input of approximately 6000 temperature stations, 7500 precipitation stations and 2000 pressure stations.

The above work has often been used as a foundation for reconstructing past global temperatures, and was used in previous versions of two of the best-known reconstructions, that prepared by the National Climatic Data Center (NCDC), and that prepared by NASA at its Goddard Institute for Space Studies (GISS) temperature set ${ }^{8}$. The average temperature record is 60 years long with about 1650 records greater than 100 years and about 220 greater than 150 years (based on GHCN v2 in 2006). The earliest data included in the database were collected in 1697.

We learn the following: About 1600 weather stations out of the 14,000 active today, operate in the USA. A somewhat smaller number operates in Europe. The rest of the world has a significantly lower density of measuring stations than the two major power consumers ${ }^{9}$.

About $2 / 3$ of the surface of the Earth is oceans and hence not covered by the above network.

The USA produces about $25 \%$ of the world energy consumption. Most of the weather station concentrate in regions where most of the world energy is consumed and emitted to the atmosphere.

As can be seen from fig. 4, the poles, central Africa, most of the Amazon and China and Manchuria are not covered by weather stations. Data in Antarctica was not taken till the nineteen fifties.

The measuring stations appear at all heights above sea level and their original data requires corrections. Needles to stress, the stations are not spread on the surface of the Earth in a distribution which maximizes accuracy, the locations are completely independent. Even in the USA the stations are not evenly distributed and interpolation between stations is needed.

There are large areas in the Arctic without weather stations. NASA GISS approaches the problem by filling in gaps with data from the nearest land stations. The Climatic Research Unit at the University of East Anglia, which works jointly with the Met Office Hadley Centre, leaves much of the region out of its global temperature analysis ${ }^{10}$.

There exist several organizations which attempt at calculation the Earth's temperature, the Met Office and the NCDC for example, leave areas of the Arctic Ocean without stations, while GISS approaches the problem by filling in the gaps with data from the nearest land stations, up to a distance of 1200 kilometers away. In this way, the GISS analysis achieves near "total coverage" in the Arctic.

To perform the evaluation of the Global Temperature the surface of the Earth is divided into small squares. The size of the squares varies and the smallest ones are $1 \mathrm{~km}$ by $1 \mathrm{~km}$. As there may

\footnotetext{
${ }^{7}$ Peterson, Thomas C. and Russell S. Vose (1997). "An overview of the Global Historical Climatology Network temperature data base". Bulletin of the American Meteorological Society. 78 (12): 2837-2849.

${ }^{8}$ GISS Surface Temperature Analysis (GISTEMP), National Aeronautics and Space Administration (NASA)/Goddard Institute for Space Studies (GISS) and retrieved May 9, 2014.

${ }^{9}$ Any utilization of power requires emission into the colder atmospheric heat reservoir and this causes heating like the Urban Heat Island

${ }^{10}$ NASA Earth Observatory/Robert Simmon.
} 
not be a station in every such square, for example in the Himalaya mountains many interpolations are called for.

What the accuracy of the calculation can be? There are on the Earth about $3.65 \times 10^{8} \mathrm{~km}^{2}$ of land but there are only $1.4 \times 10^{4}$ weather stations. Add to this the fact that $2 / 3$ of the surface of the Earth is covered with oceans and you find out that most of the squares have no weather station and need interpolation. Hence massive extrapolations and interpolations are required.

We see in fig. 5 the departure from the average temperatures in January to February 2015. This appears to be a cold winter in a slightly higher latitude then the Eastern parts of the USA but not in Europe. Also, Siberia warmed up and it is at roughly the same latitude as the the Eastern coast of the USA which became colder. Should we claim that local climate drivers overcame the $\mathrm{CO}_{2}$ heating in certain location but not in others or it is all a matter of local conditions? Note that the gray zones near the poles imply no weather stations. A quite different picture is obtained in

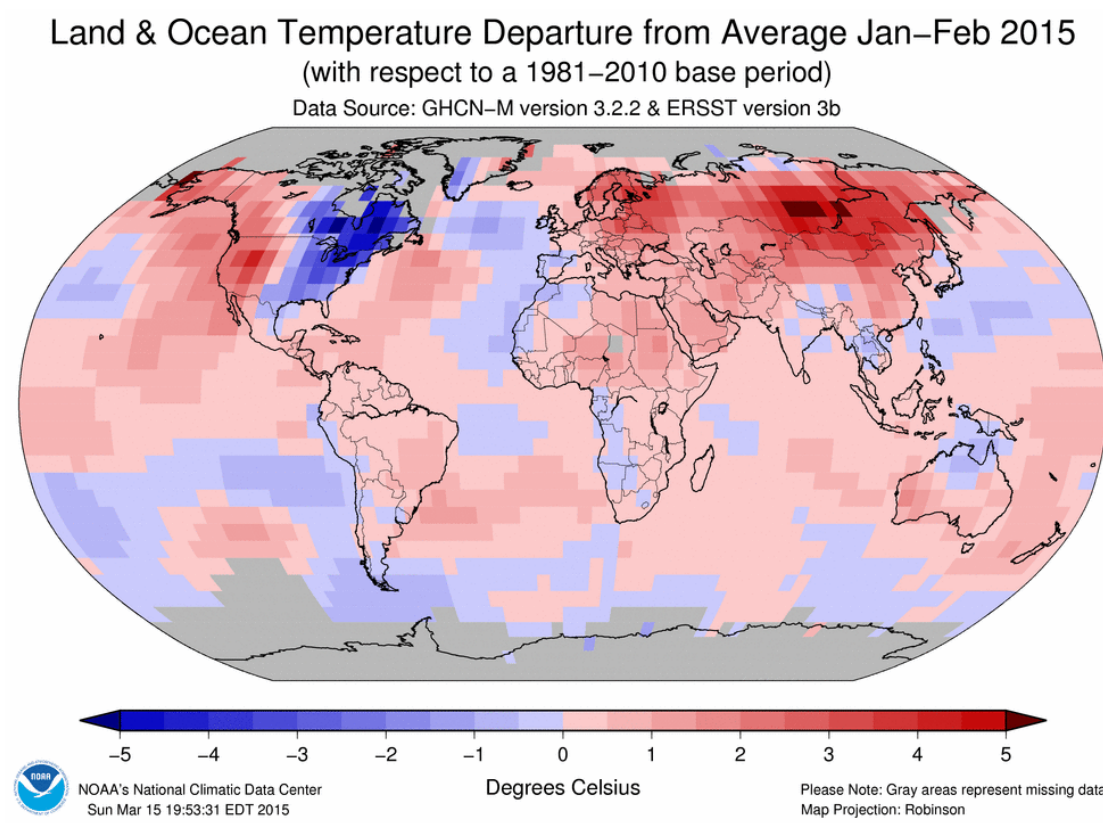

Figure 5: The Land \& Ocean combined to yield the Global Temperature Departure from Average in January - February 2015.

the same year but for the month of November consult fig. 6. Some of the regions reverse their response, what heated in one month cools in the other and vice versa. We do not see a persistent trend.

We show in fig. 7 the difference between the troposphere and the stratosphere. The stratosphere cools while the troposphere heats. One would have expected the inverse because the ozone absorbs heat and gives rise to a monotonically rising with height temperature in the stratosphere. However, the Global Temperature does not seem to follow this logic.

Is it possible that the temperatures measured from space in the stratosphere have some intrinsic bias towards lower temperatures? With the scattered and far from complete Earth coverage with weather stations one raised the idea to measure the temperature from satellites. This has been 


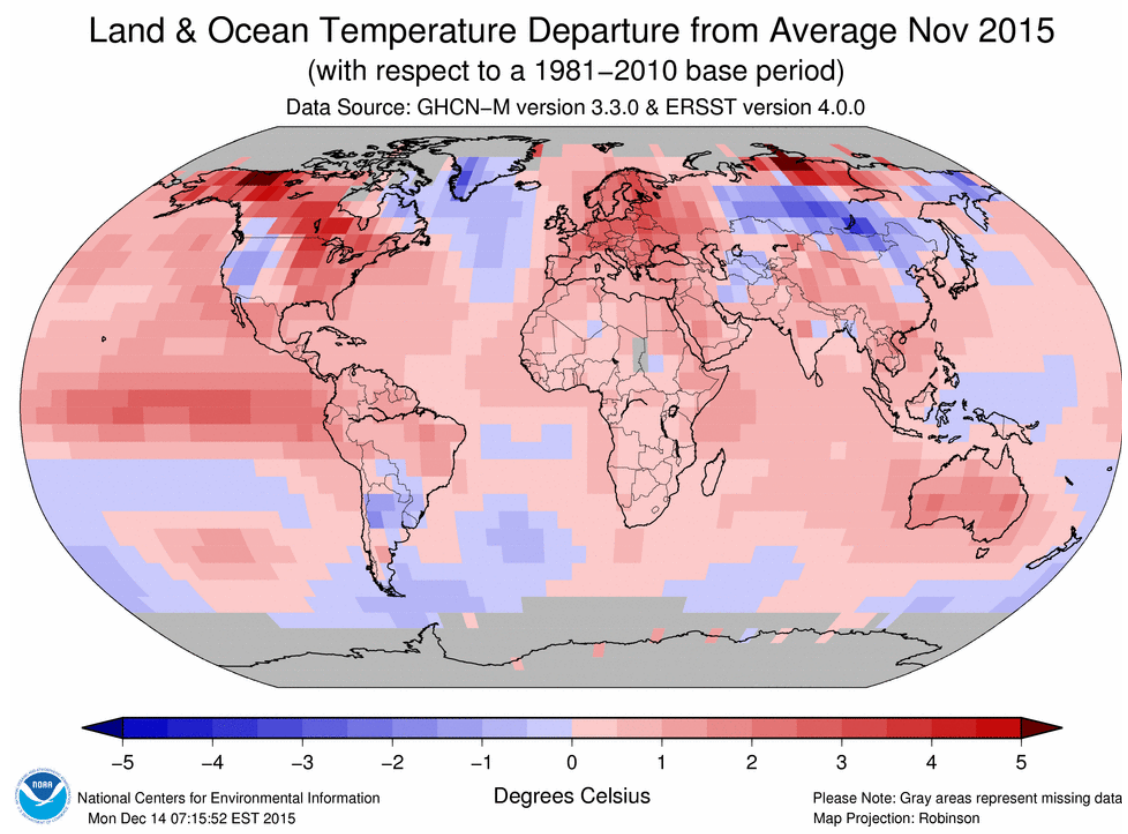

Figure 6: The Land \& Ocean combined to yield the Global Temperature Departure from Average in November 2015. There are no measurements in Antarctica and the North pole.

accomplished with NASA satellites and the amazing thing found was that satellite measurements were consistently lower than ground base ones.

One reason for the above problem is the difference in reference temperature the other in the algorithms implemented to reduce the satellite data. It is not clear whether the difference between ground and satellite based measurements has been fully accounted for. But if the difference in measurements between the ground and space are not fully accounted for why bother on measuring temperatures from space? What about other measuring from a distance astronomical observation carried out by astronomers?

There exists additional mysteries. For example, newly analyzed NASA satellite data from east Antarctica has set a new record for the coldest temperature ever recorded: $-94.7^{\circ} \mathrm{C}$. It happened on August 2010. Does it mean that the hottest year contained also the record coldest temperature on Earth?

We discussed above the Earth surface temperature however, it is of interest to examine what happens in other heights in the atmosphere and the sea-surface interface. Does only the surface of the Earth heat or the 'entire' atmosphere which is exposed to the Sun heats. In fig. 7 we compare the temperatures in the troposphere (the layer close to the surface of the Earth) with the temperature of the lower stratosphere ${ }^{11}$ and see to our surprise practically no difference between sea surface and land surface. The differences are remarkable, while the troposphere heats the stratosphere cools. This is clearly an unaccounted for radiative transfer-hydrodynamic effects in the atmosphere.

The variations of the temperature anomaly with time can be seen in the forthcoming global

\footnotetext{
${ }^{11}$ The region of the atmosphere above the troposphere and below the mesosphere. Near the equator it starts at $18 \mathrm{~km}$ and ends at $50 \mathrm{~km}$
} 

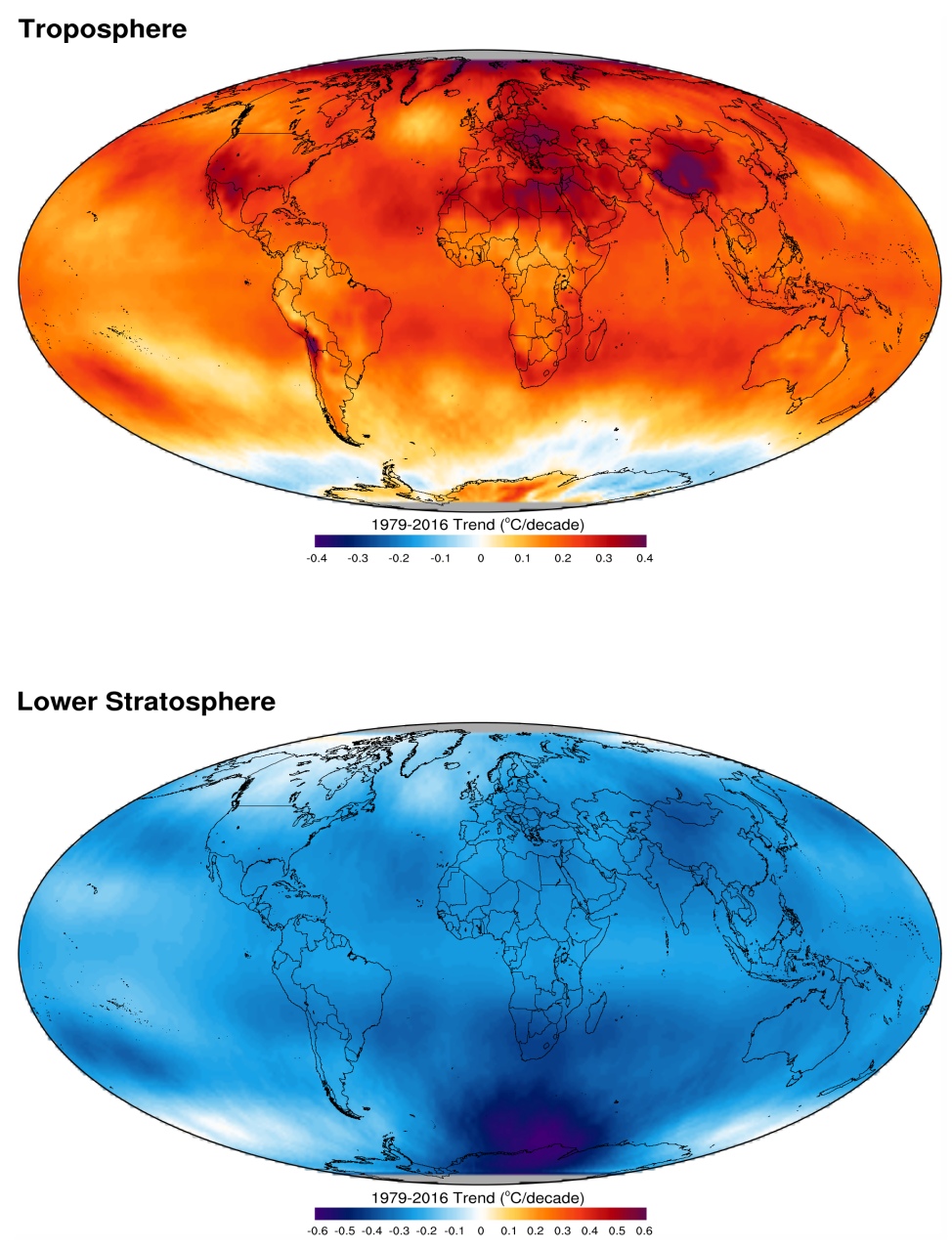

Figure 7: A comparison between the temperature in the troposphere and that in the lower stratosphere for the years 1979-2013. 
maps fig. 8 to 18 . The maps show large variations from square to square and it is not that few squares carry the lion share of heating or cooling. Every decade a different zone shows the largest variations. The Earth does not heat or cool as a uniform body, actually far from it and demonstrates that local weather is in dominates over global trends.

Observing the sequence of maps we see the following: during the first 30 years most of the temperature change took place in the Northern pole and it was cooling. Little took place in the Southern hemisphere. If you attempt to 'understand' this fact by claiming that the poles heat by the Hadley cells that extend between the equator and the poles then note that the change was cooling (not heating).

The change in the poles diminished almost to zero in 1935-1944, when we experienced the WWII. A small change in the poles is observed from that time till 1994 when it started to show heating (but mostly near the Northern pole.)

The ocean show some heating but not a uniform one. The difference in $\mathrm{CO}_{2}$ concentration around the globe is just 1-2\% (see later) so most of the heating due to changes in $\mathrm{CO}_{2}$ concentration should have taken place near the equator and not near the poles where only small amount of solar heating comes.

We expect the strong storms that develop along the hot equator and extract energy from the hot water to be more powerful and abundant when the ocean is hotter. The map however, does not show a hotter equator.

\section{|885-|894}

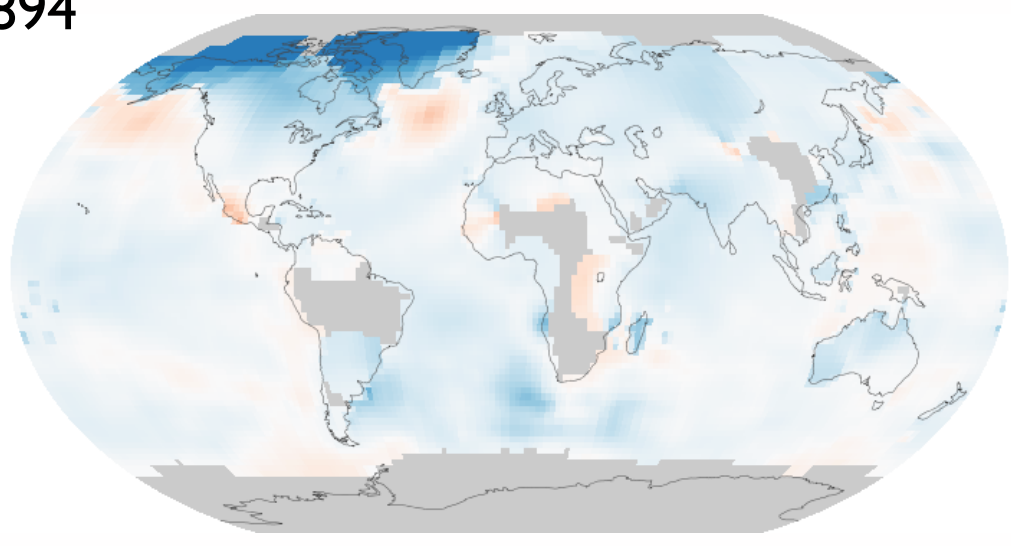

Figure 8: The temperature anomaly for the decade 1885-1894. Note that there are no measurements over vast regions of land. 


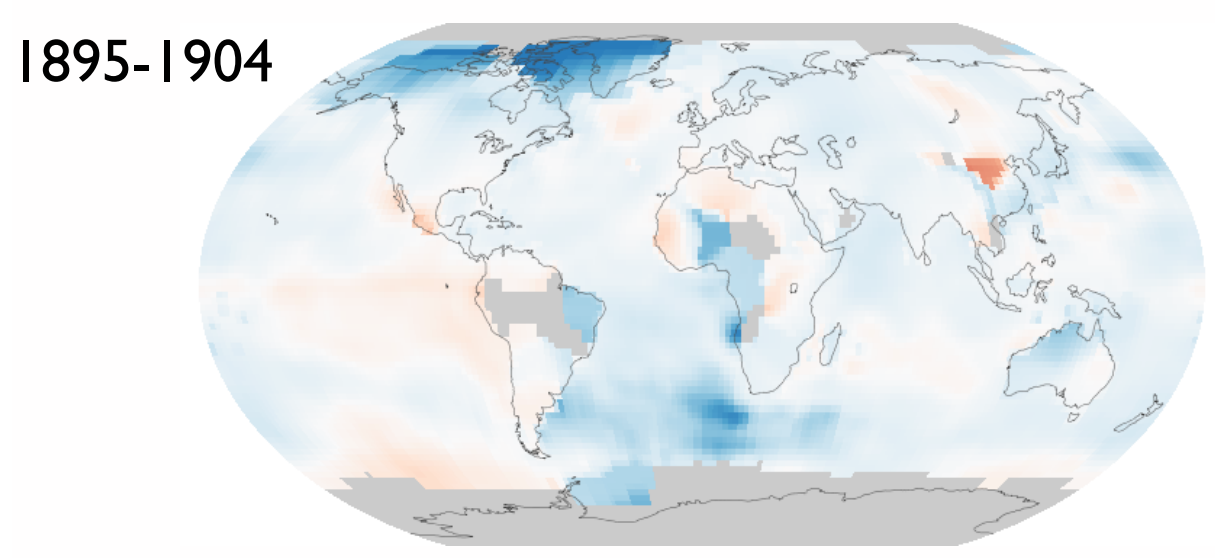

Figure 9: The temperature anomaly for the decade 1895-1904.

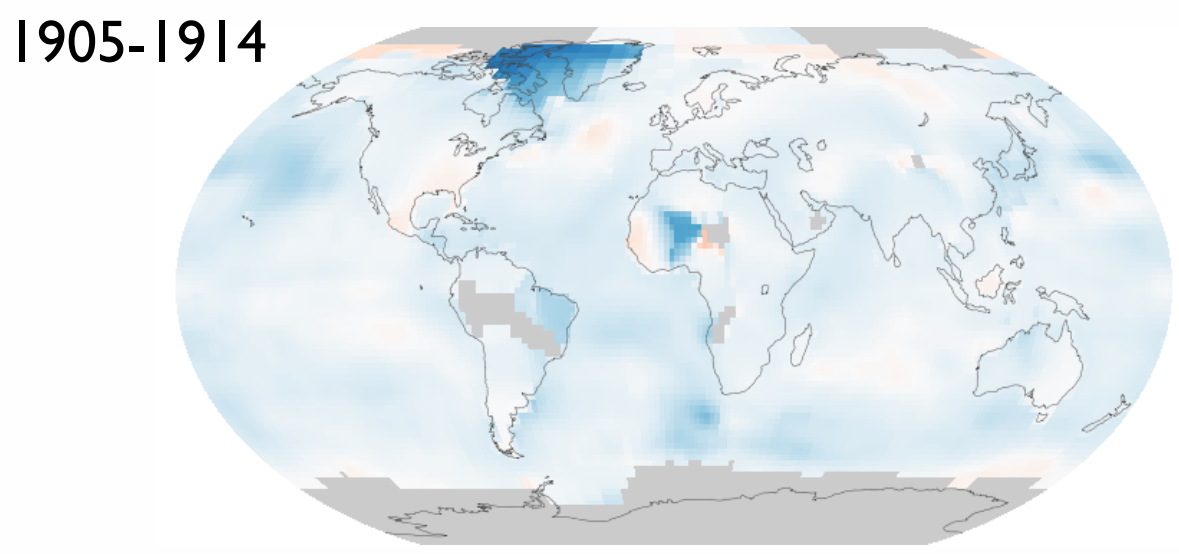

Figure 10: The temperature anomaly for the decade 1905 -1914. Antarctica has no weather station. The oceans show differences tough the measuring is very sparse. 


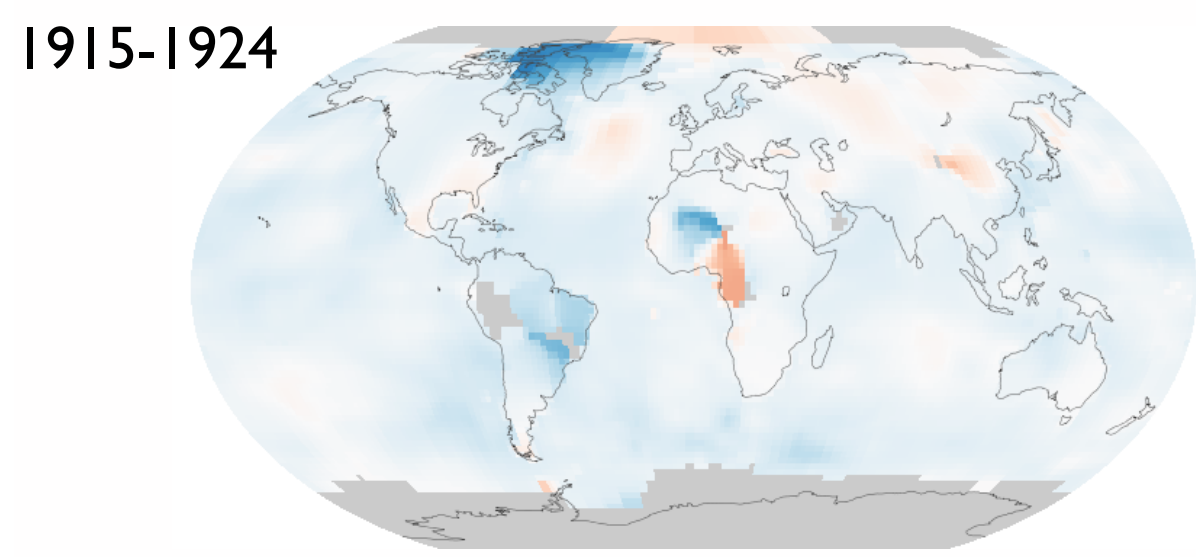

Figure 11: The temperature anomaly for the decade 1915 -1924.

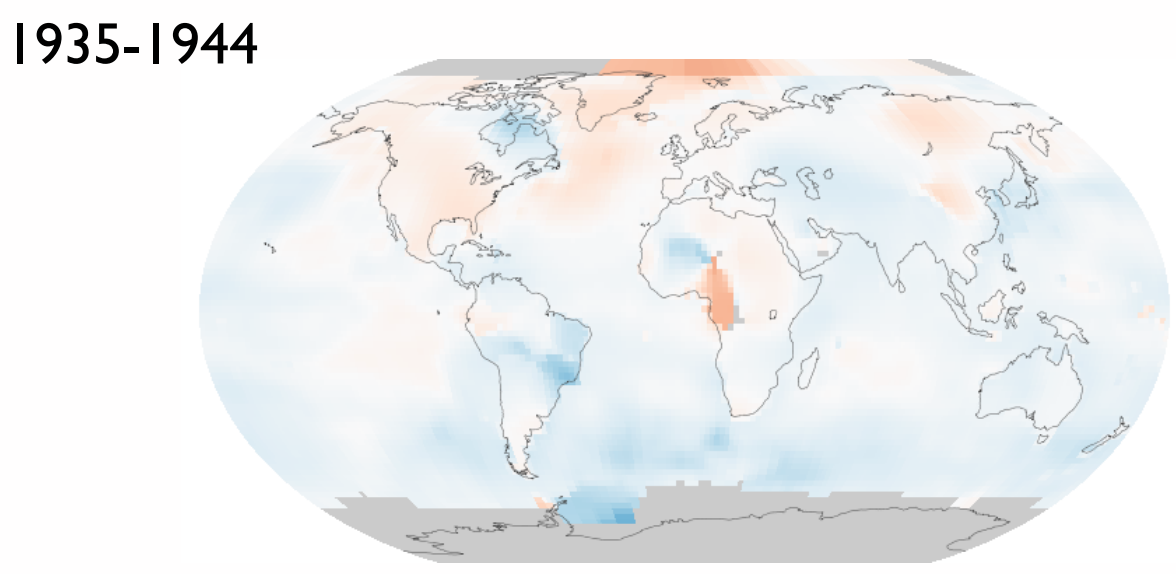

Figure 12: The temperature anomaly for the decade $1935-1944$. 


\section{|945-1954}

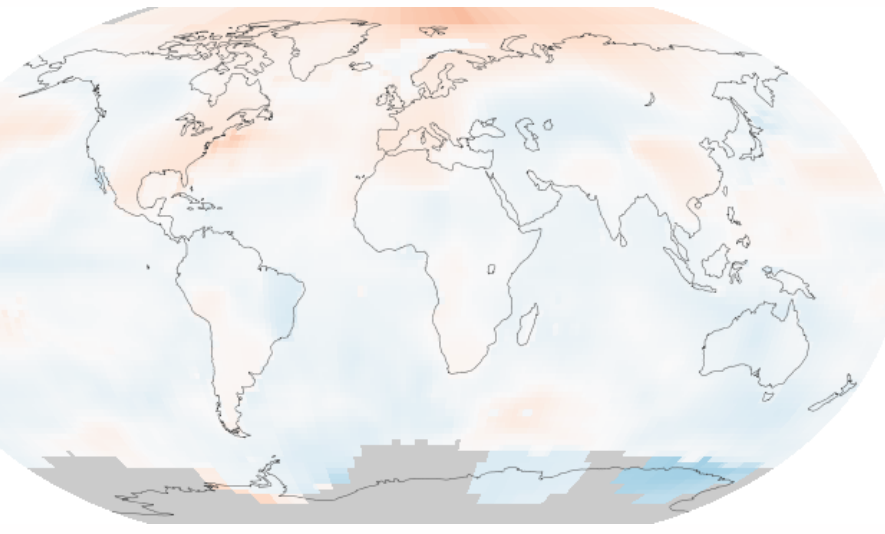

Figure 13: The temperature anomaly for the decade 1945 -1954.

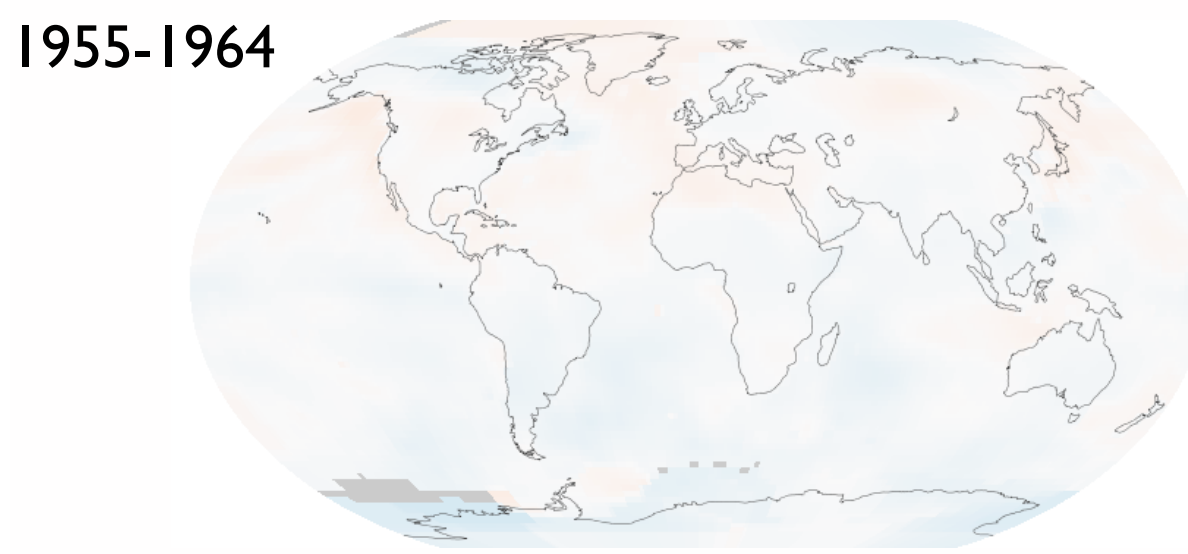

Figure 14: The temperature anomaly for the decade 1955 -1964. 


\section{$1975-1984$}

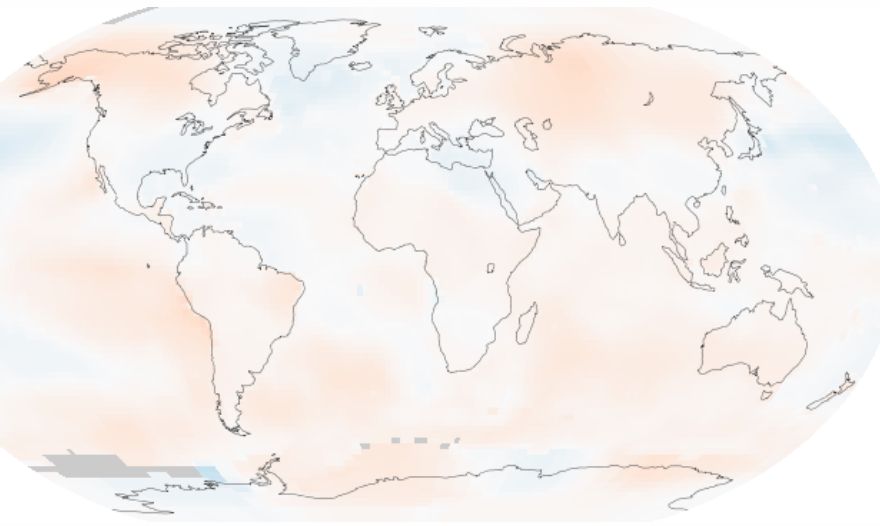

Figure 15: The temperature anomaly for the decade 1975 -1984.

\section{$1985-1994$}

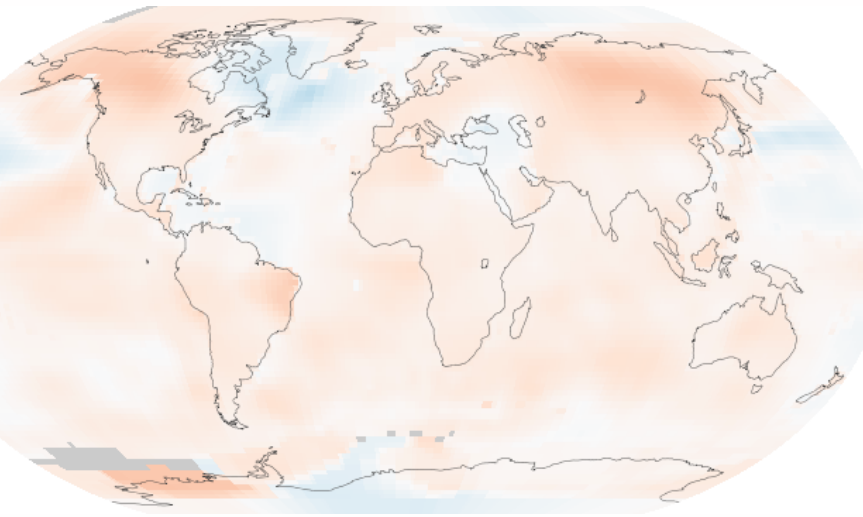

Figure 16: The temperature anomaly for the decade $1985-1994$. 


\section{$1995-2004$}

Figure 17: The temperature anomaly for the decade 1995 -2004. A sudden heating in the North pole appears.

\section{$2005-2014$}
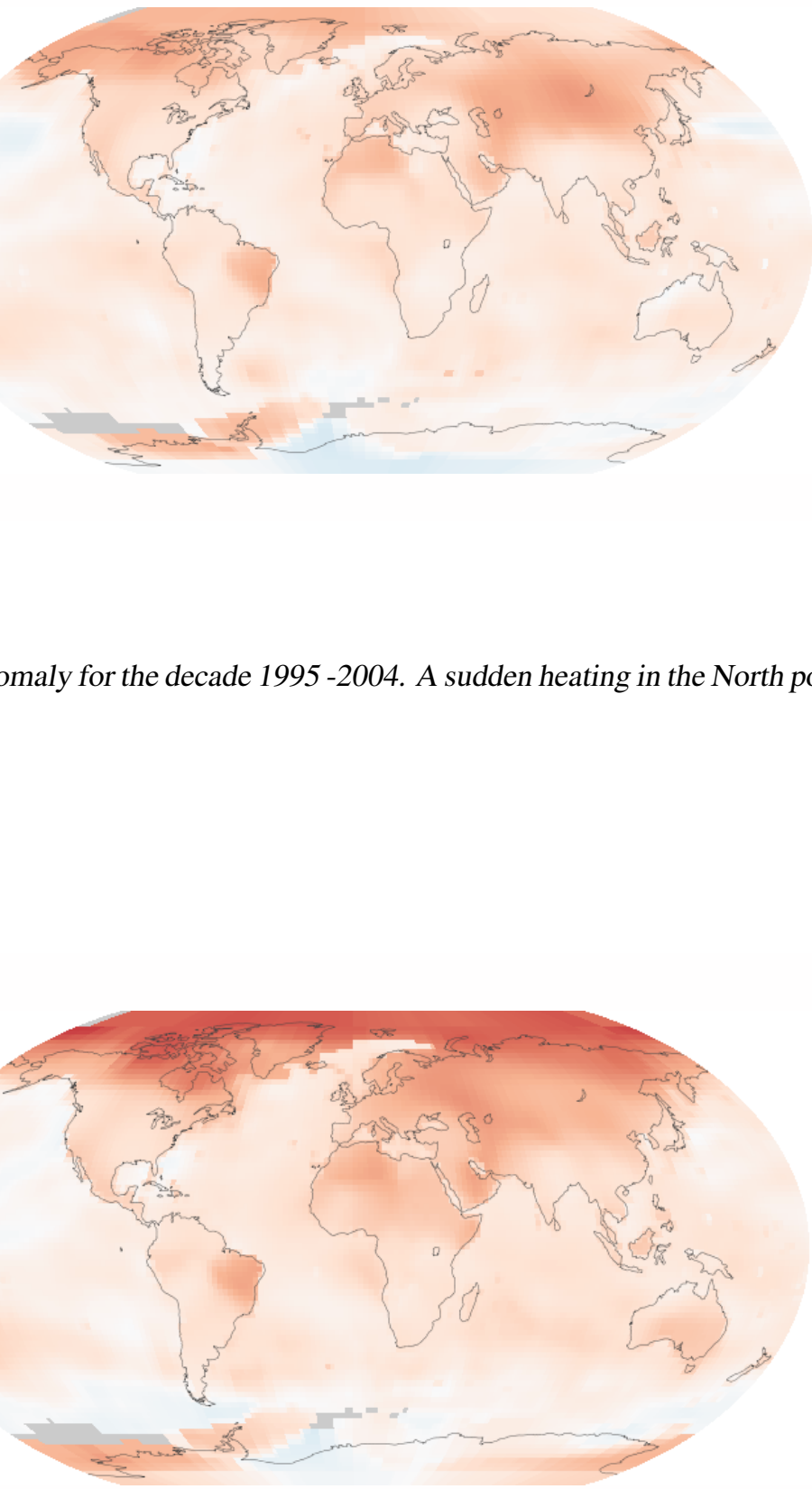

Figure 18: The temperature anomaly for the decade 2005 -2014. 


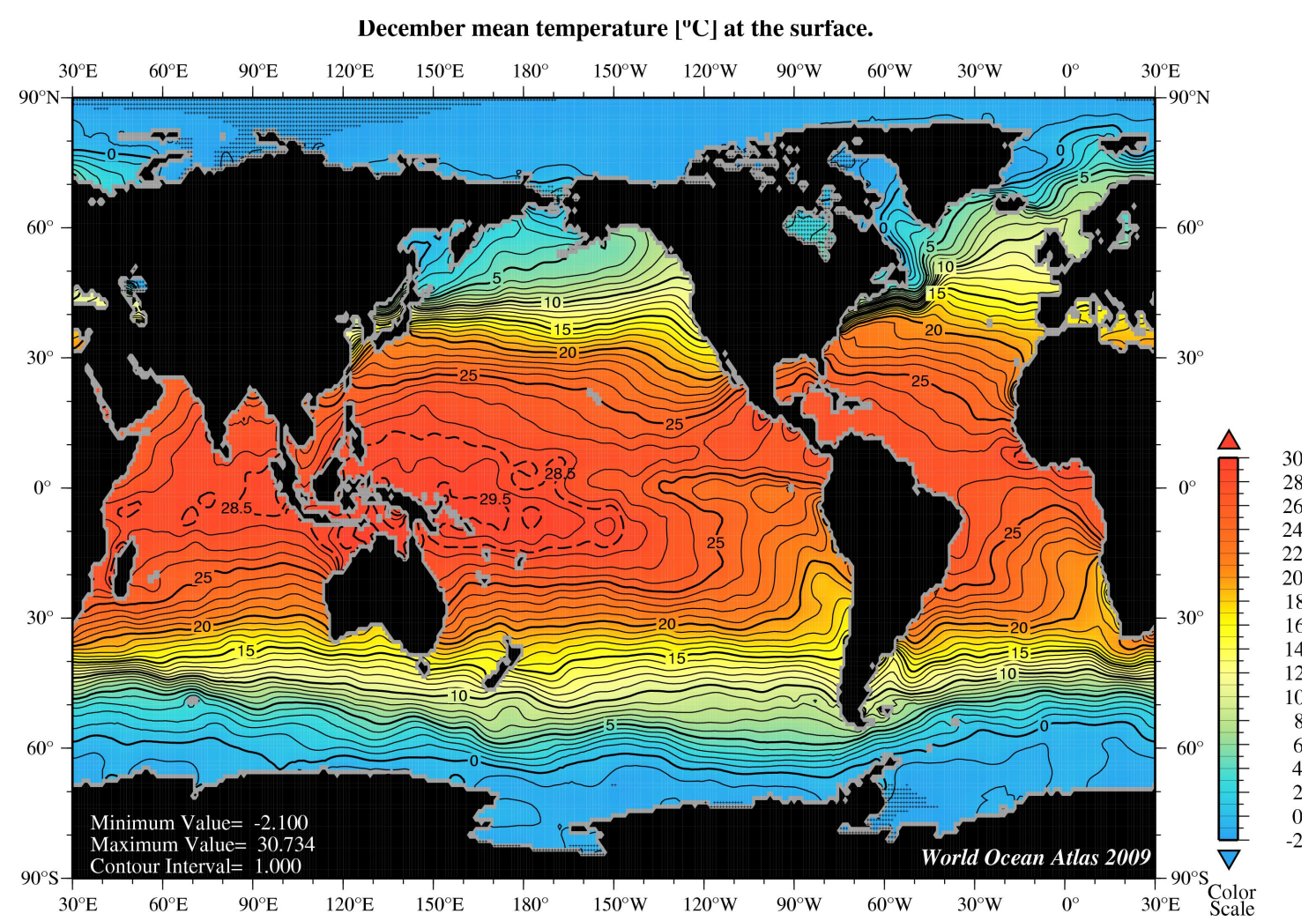

Figure 19: The December mean temperature of the sea surface.

The sea surface temperature is shown in fig. 19. We see the expected change in the temperature and consequently in the specific density. Add to this the variation in local gravity and you infer that there must be circulation which mixes the water and the heating is not local. Fig. 20 is the same as the previous one but at a depth of 100 meter. The comparison between figs. 19, 20 and 21 shows that the heat-wave had hardly time to heat the deeper levels.

Fig. 22 provides the change in temperature anomaly as a function of latitude and time. We see a clear difference between the Northern and the Southern hemispheres. Part of the difference can be attributed to the fact that there is less land and more sea in the Southern hemisphere as well as the fact that the North pole has ice on water while the Southern pole has ice on land.

We see that the years 1980-2000 were cooler than the years 2000-2010. Actually the cold period ended with a significant rise in temperature near the equator which propagated to the North pole but not to the South pole. A similar heating took place close to year 2000.

The comparison between the two hemisphere indicates more activity in the North after year 2000.

\section{The rise of the oceans' level}

Fig. 23 shows the time derivative of the oceans height (in mm/year). This is essentially an expression of the heat capacity times the depth into which the heat penetrated. We find out that 


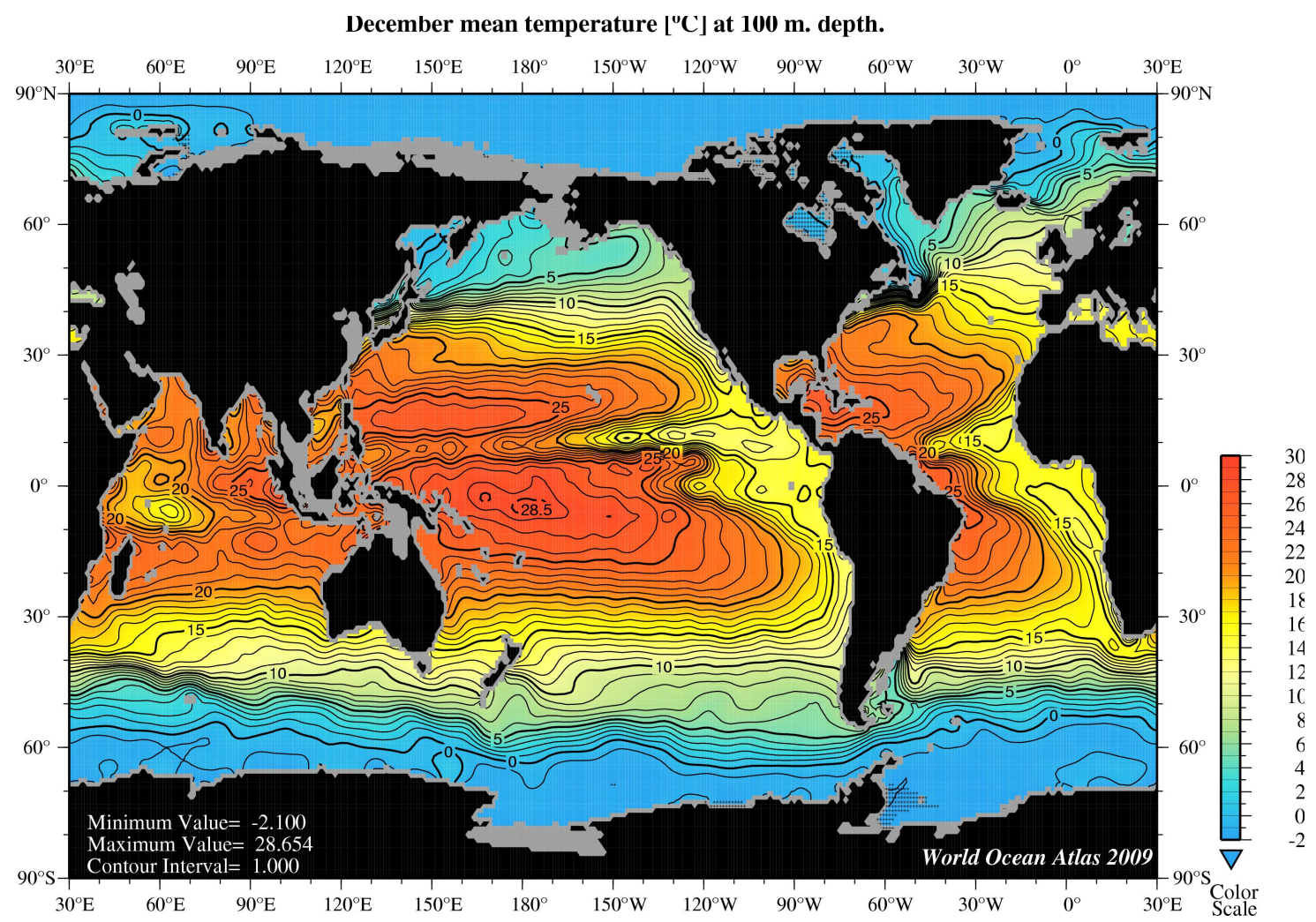

Figure 20: The December mean temperature at a depth of $100 \mathrm{~m}$.

the heat did not penetrate deep, less than few hundred meters. Also note that the derivative is not uniform and the sea rose more in deep regions. Note how non uniforms the measurement in the Pacific ocean.

The figure refers to a short period of time and it is interesting to see what happens on a longer time scale. So in fig 24 we show the change in height since 1950. We see an accelerated rise as of roughly year 2000. However the rise is in millimeters/year. The practically flat measurement between the years 1950-1990 is a surprise. There was no ocean heating?

In fig 25 we find that, as a matter of fact, the sea level oscillates in complete agreement with the solar cycle. The solar wind which is modulated by the number of Sunspots screens the Earth atmosphere from ionizing cosmic rays and these in turn modulate the amount of clouds and hence the albedo $^{12}$.

The situation is however different on geological timescales as seen in fig. 26. We see that the solar induced oscillations have a minute amplitude and cannot be observed on a scale of meters.

Fig 26 shows that the rise in the ocean has started long before humanity started to increase the level of any gas in the atmosphere and what we see today is the leveling off of the time derivative. Moreover, we see in fig. 27, as observed by N. Shaviv, that there is a very good correlation between the Sea Level Change rate and the Solar flux supporting the claim that the oscillations in

\footnotetext{
${ }^{12}$ Shaviv, N.J., International Journal of Modern Physics A, Volume 20, Issue 29, pp. 6662-6665 (2005)
} 


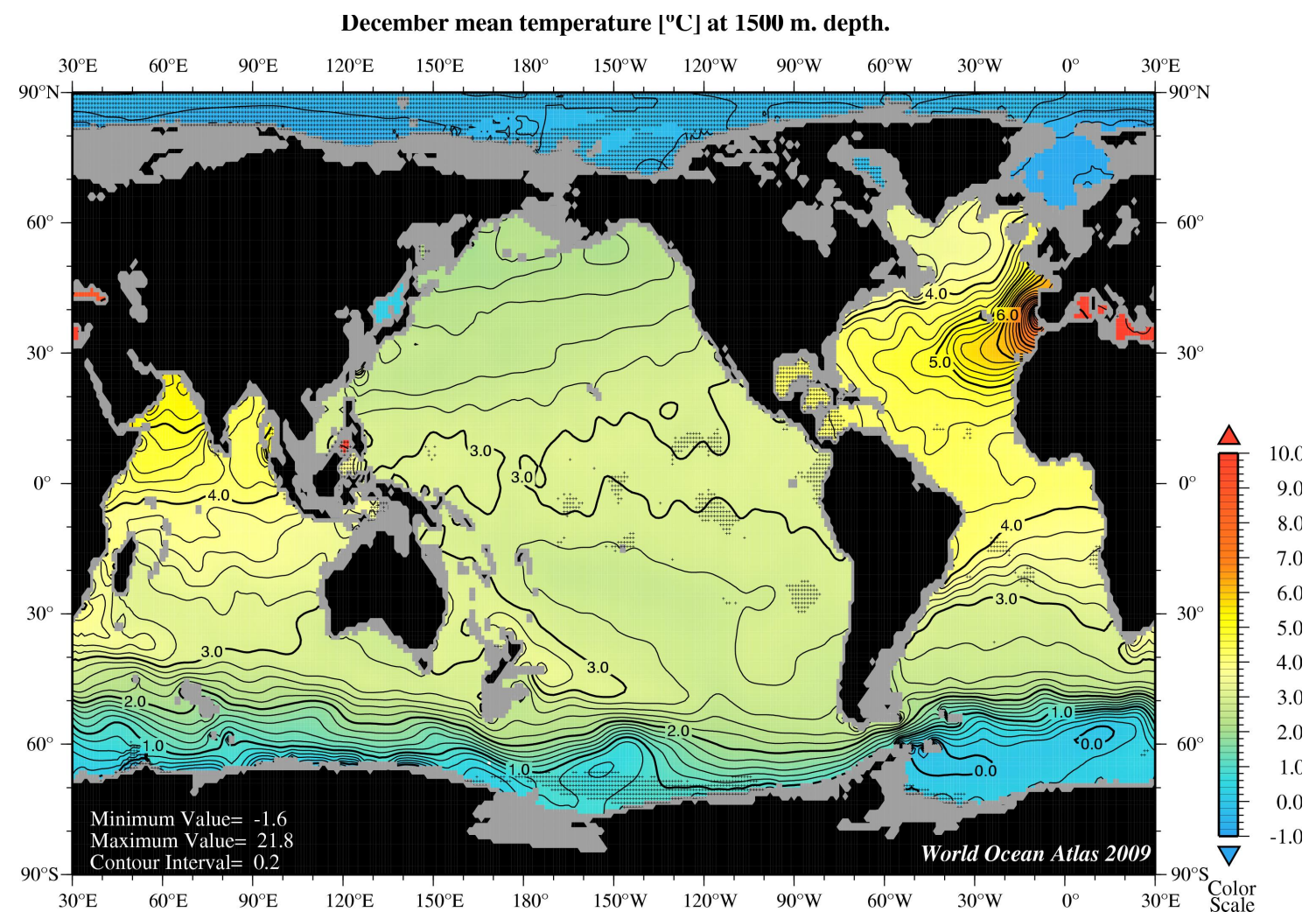

Figure 21: The same as previous figure but at a depth of 1500 meters.

the sea level has probably nothing to do with the temperature of the Earth but with the solar cycle of sunspots.

The predicted rise in sea level if continues, is going to flood many coasts. Fig. 28 shows the predicted flooded areas. Real estate brokers should mark these areas as potentially hazardous and hence cheap.

Incidentally, standard weather station should be at a height of 10 meters above the ground. An observation of the unflooded regions as shown in fig. 28 implies that most of the land is not at sea level and corrections are needed for practically all stations.

\section{The behavior of the $\mathrm{CO}_{2}$}

Fig. 29 provides the column density of $\mathrm{CO}_{2}$ as measured from satellites. The $\mathrm{CO}_{2}$ is the heaviest gas in the atmosphere and in principle should concentrate close to the ground. Winds and air turbulence mix the atmosphere quite well but not fully and traces of the sources can be seen on this map. Still the map is not devoid of mysteries. The USA and Western Europe are the major sources of $\mathrm{CO}_{2}$. The rotation of the Earth causes a drift of the $\mathrm{CO}_{2}$ towards the East. This is seen near the East coast of the USA and East Europe but not on the West coast of the USA and 


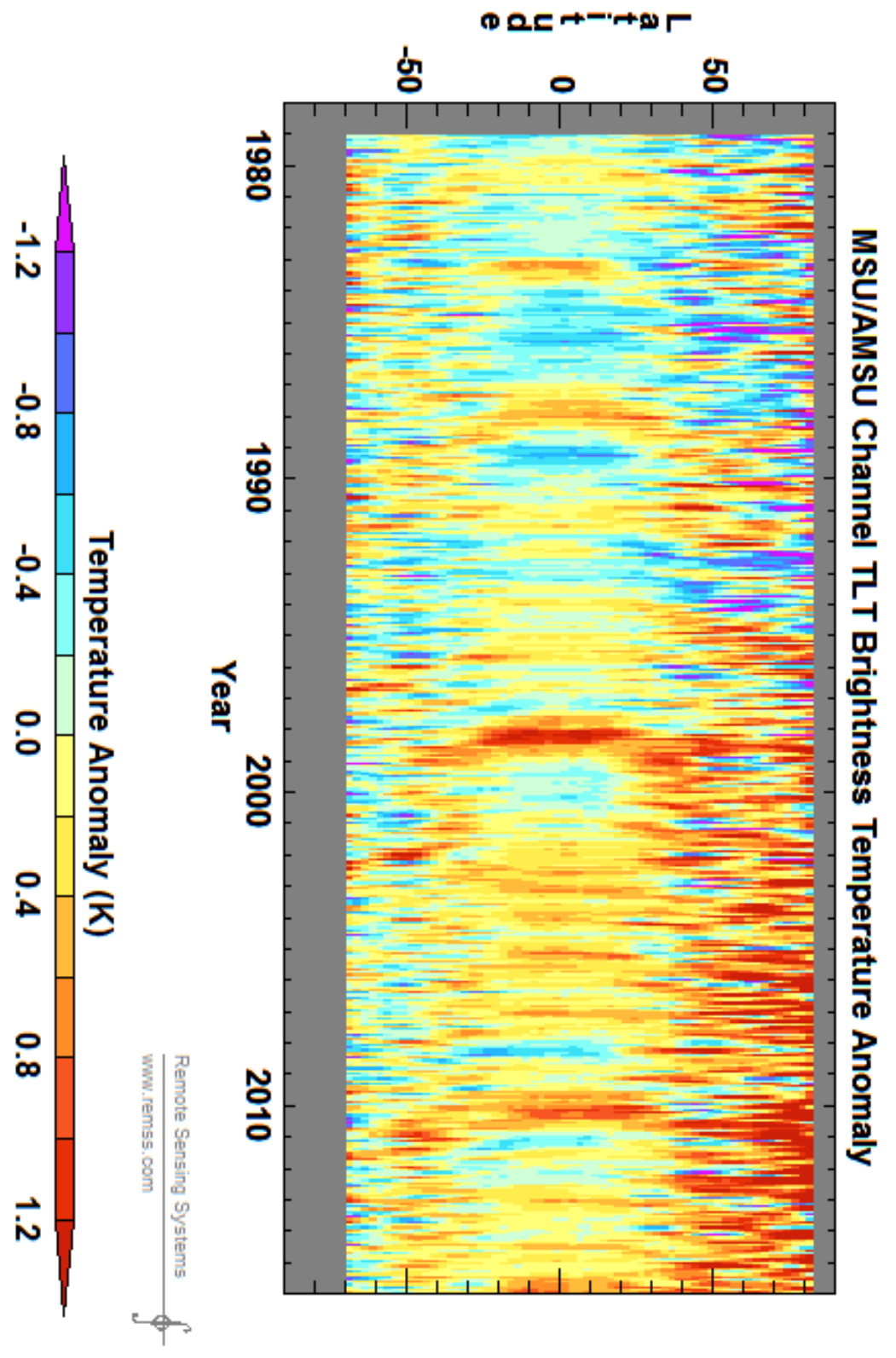

Figure 22: The temperature anomaly as a function of time and latitude. 


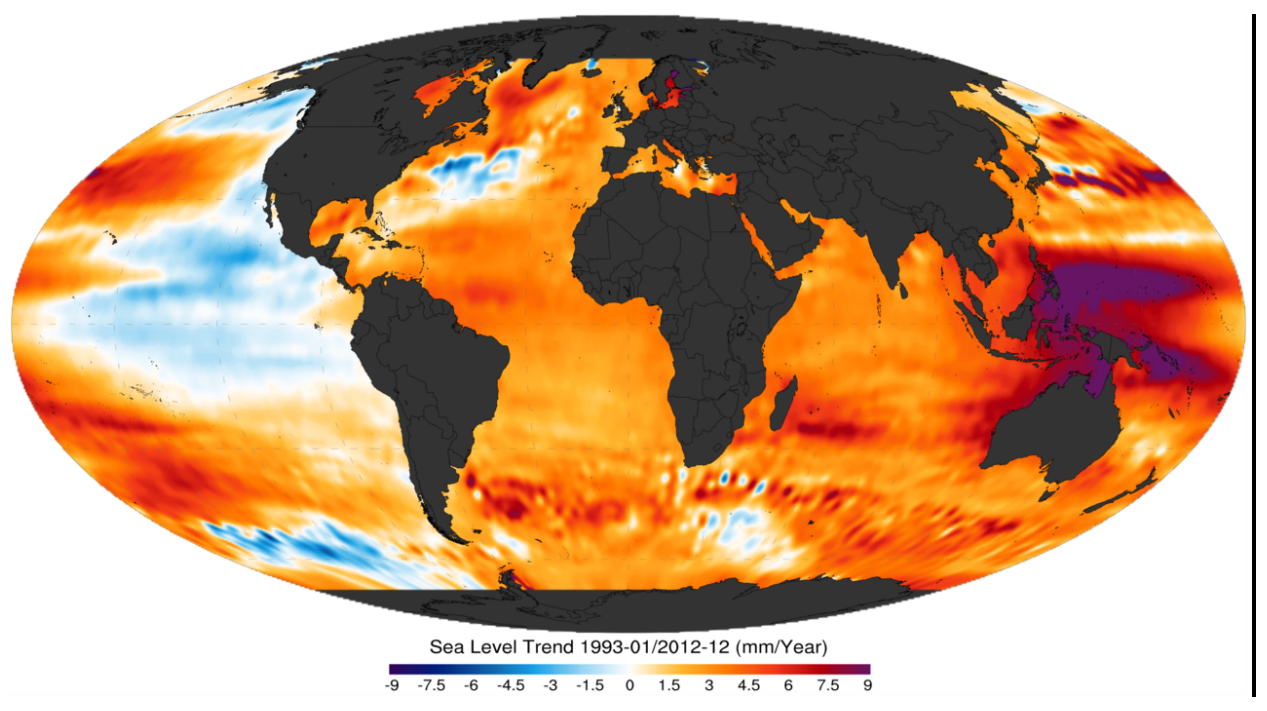

Figure 23: The rate of rise in sea level (in mm/year) during the period 1993-2012.

Argentina. Are local winds more powerful than the rotation of the Earth. In a strict steady state we should see the emitters of $\mathrm{CO}_{2}$.

Note that the heating in recent years took place in the Northern pole where we observe a paucity of $\mathrm{CO}_{2}$ and there was practically no heating where the concentration of $\mathrm{CO}_{2}$ increased. The two poles have lower than average concentration of $\mathrm{CO}_{2}$ but one pole heats while the other cools cf. fig. 1.

If you plot the $\mathrm{CO}_{2}$ on a time scale of 400,000yrs you find the result shown in fig. $30^{13}$. We see that prehistoric $\mathrm{CO}_{2}$ concentration varies periodically but the peak never exceeded about 300ppm. Present day $\mathrm{CO}_{2}$ concentration has already reached 400ppm and the question raised by the IPCC is whether this rise is responsible for the claimed rise in Global Temperature? The answer of the IPCC is in the affirmative! The logic: since both $\mathrm{r} \mathrm{CO}_{2}$ and rise, it is the first which affects the second.

We show in fig. 31 the behavior of methane during the past 1000 years and we see a clear rise. So should we claim that the rise in $\mathrm{CO}_{2}$ drove the temperature and methane concentration $\left(\mathrm{CH}_{4}\right)$ up or was it the rise in temperature which drove the concentration of all gases up? We reject the possibility that the $\mathrm{CO}_{2}$ affected the temperature and as a consequence the temperature affected the methane. Recall, the methane is a significantly more powerful greenhouse gas than $\mathrm{CO}_{2}$ and the argument of the IPCC should have been based on methane. Also, the methane concentration changed with geological times exactly like the $\mathrm{CO}_{2}$ namely it has peaks and minima. Needless to point out that $\mathrm{N}_{2} \mathrm{O}$ shows an exactly similar behavior.

We face the $\mathrm{CO}_{2}$ paradox: $98 \%$ of the $\mathrm{CO}_{2}$ on Earth is dissolved in the ocean where the concentration is sensitive to temperature. Suppose the $\mathrm{CO}_{2}$ has a strong effect on the temperature and is the only agent affecting it. Then as the concentration of $\mathrm{CO}_{2}$ in the atmosphere rises it causes the temperature of the atmosphere to rise as well. The $\mathrm{CO}_{2}$ in the atmosphere was in equilibrium

\footnotetext{
${ }^{13}$ The figure mixes two values, the local concentration at the location where the ice core was extracted, with measurement in Hawaii, where the concentration of $\mathrm{CO}_{2}$ in the atmosphere is measured.
} 


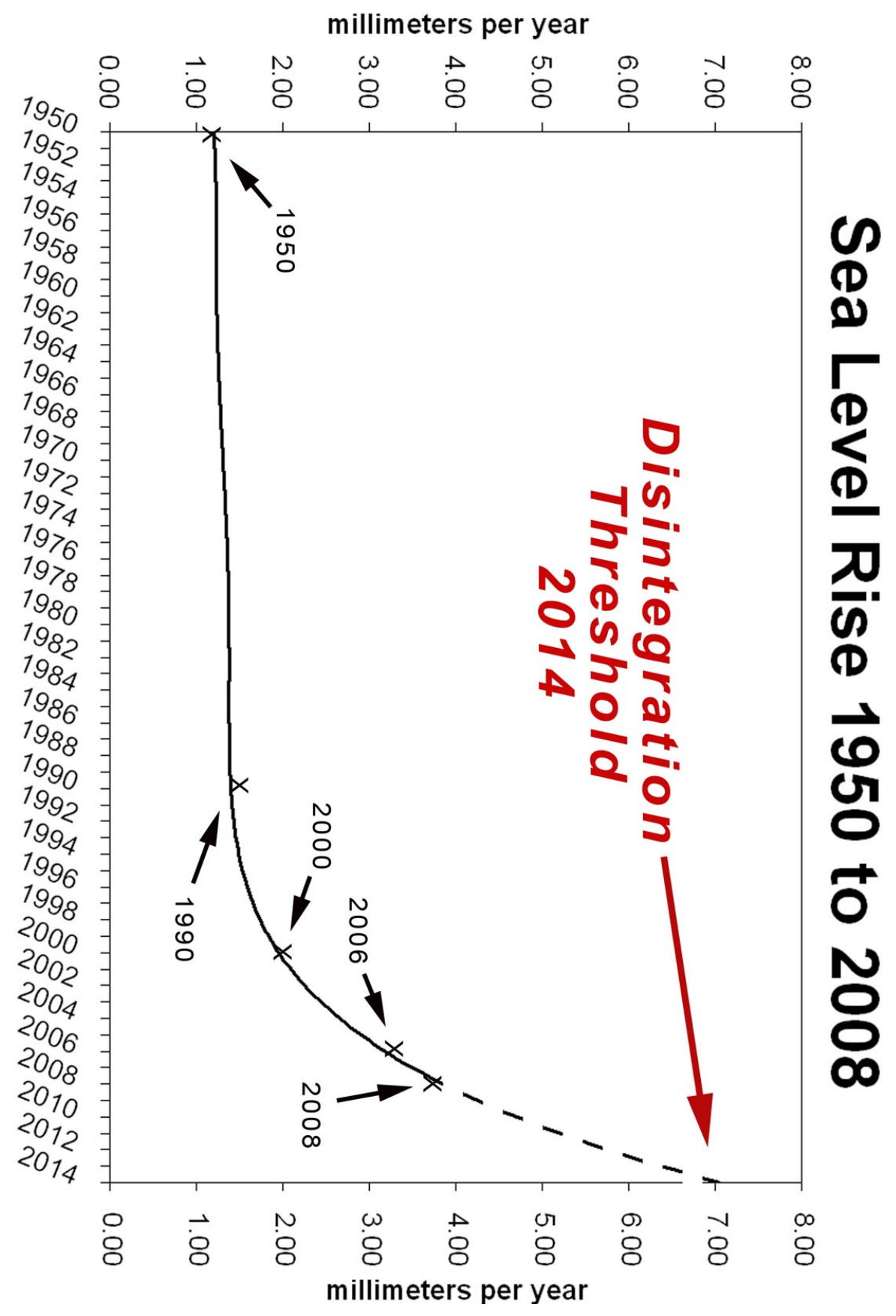

Figure 24: The rate of sea level rise (in mm/year) since 1950. 


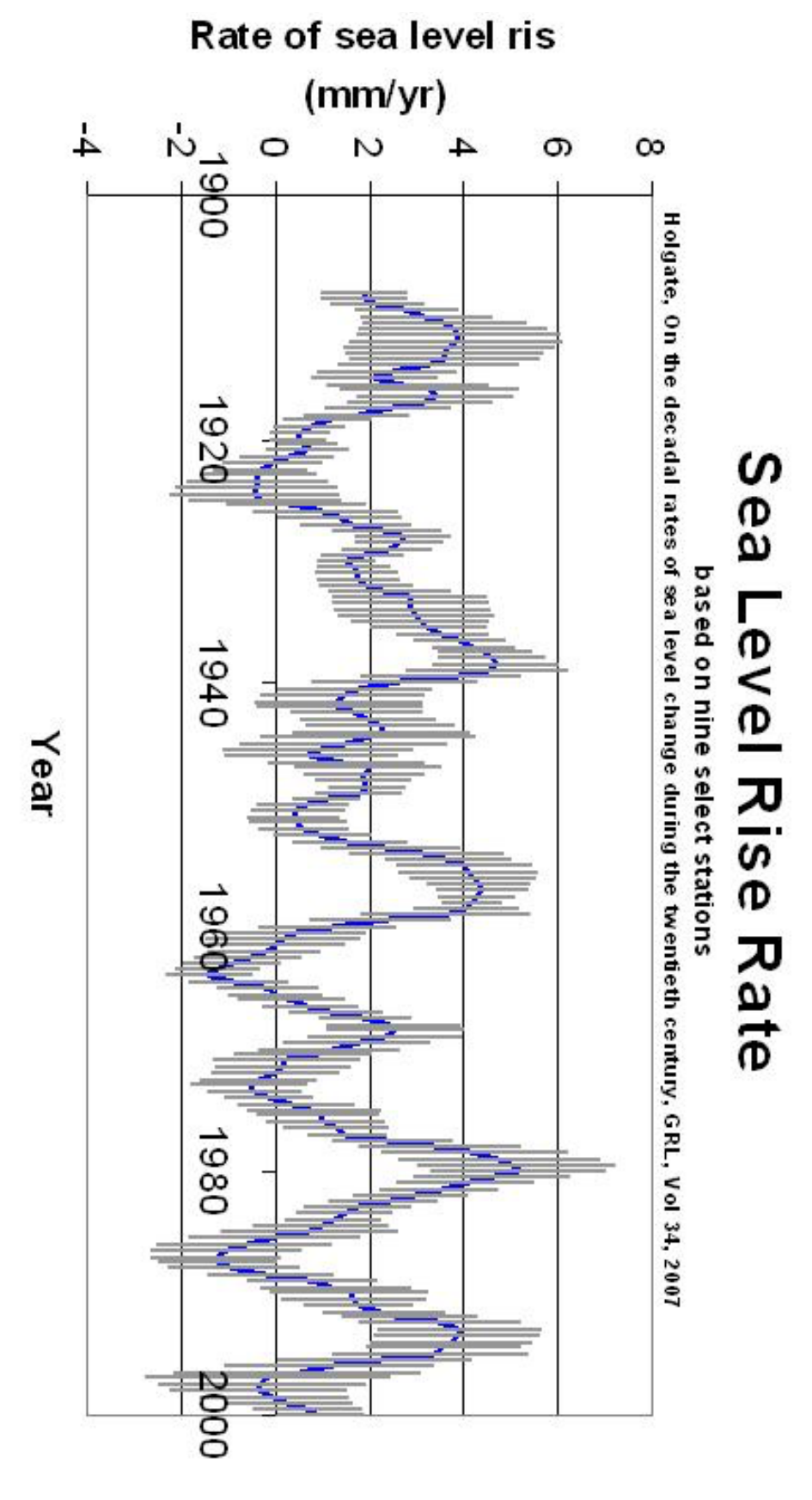

Figure 25: Fine details of the rate of sea level rise (in mm/year) since 1900. Source: Shaviv, N.J. 'Using the oceans as a calorimeter to quantify the solar radiative forcing', Journal of Physical Research, Space Physics, November 2008. 


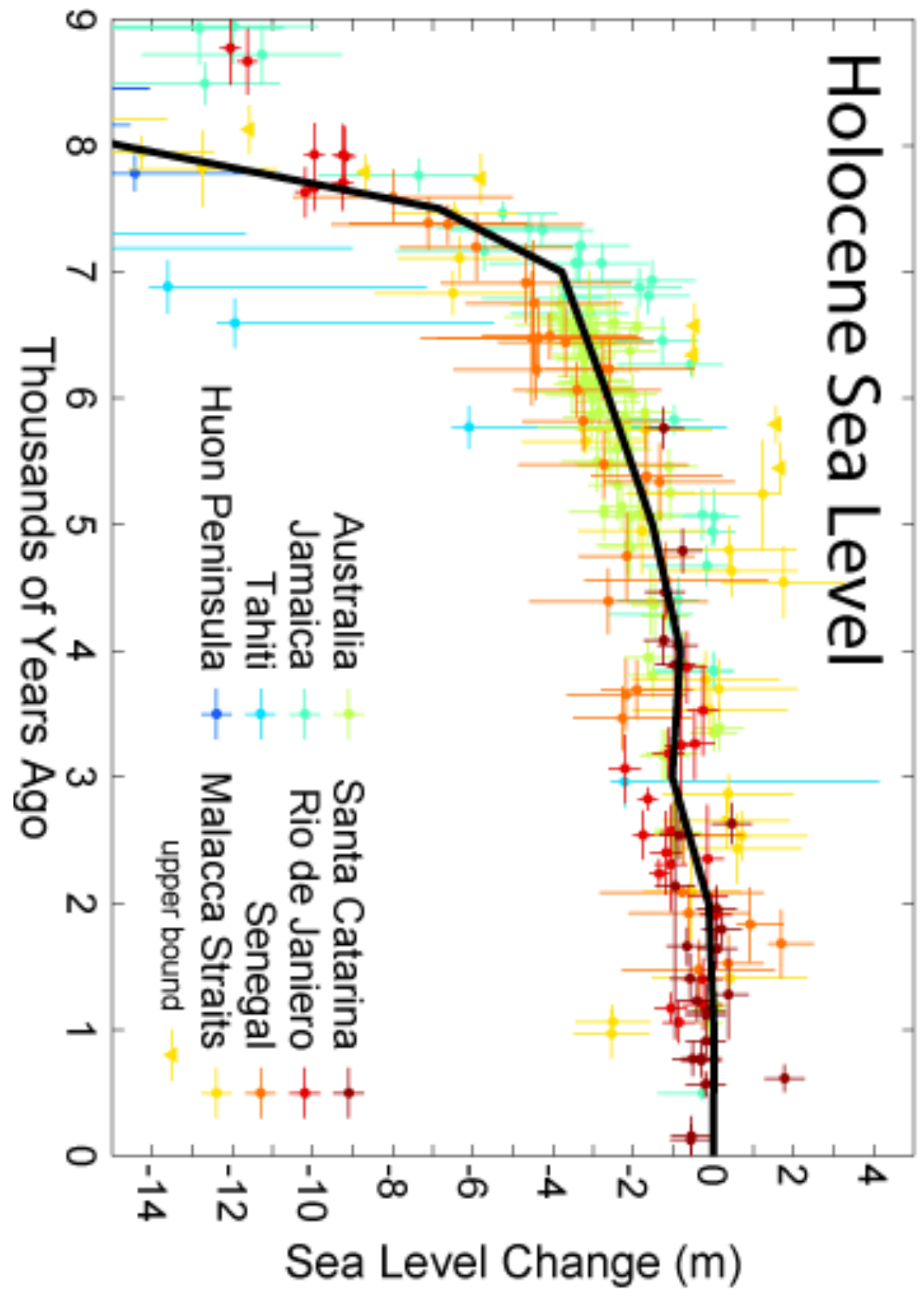

Figure 26: Fine details of the sea level (in meters) since geological times. 


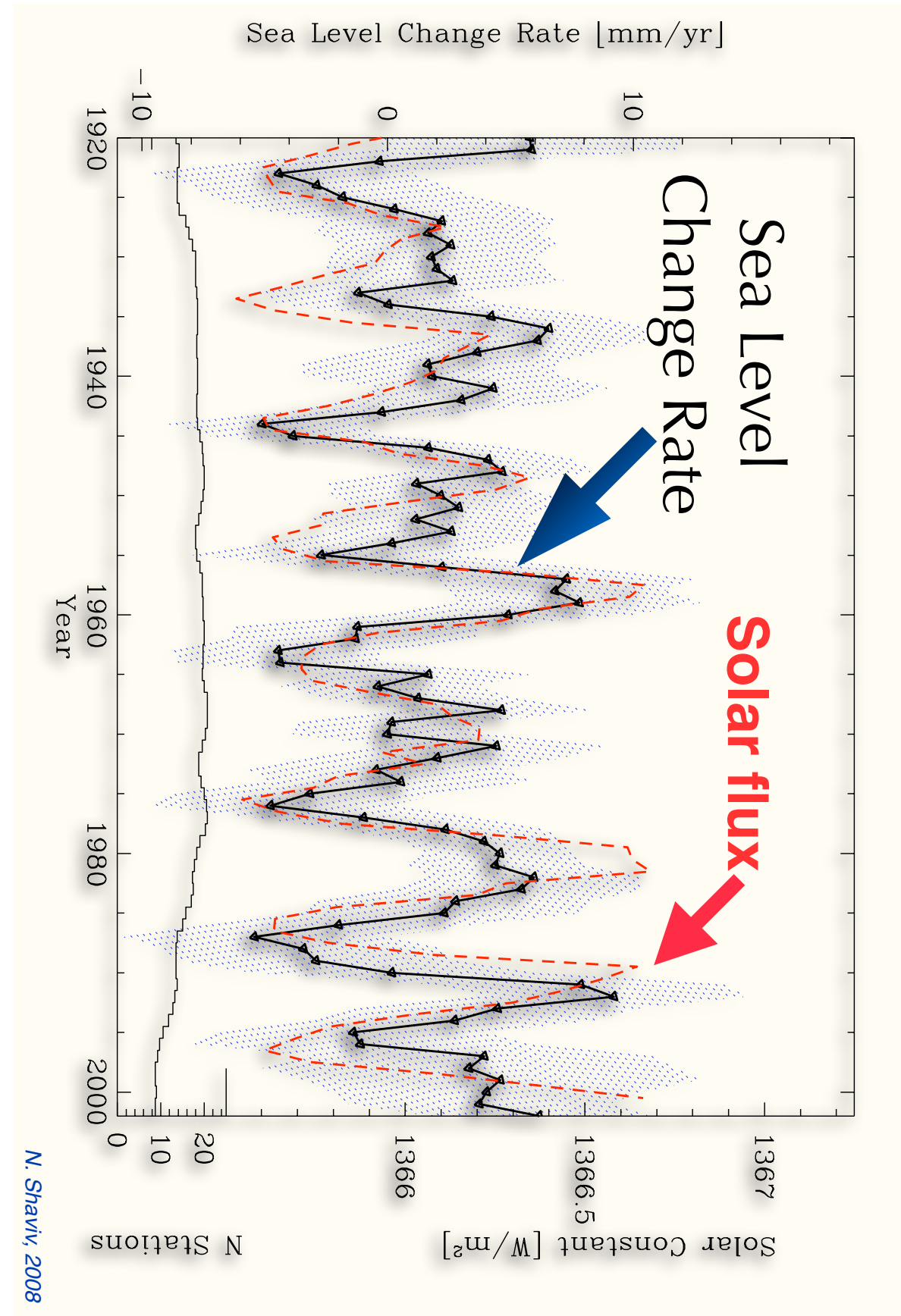

Figure 27: The correlation between sea level change and solar flux. 


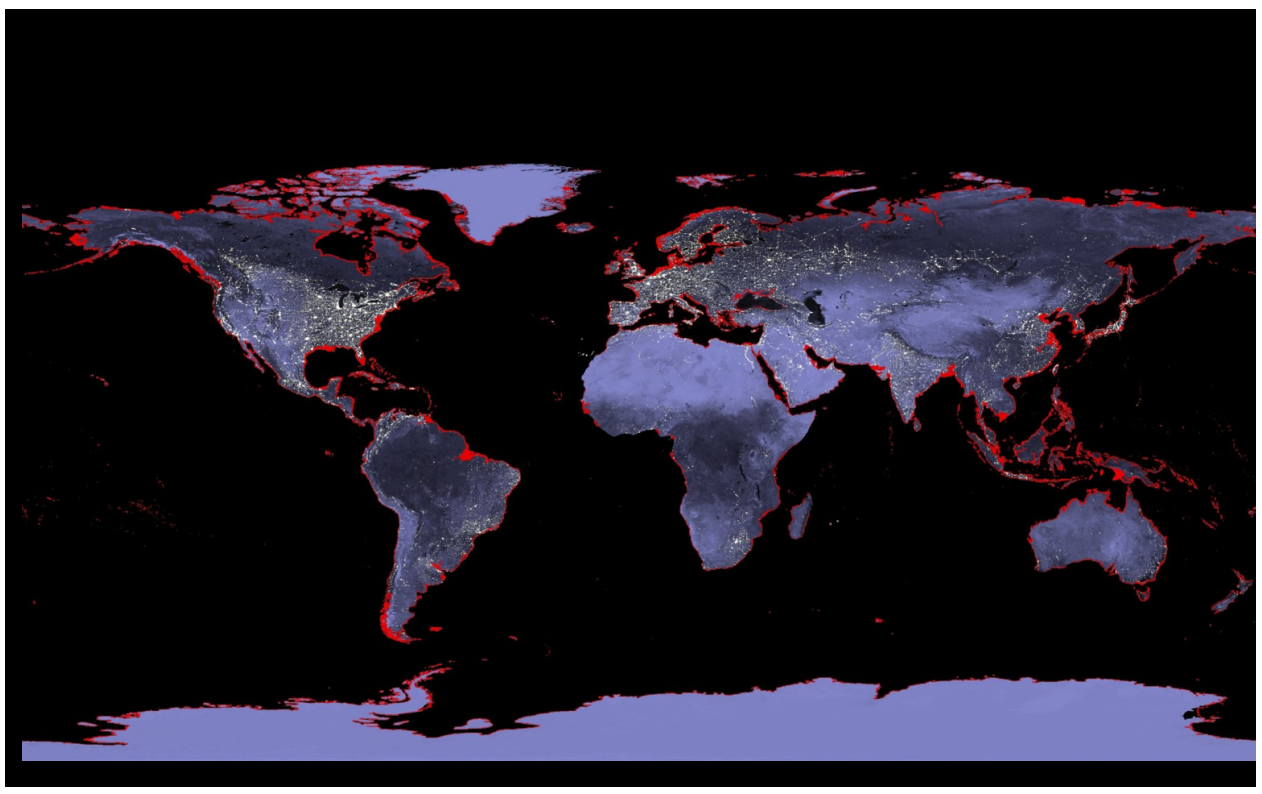

Figure 28: The effect of a 6 meter rise in sea level. Flooded areas are red colored.

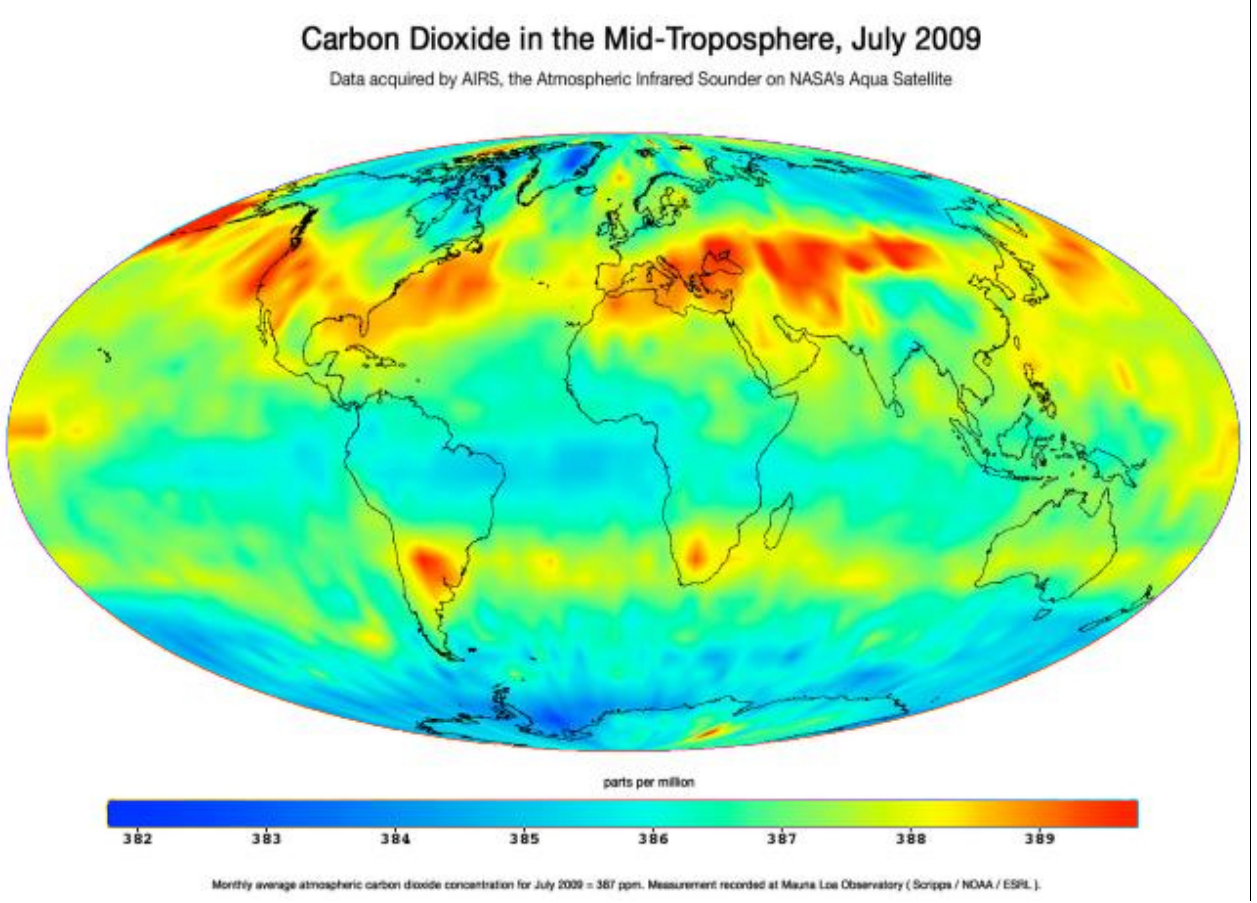

Figure 29: The concentration of $\mathrm{CO}_{2}$ in the mid-troposphere 


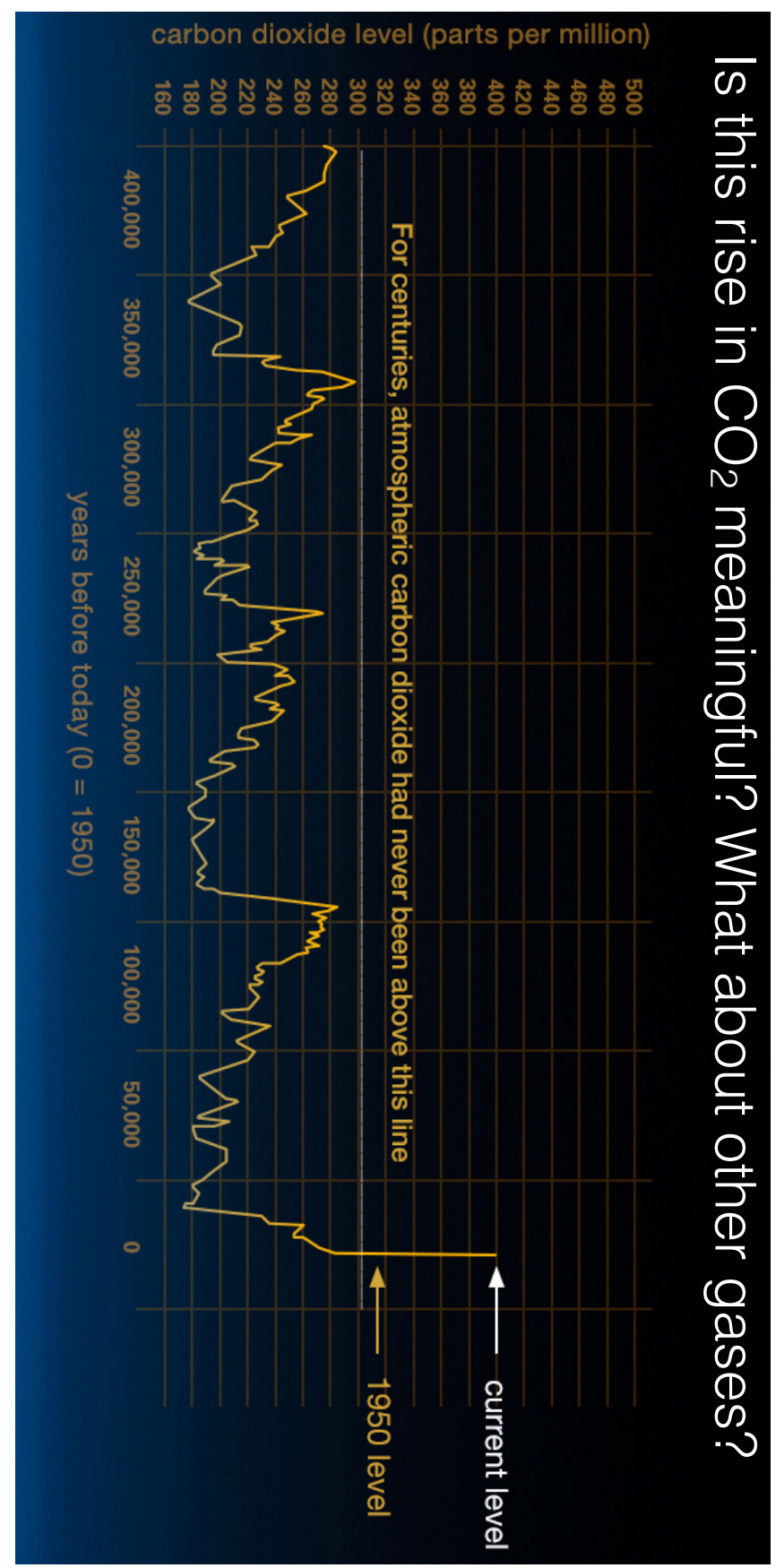

Figure 30: The concentration of $\mathrm{CO}_{2}$ as a function of time since about 400,000 yrs ago. The question is to what extent does the recent rise of $\mathrm{CO}_{2}$ imply that it causes the temperature rise? 


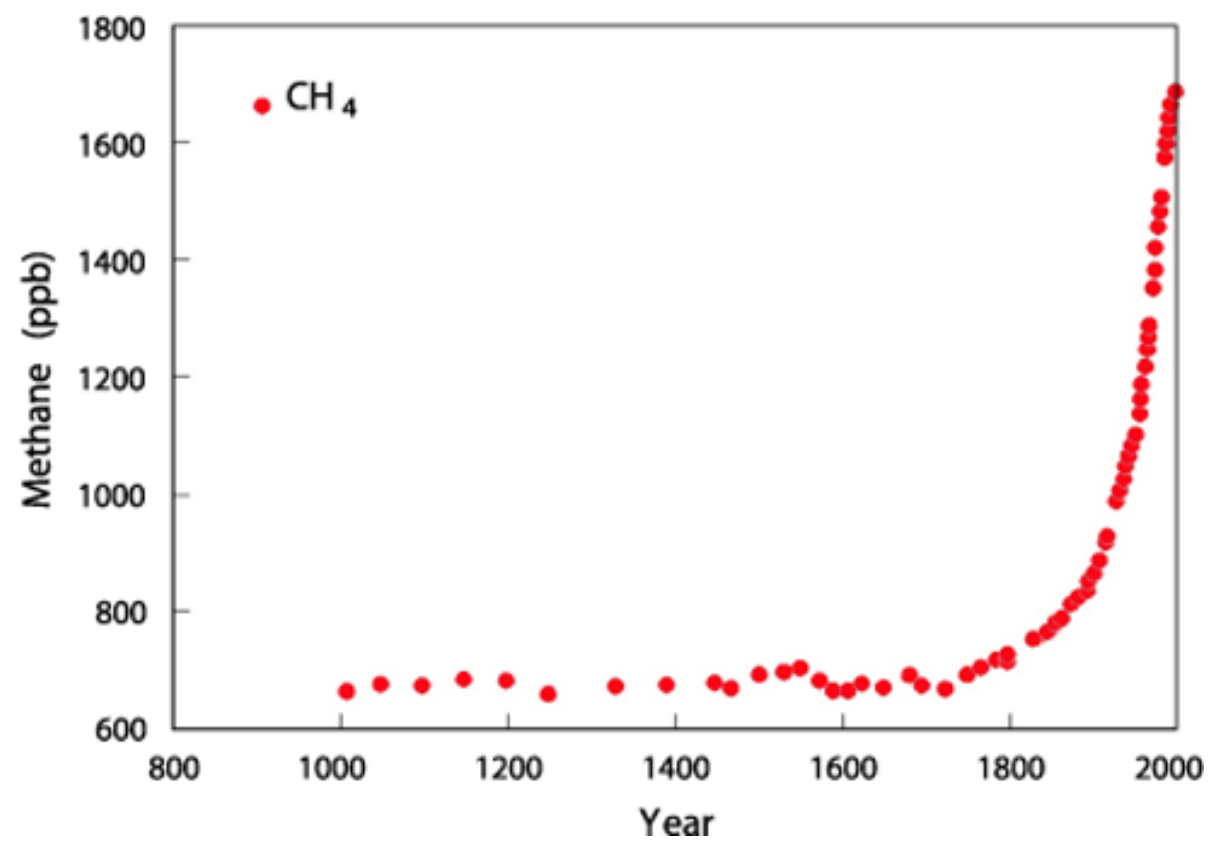

Source: CSIRO Atmospheric Research

Figure 31: The concentration of Methane in the atmosphere over the past $1000 \mathrm{yrs}$.

with a sea-concentration proper for the temperature. As the concentration of $\mathrm{CO}_{2}$ increases in the atmosphere it raises the air temperature. As a consequence, more $\mathrm{CO}_{2}$ is extracted from the ocean into the atmosphere leading to an additional increase of the temperature and causing eventually a run away, in other words, the assumption that $\mathrm{CO}_{2}$ heats the atmosphere above a gigantic storage of $\mathrm{CO}_{2}$ creates a positive feedback which should lead to a runaway. The heating of the atmosphere should occur instantly while the heating of the oceans takes time because of the heat capacity of the oceans. The delay of the heating of the oceans does not change the conclusion that it leads to a run away the times scale of which depends on the heat capacity of the oceans. We however, do not observe on Earth such a runaway (hopefully). There can be several reasons for why such a run away does not show up. If so there must be an additional dominant factor affecting the temperature.

Once the $\mathrm{CO}_{2}$ resides in the atmosphere it can only disappear by being dissolved in the oceans. The time scale for this to happen is about $10^{3}$ years. Hence we should observe that for two decades the amount of $\mathrm{CO}_{2}$ rose from the earlier to the later decade. If the $\mathrm{CO}_{2}$ affects the temperature directly say via the effect on the radiative transfer in the atmosphere, then the later decade with the higher amount of $\mathrm{CO}_{2}$ must be hotter. Thus as the concentration of $\mathrm{CO}_{2}$ always rises, the temperatures can never decrease. But this is not the case, there are rises and decreases in the temperature.

We can see the paradox differently: During the century 1898-1998 the temperature increased according to the IPCC by about $0.72 \mathrm{~K}$ while the $\mathrm{CO}_{2}$ concentration had risen from $295 \mathrm{ppm}$ to $367 \mathrm{ppm}$, namely a rise of $73 \mathrm{ppm}$ per century. 
However, the $\mathrm{CO}_{2}$ has increased from 367ppm to 403ppm since 1998, namely 36ppm while the temperature had been stable.

The $\mathrm{CO}_{2}$ acts on the radiative transfer instantly and there is no time delay in its action of heating. If mysteriously the $\mathrm{CO}_{2}$ does not act right away we have to assume some time scale for the effect. So where does the $\mathrm{CO}_{2}$ accumulate? The absorption in the atmosphere takes place in few bands and has wide windows. The optical depth of the absorption band is high, $10-10^{4}$ so that the absorption is heavily saturated. Consequently the additional absorption of the $\mathrm{CO}_{2}$ takes place high in the atmosphere and has a negligible effect near the ground.

\section{The acidity of the seas}

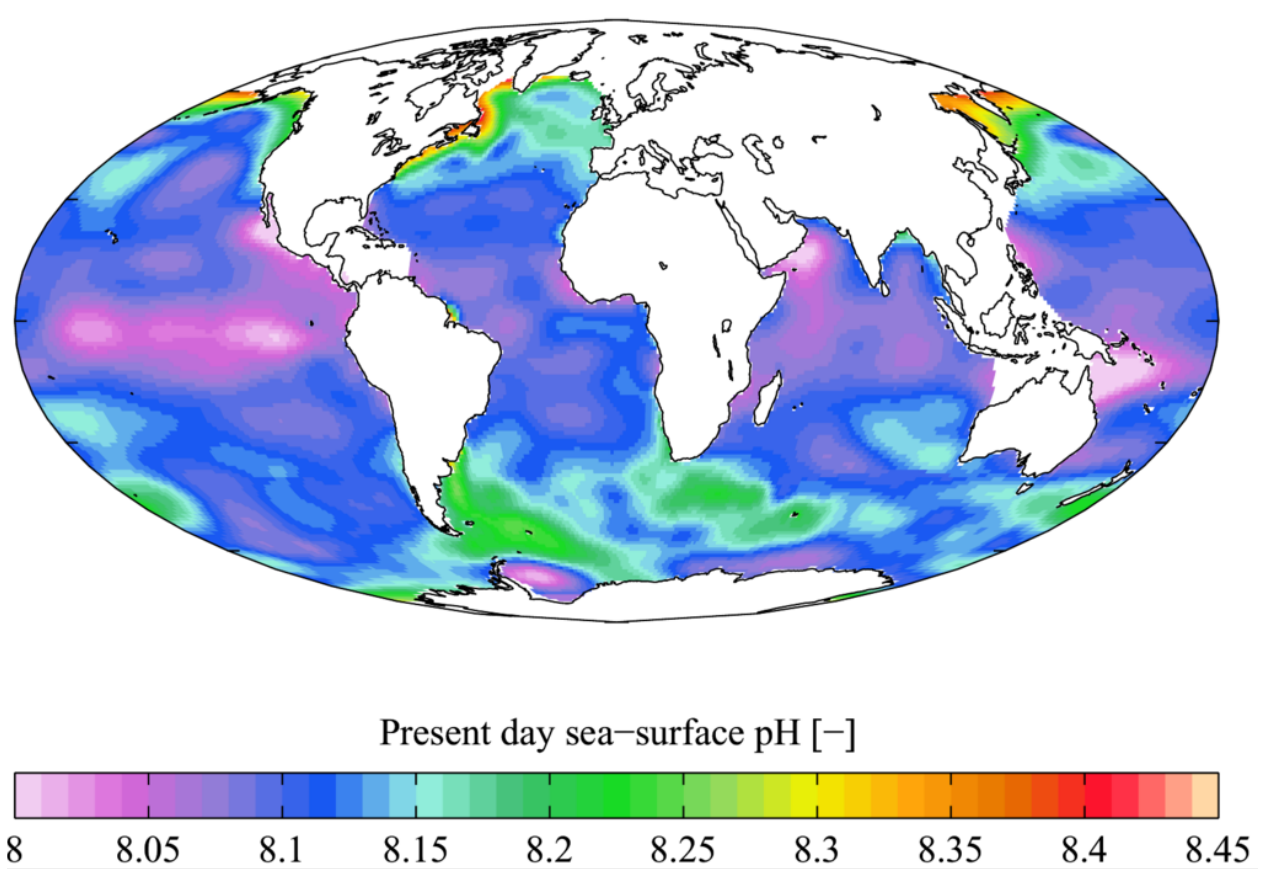

Figure 32: The distribution of sea-surface $p H$.

The distribution of present day $\mathrm{pH}$ in the oceans is shown in fig. 32. The $\mathrm{pH}$ at the beginning of the industrial period was about 8.15. The dissolution of the $\mathrm{CO}_{2}$ in the ocean creates carbonic acid $\left(\mathrm{HCO}_{3}^{-}\right)$which in turn increases the acidity of the water. This change is expected to gradually destroy the ocean life. As a matter of fact, the change of the $\mathrm{pH}$ is the largest threat to mankind and not global warming by few degrees.

The change in the $\mathrm{pH}$ as a function of the concentration of $\mathrm{CO}_{2}$ from 1850 till today is shown in fig. 33. We note the accelerated rate of change due to the increase in the concentration of $\mathrm{CO}_{2}$. The predicted change of the $\mathrm{pH}$ in coming years is shown in fig 34 .

$\mathrm{CO}_{2}$ dissolves very slowly in the oceans. The map of $\mathrm{CO}_{2}$ in the upper part of the seas is shown in fig. 35. We see that $\mathrm{CO}_{2}$ dissolves mainly in the Atlantic ocean and very little in the other oceans. The may be due to underwater volcanos. The effective sea area required to dissolve the gas and reduce it in the lower atmosphere is significantly smaller than the total area of oceans. 


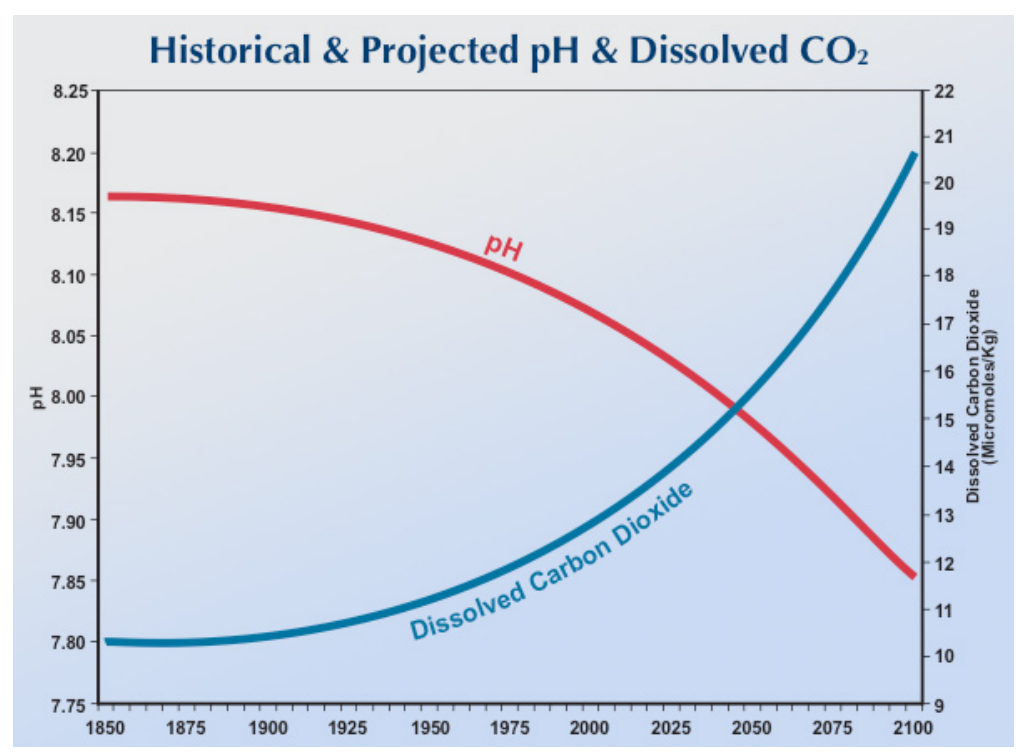

Figure 33: The change in the $\mathrm{pH}$ as a function of the concentration of $\mathrm{CO}_{2}$ from 1850 till today.

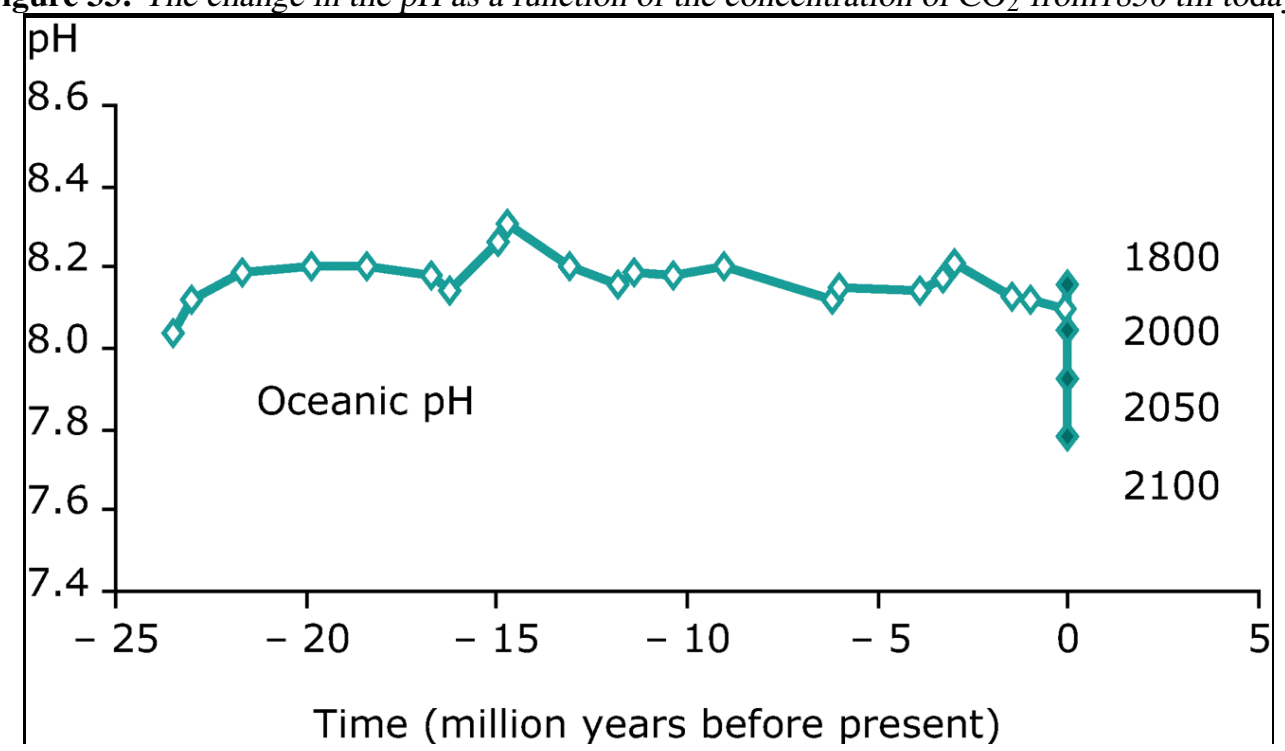

Figure 34: The change in the $\mathrm{pH}$ as a function of the concentration of $\mathrm{CO}_{2}$ from1850 till today.

Fig. 36 shows the effect of the seasons on the $\mathrm{pH}$ along with the gradual decrease.

The column density of $\mathrm{CO}_{2}$ in the ocean, probably most of the emitted $\mathrm{CO}_{2}$ since the beginning of the industrial revolution (measured with ${ }^{13} \mathrm{C}$ ) is shown in fig. 38.

\section{Underwater Volcanos}

In fig. 39 we see the action of an underwater volcano created at the break between tectonic plates. One can assume that such an activity takes place from time to time along about $40,000 \mathrm{~km}$ of breaks between tectonic plates. We still do not know how often such an activity takes place and 


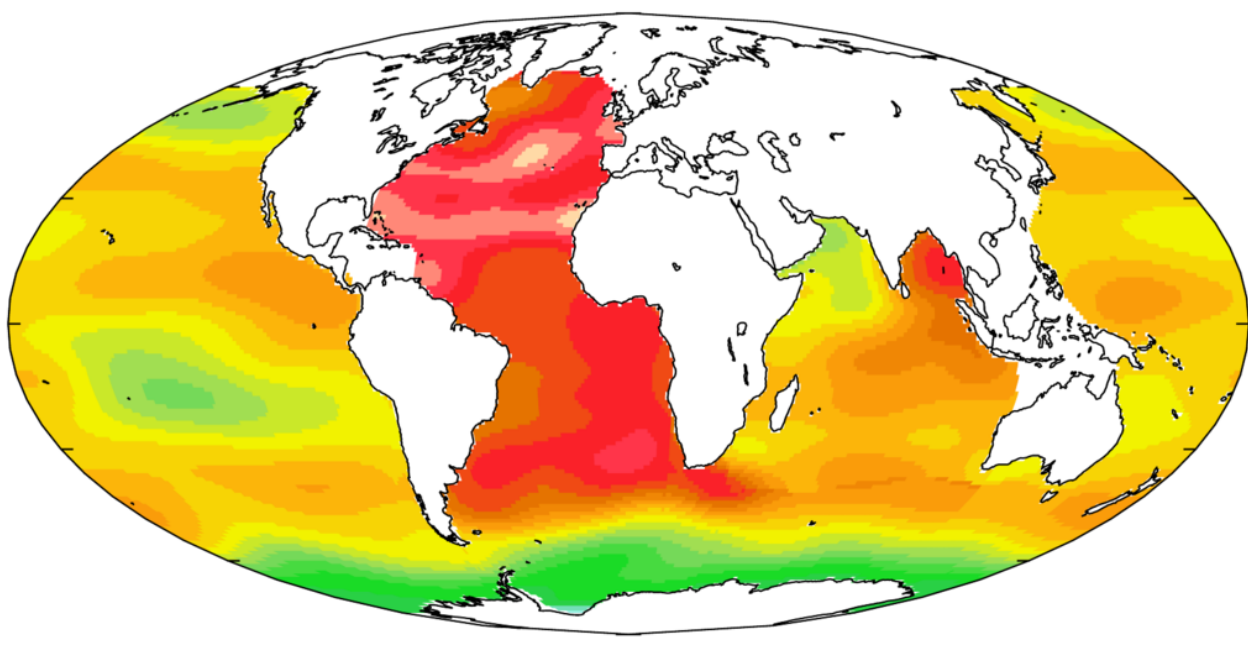

Sea-surface anthropogenic $\mathrm{CO}_{2}\left[\mathrm{~mol} \mathrm{~m}^{-3}\right]$

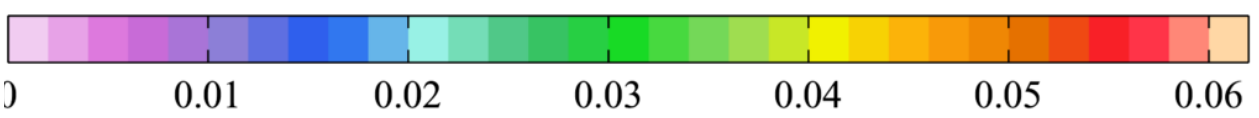

Figure 35: The distribution of $\mathrm{CO}_{2}$ in the oceans.

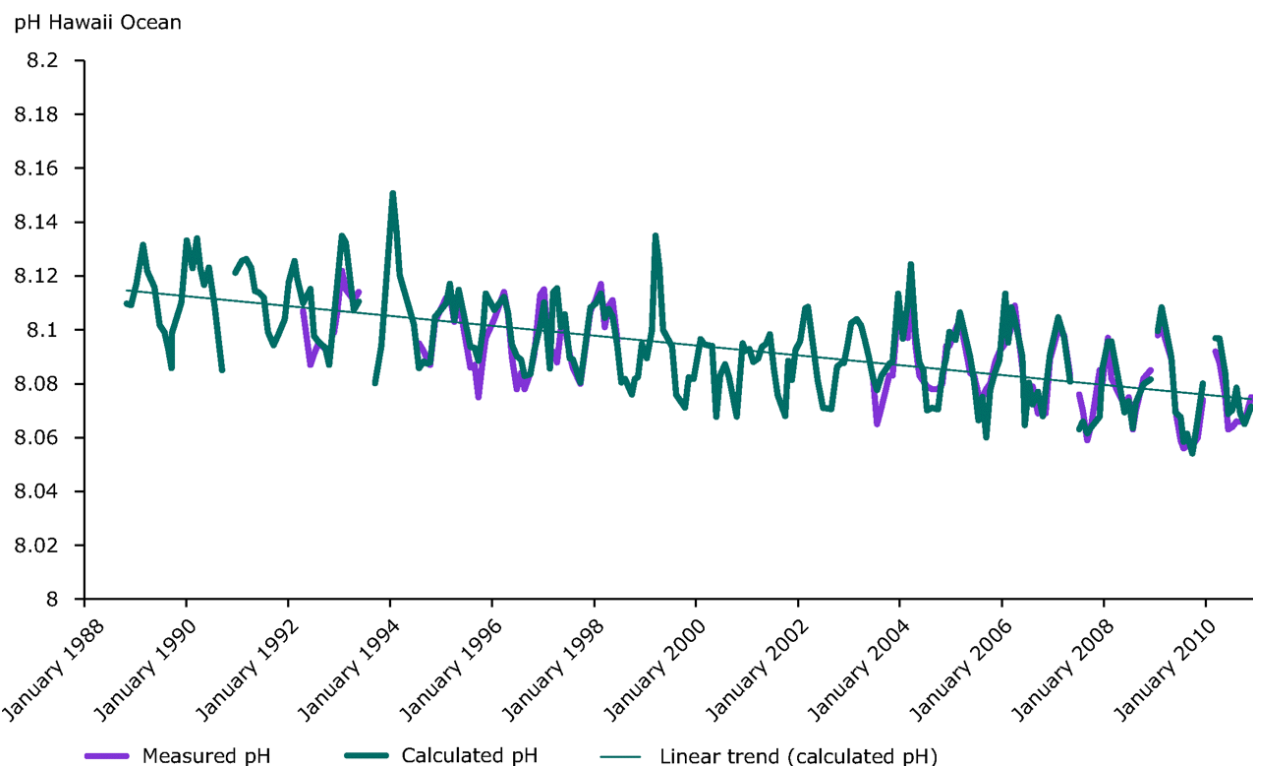

Figure 36: The seasonal changes in the sea's $\mathrm{pH}$ as measured in Hawaii. 


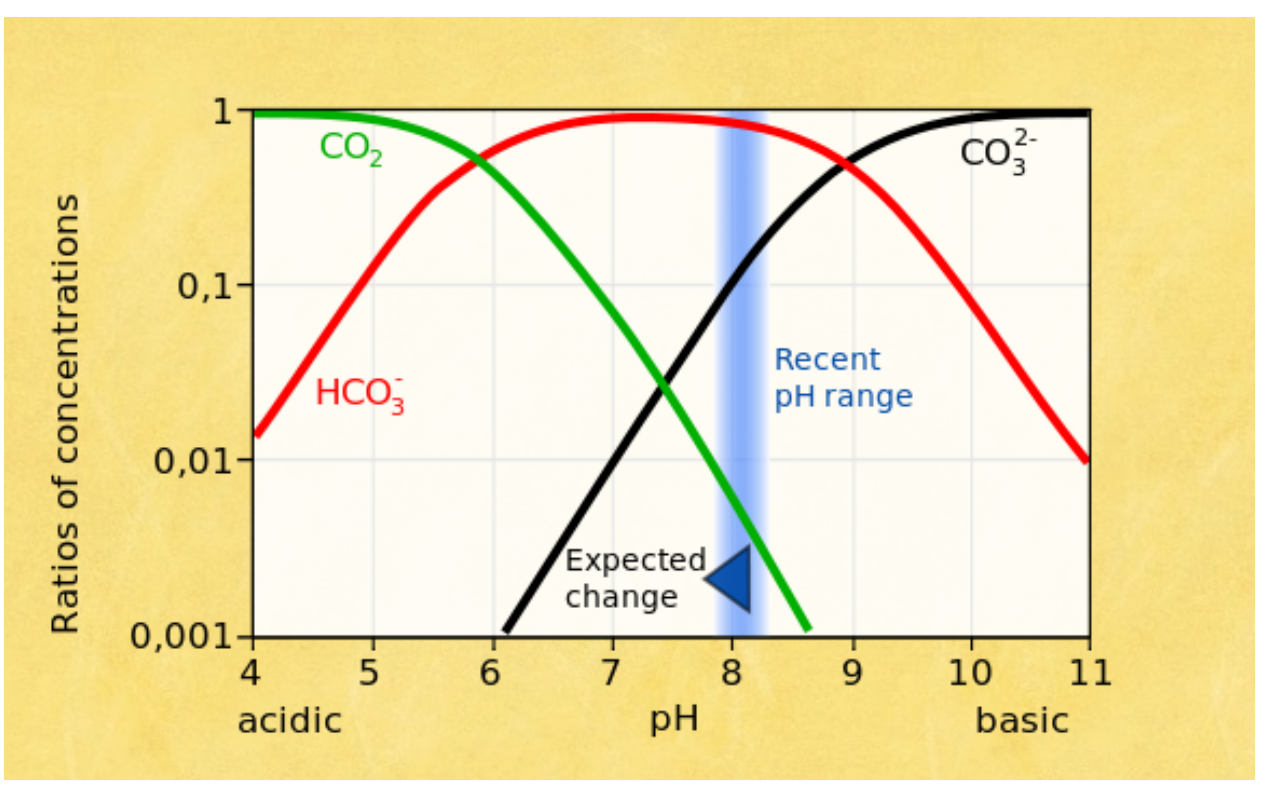

Figure 37: The phase map of $\mathrm{CO}_{2}$

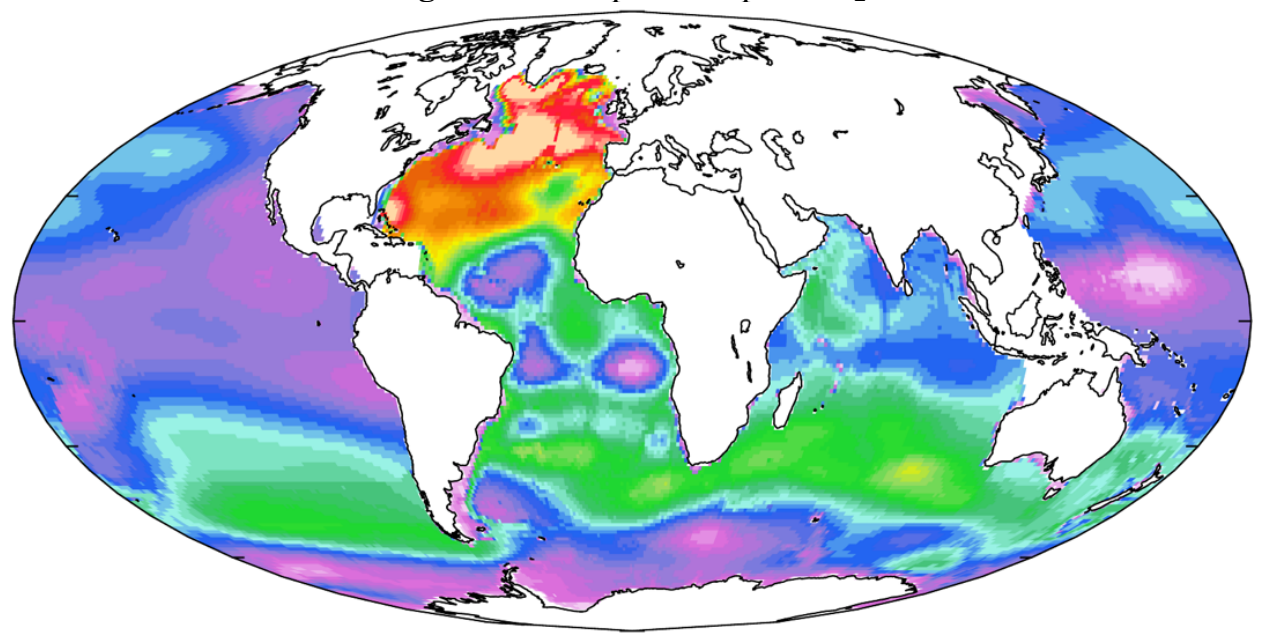

Vertical inventory of anthropogenic $\mathrm{CO}_{2}\left[\mathrm{~mol} \mathrm{~m}^{-2}\right]$

Figure 38: The column mass of $\mathrm{CO}_{2}$ in the oceans.

hence we cannot provide a confident estimate on the total contribution of $\mathrm{CO}_{2}$ and $\mathrm{CH}_{4}$ as well as other gases, to the world balance. 


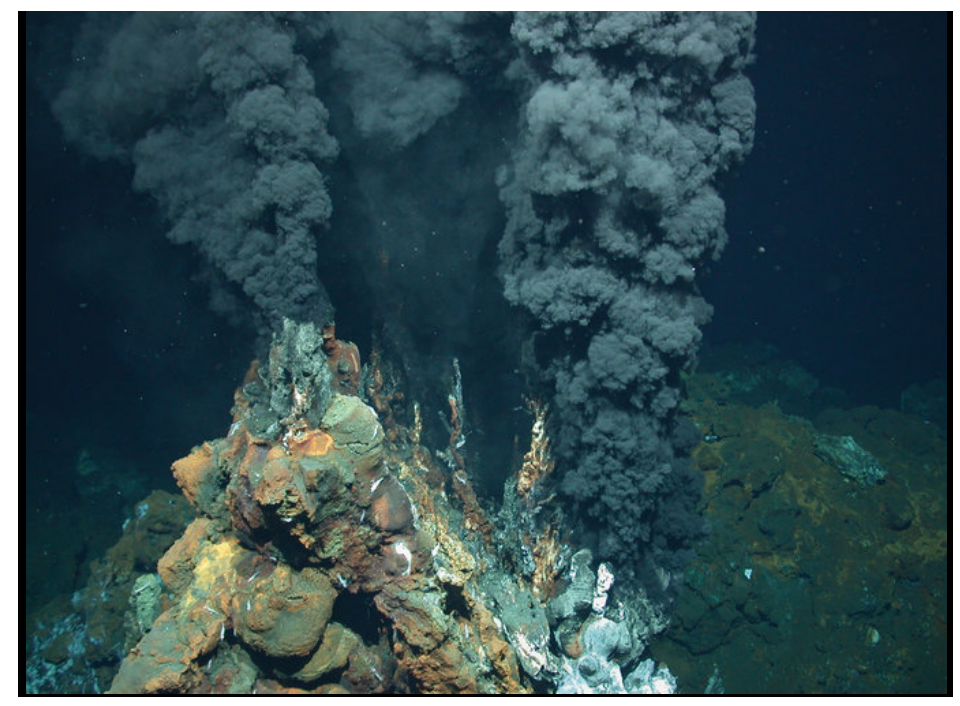

Figure 39: An underwater volcano emitting $\mathrm{CO}_{2}$.

\section{The dependence of $T(t)$ for a single place.}

So far we discussed global quantities and the difficulties in obtaining reliable averages. We now turn to the evolution of the temperature at a given location. To that goal we direct our attention to the physics of ice cores.

An ice core is a core sample that is typically removed from an ice sheet, most commonly from the polar ice caps of Antarctica, Greenland or from high mountain glaciers elsewhere. As the ice forms from the incremental buildup of annual layers of snow, lower layers are older than upper, and an ice core contains ice formed over a range of years. The ice contains inclusions of air from the time of its solidification and the air in these inclusions provide information about the properties of the atmosphere at the time of solidification. Depending on the amount of ice, the geological time can go back up to above one billion years. This enables the reconstruction of local temperature records and the history of atmospheric composition ${ }^{14}$.

Ice cores contain an abundance of information about climate at the times each inclusion was sealed by melting ice. Inclusions in the snow of each year remain in the ice, such as wind-blown dust, ash, pollen, bubbles of atmospheric gas and radioactive elements with sufficiently long lifetime. The variety of climatic proxies is significantly greater than in tree rings for example.

The length of the record depends on the depth of the ice core and varies from a few years up to 800,000 years. The time resolution depends on the amount of annual snowfall and reduces with depth as the ice compacts under the pressure of layers accumulating on top of it. The extraction of the ice core and the analysis is not simple because of motions in the ice and the fact that they are removed from the high pressure when resting deep in the ice to low pressure in open air.

An ice core from the right depth can be used to reconstruct an uninterrupted and detailed climate record extending over hundreds of thousands of years. Our discussion here relates to ice

\footnotetext{
${ }^{14}$ Bender, M.; Sowers, T.; Brook, E. (1997). "Gases in ice cores". Proceedings of the National Academy of Sciences. 94 (16): 8343Đ9.
} 
cores extracted above the Vostok lake as shown in fig. 40 and are the longest ice cores available and hence oldest.

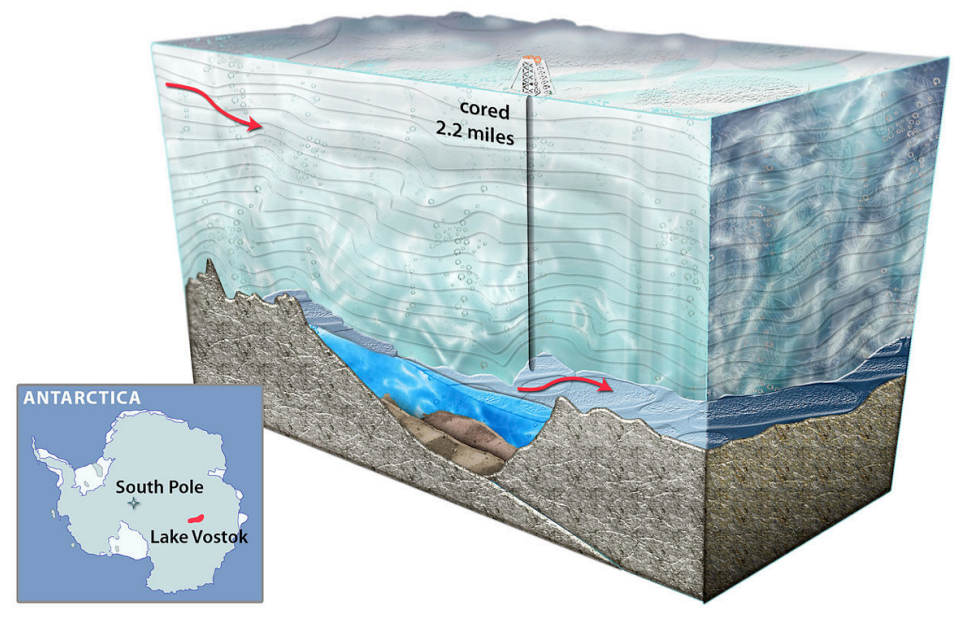

Figure 40: The location of the Vostok station in Antarctica above the lake. The total depth of ice amount to about $3.5 \mathrm{~km}$.

In fig. 41 we see the time dependence of the concentration of $\mathrm{CO}_{2}$ as a function of time for the past $800,000 y r s$. This is the longest available record. We find that the $\mathrm{CO}_{2}$ shows periodic behavior with a periodicity of about 143 million years. The minima reach $170 \mathrm{ppm}$ while the peak reach about 300ppm, more than the value at the beginning of the industrial era. The temperature variation in the past 800,000 years are shown in fig. 43. We find that (a) the past temperature shows that same oscillations as the $\mathrm{CO}_{2}$ as well as the other gases. Also, the minima in the temperature were significantly lower (about $8^{\circ} \mathrm{K}$ ) than present day temperature and the peaks higher (by about $3^{\circ} \mathrm{K}$. Fig. 42 shows the temperatures in recent history. The Greek period in history coincides with high (global) temperatures. The climate about 20,000 years ago was quite cold.

Fig. 43 is one of the most crucial ones. We see how $\mathrm{CO}_{2}$ (in blue), the temperature (in red), $\mathrm{CH}_{4}$ (in green) and ${ }^{18} \mathrm{O}$ change simultaneously with time. The critical question is what is the driver of the changes. This question splits into two questions: What caused the first agent to change and who is the first agent, does a change in $\mathrm{CO}_{2}$ drive the variations in $\mathrm{CH}_{4}$, the temperature and ${ }^{18} \mathrm{O}^{15}$ for example? It is inconceivable that a change in $\mathrm{CO}_{2}$ caused a change in $\mathrm{CH}_{4}$. It is physically and chemically simpler to assume that a change in the temperature drove the change in the concentration of all gases. Note that the time difference between the peaks is very short and hardly noticeable. We know from daily physics that solubility decreases with temperature and hence as the temperature rises all gases leave the solution (the sea).

A check on fig. 44 shows again that we are today in a warm period and just 20,000 ago the Earth experienced a cold period. The entire amplitude in the temperature oscillations is less than $10^{\circ} \mathrm{C}$.

\footnotetext{
${ }^{15}$ The diffusion of this isotope indicates water diffusion
} 


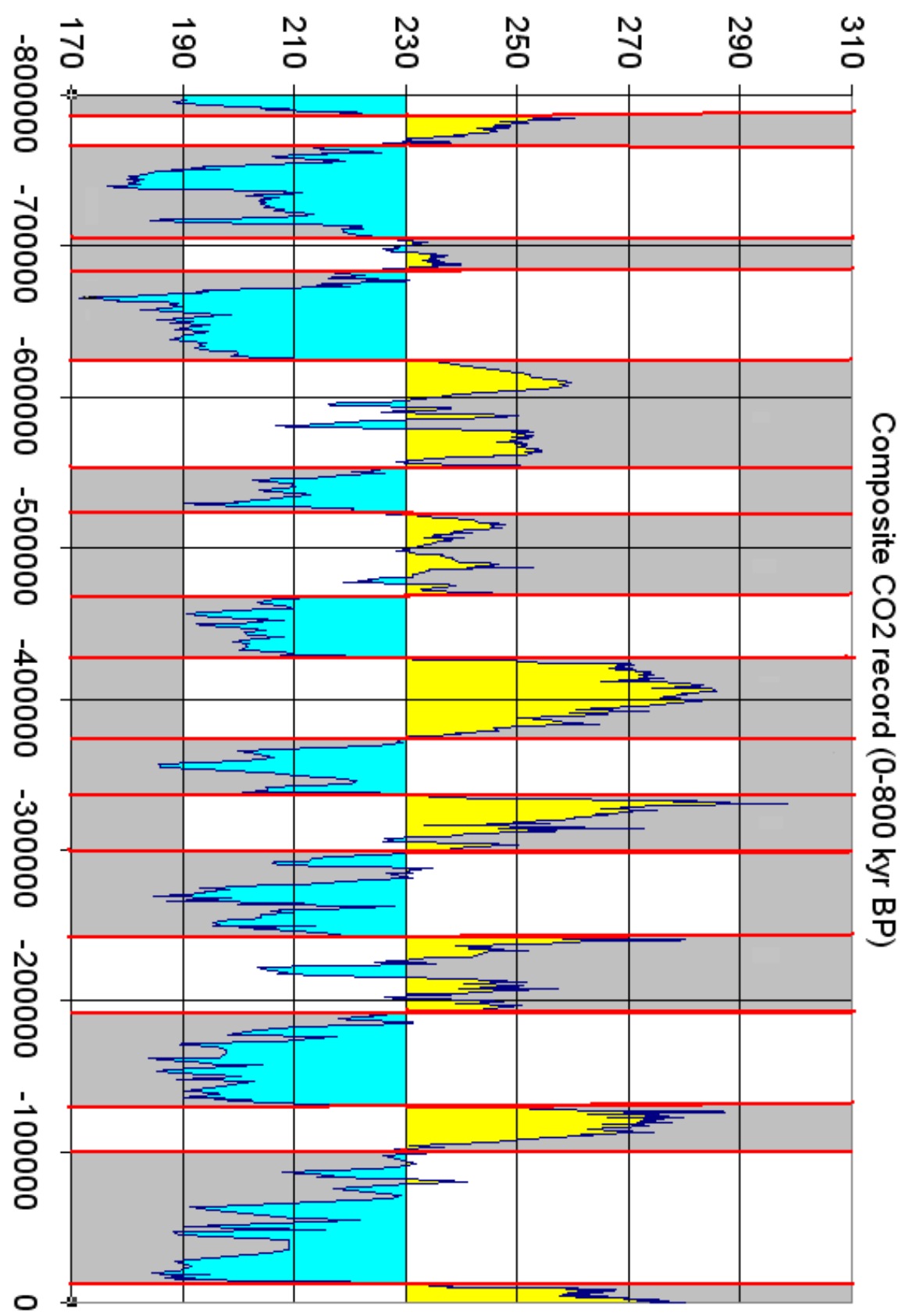

Figure 41: The time variation of the $\mathrm{CO}_{2}$ concentration for the past 800,000yrs. Please note that the time axis goes backwards. 


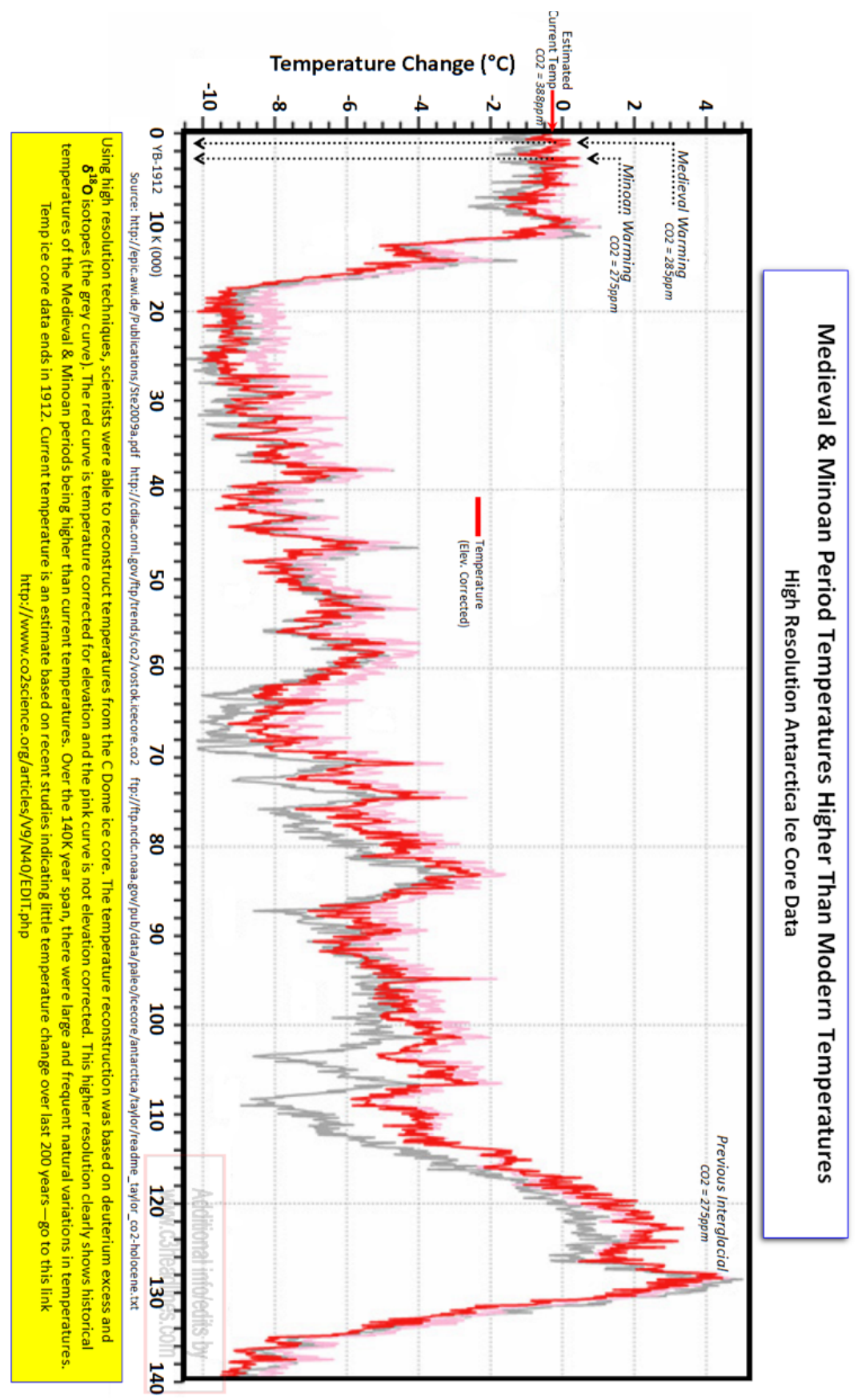

Figure 42: The time section which corresponds to the Minoan and Medieval eras. Note the the horizontal time axis run right to left. We see cold and warm periods in recent history. 


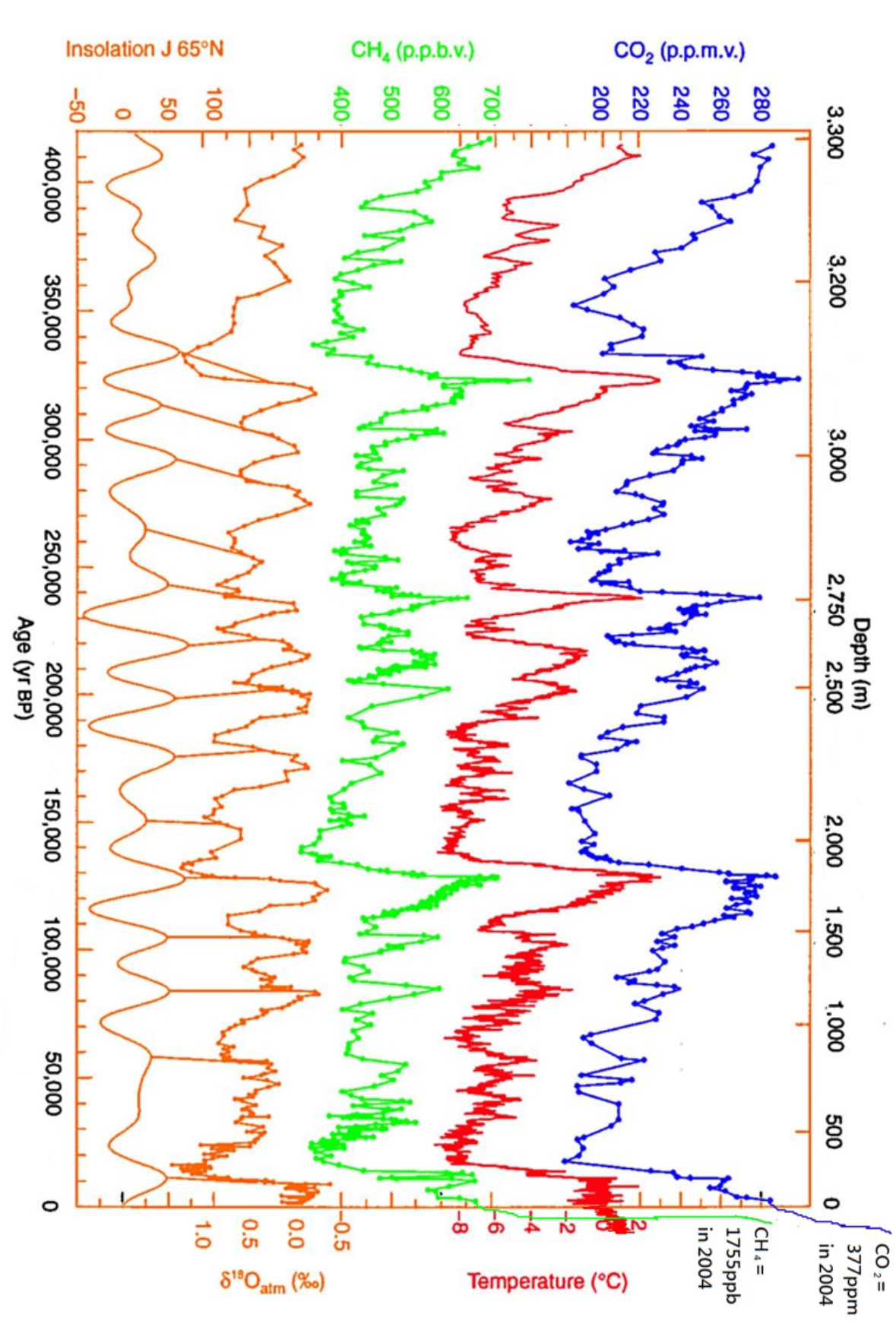

Figure 43: The variation of the concentration of $\mathrm{CO}_{2}$ (in blue), the temperature (in red), $\mathrm{CH}_{4}$ (in green) and ${ }^{18} \mathrm{O}$ with time. 


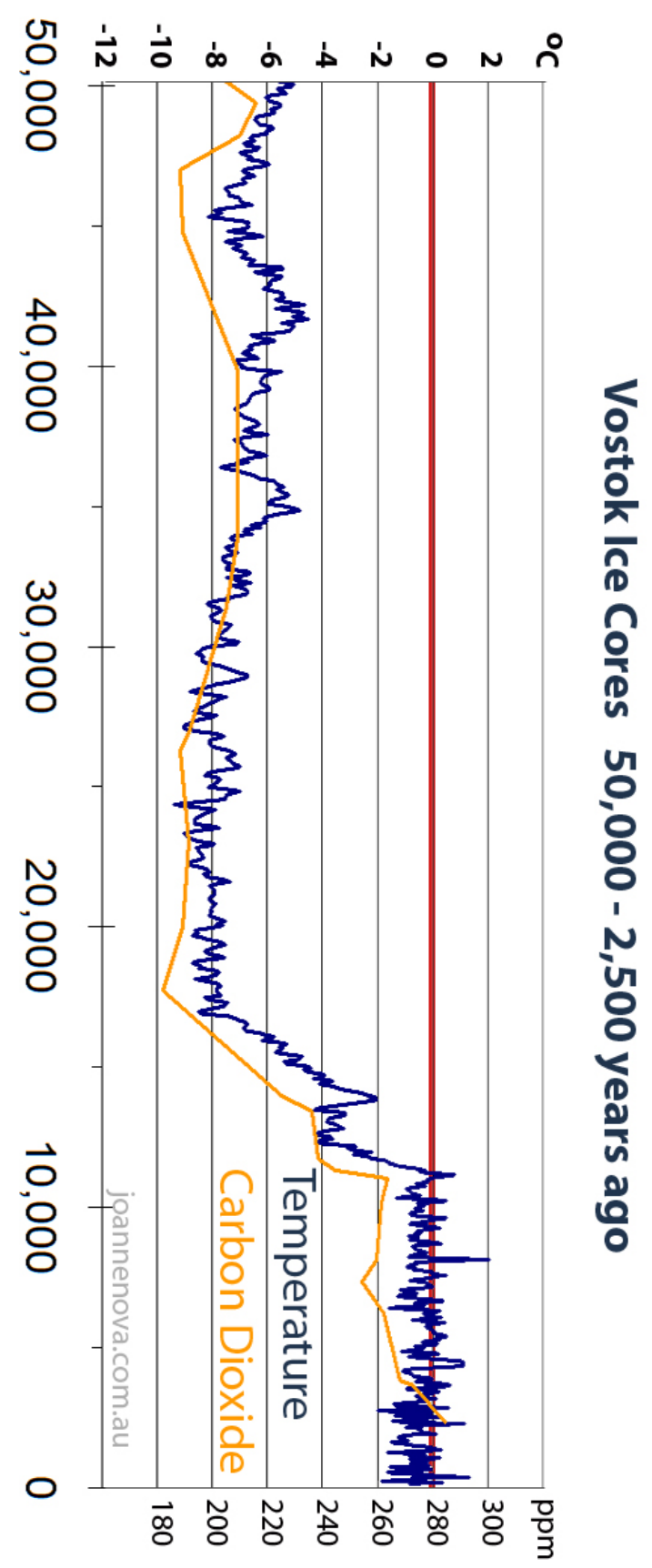

Figure 44: Recent record of the temperature (in Vostok) showing the cold past period and the recent heating. We are today in a warm period. About 20,000 years ago there was on Earth a long period of lower than today temperatures. 


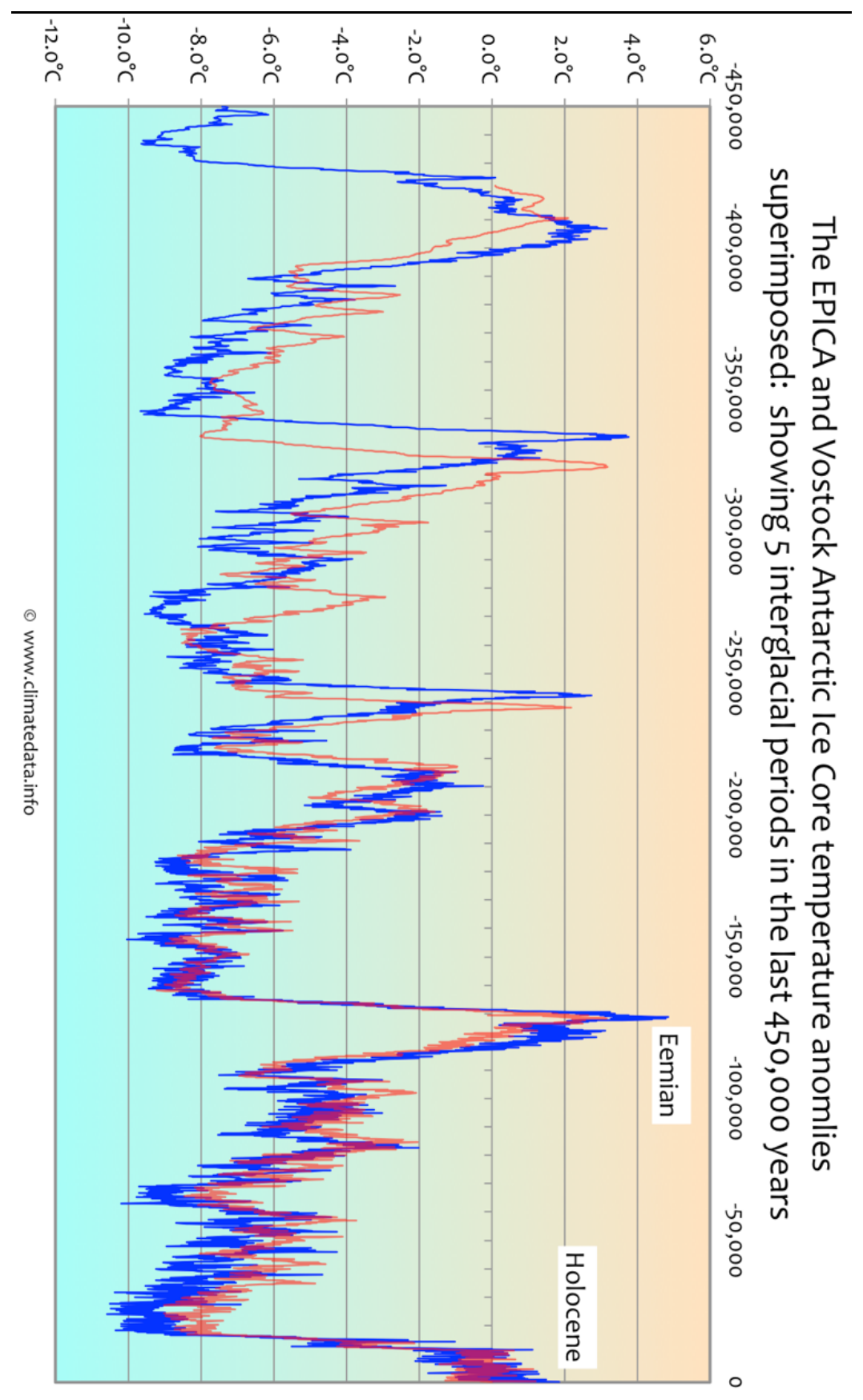

Figure 45: The comparison between the variations in two sites, the EPICA (The European Project for Ice Coring in Antarctica) and Vostok. 
The comparison between two independent stations for ice cores is shown in fig. 45. EPICA is an acronym for a multinational European Project for Deep Ice Core drilling in Antarctica, the European Project for Ice Coring in Antarctica and they drill the ice at Dome Concordia and Kohnen Station. We see a perfect agreement between the two independent results along with a time difference.

Fig. 46 demonstrate the existing correlation between the $\mathrm{CO}_{2}$ concentration and the temperature. The temperature scale (in Fahrenheit) shows maximum temperature of about $\approx 2.8^{\circ} \mathrm{C}$ above present day temperature. On the other hand, present day $\mathrm{CO}_{2}$ increases to about $370 \mathrm{ppm}$. This trend of rising $\mathrm{CO}_{2}$ and stagnant temperature is seen to continue past the last year shown in the graph. In other words a fast rise in $\mathrm{CO}_{2}$ concentration does not show any signs in the temperature.

An attempt to correlate the temperature changes with the Milankovich cycles is shown in fig. 47. We see that the temperature performs oscillations. The oscillations have several periodicities implying several drivers. One of the medium range drivers is the Milankovich oscillations, namely the few percent periodic changes in the orbit of the Earth. The figure shows a correlation between a combination of Milankovich cycles. We see some agreement between the theory and temperature variation, but not a perfect one.

Fig. 48 shows the correlation between the changes in temperature and $\mathrm{CO}_{2}$. The time difference is small, about $10^{3}$ years (not always observable in the very old ice cores) and the $\mathrm{CO}_{2}$ lags behind the temperature. In other words, it is the rise in temperature which causes the rise in $\mathrm{CO}_{2}$ and not vice versa. This is a simple physical explanation, namely a heated solution loses dissolved gases to the atmosphere.

We learn also that the climate is stable, there is no $\mathrm{CO}_{2}$ runaway. If the basic assumption is that a rise in the concentration of $\mathrm{CO}_{2}$ heats the atmosphere, then there must be an agent which neutralizes most of the effect of $\mathrm{CO}_{2}$ and give rise to the stability.

Fig. 49 summarizes the different variations/oscillations of the temperature and consequently the different cycles experienced by the atmosphere. It is conceivable that what we observe presently in our atmosphere is nothing but one of the cycles the Earth atmosphere experiences. In fig. 50 we see the 143 million cycle of glaciation. The ice expanded on Earth from the poles to about latitude 35. Finally we show in fig. 51 the various cyclical patterns of the Earth's temperature.

\section{Theoretical Predictions of the Global Temperature}

There are many numerical Global Circulation models used to predict Earth's climate. As the problem is very complicated giant codes were developed to predict the Global Temperature and these models apply different approximations and hypothesis. We present a typical comparison between several models. As a matter of fact any such comparison is equally good to demonstrate the basic problem: The failure of the theory even to predict a decade forward. We show in Fig. 52 the prediction of different model listed in table $1^{16}$. The comparison between the model predictions of the vertical structure and the measurements are shown in fig. 53 and we see a clear discrepancy. The reality falls below all the predictions. It should be noted that the lion share of the difference between the theory and observation is due to the 'pause' in the temperature rise (see

\footnotetext{
${ }^{16}$ Climate Change 2001: Working Group I: The Scientific Basis
} 


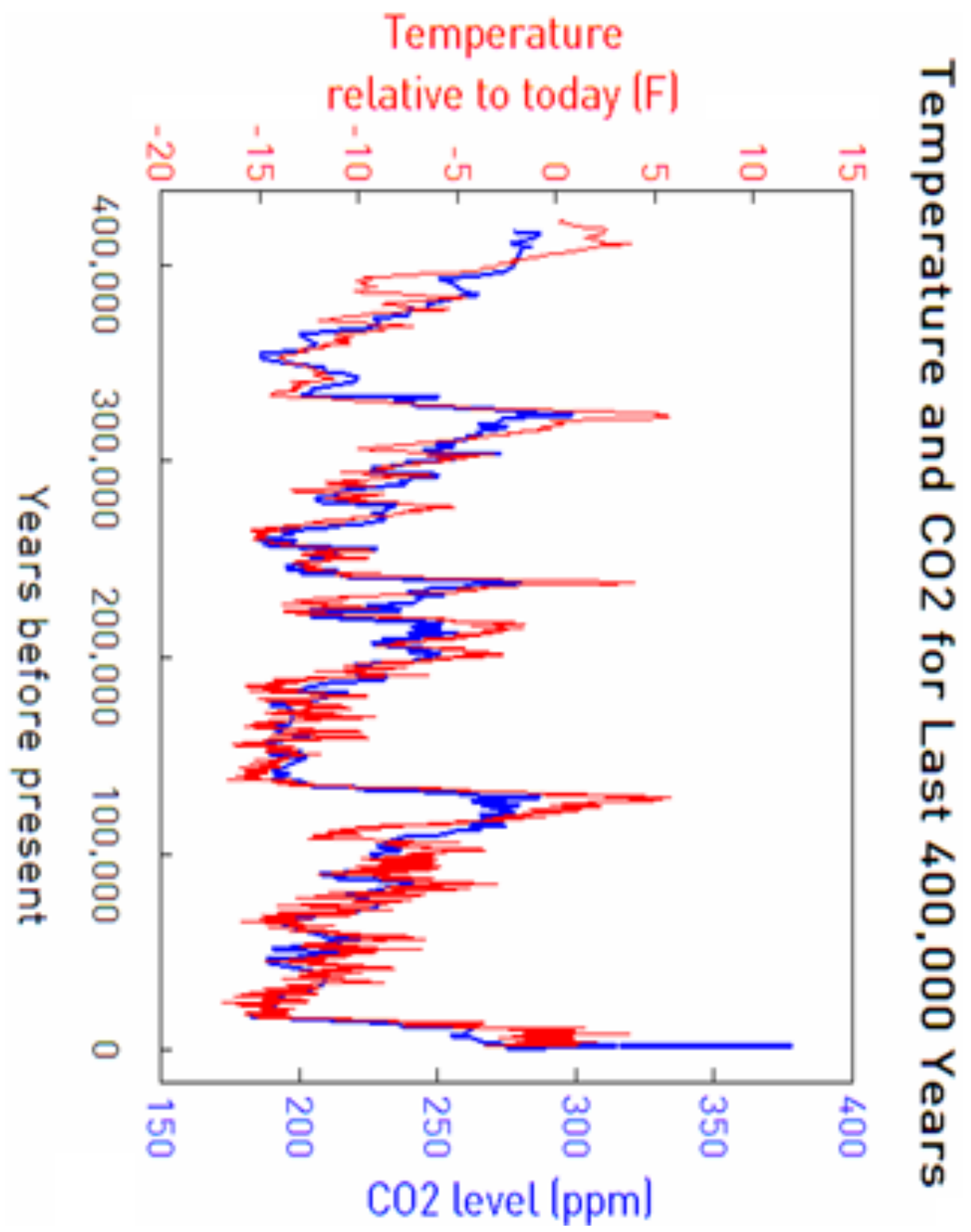

Figure 46: The correlation between the concentration of $\mathrm{CO}_{2}$ and the temperature. 


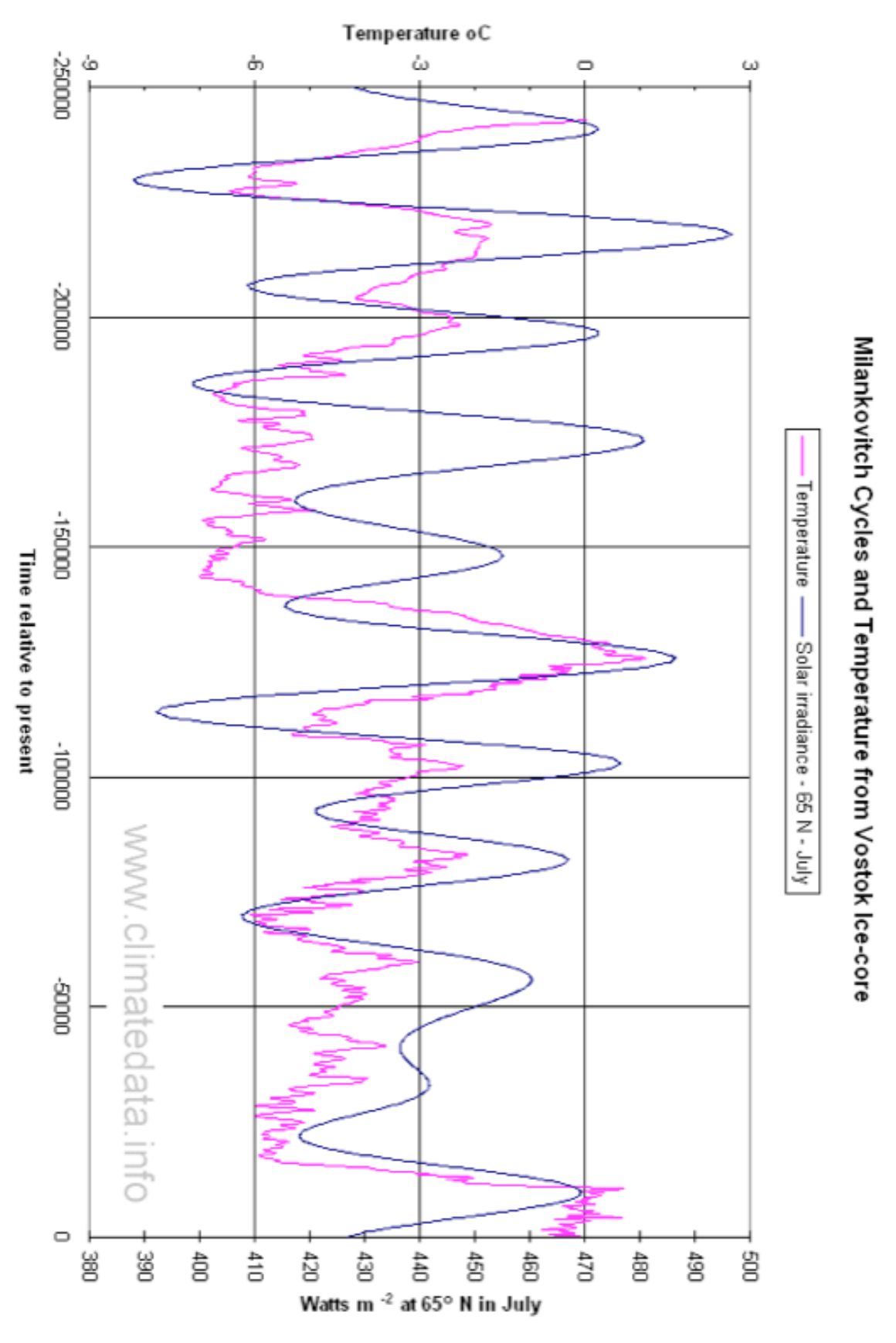

Figure 47: The correlation between the $T(t)$ and the Milankovich cycles. 

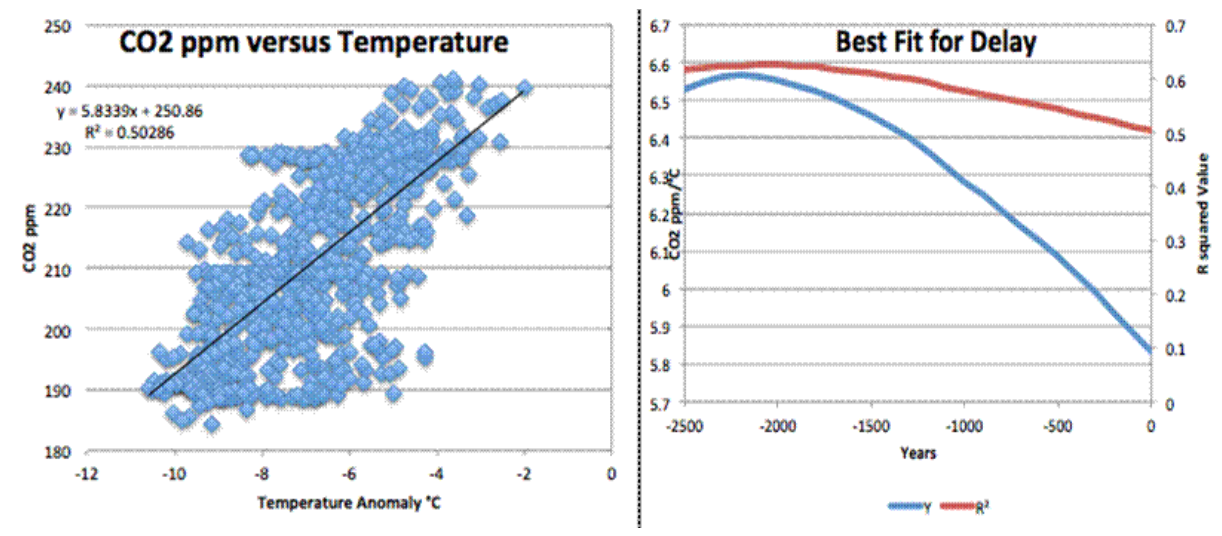

Figure 48: The correlation between the change in temperature and $\mathrm{CO}_{2}$.

later). The agreement between the theory and observation improves but the disagreement is not fully eliminated when comparison is made after neutralizing the effect of the hiatus.

Table 1: Comparison of Global Circulation Model predictions (taken from Temperature Projections IPCC/Hansen n Non- $\mathrm{CO}_{2}$

\begin{tabular}{|l|l|}
\hline Author & Method \\
\hline IPCC & $\mathrm{CO}_{2}$ based model (2007) \\
James Hansen & $\mathrm{CO}_{2}$ based model (1988) \\
Nicola Scafetta & based on astronomical harmonic model (2011) \\
Don Eastbrook & based on multi-decadal oscillations (2001) \\
Syun-ichi Akasofu & based on multi-decadal oscillations (2008) \\
Patrick Michaels & straight line regression on the last 30 years (2008) \\
Joe the Actuary & sine wave regression on HadCRUT data (2009) \\
Alan Cheetham & based on visual recurrent cycles from HadCRUT data (2009) \\
Roger the Tallbloke & based on regression on AMO,SOI,CO2,SSN (2012) \\
\hline
\end{tabular}

We should note at this point, that the IPCC has almost routinely cut its prediction if the effect of global-warming, implicitly rejecting the models on which it once so heavily relied on in warning mankind. In the second draft of the Fifth Assessment Report it had broadly agreed with the models that the world will warm by $0.4-1.0 \mathrm{C}^{\circ}$ from 2016-2035 against 1986-2005. But in the final draft it quietly cut the 30 -year projection to $0.3-0.7 \mathrm{C}^{\circ}$. Should we be worried by the fact that different theoretical model differ so much? Where is the error? 


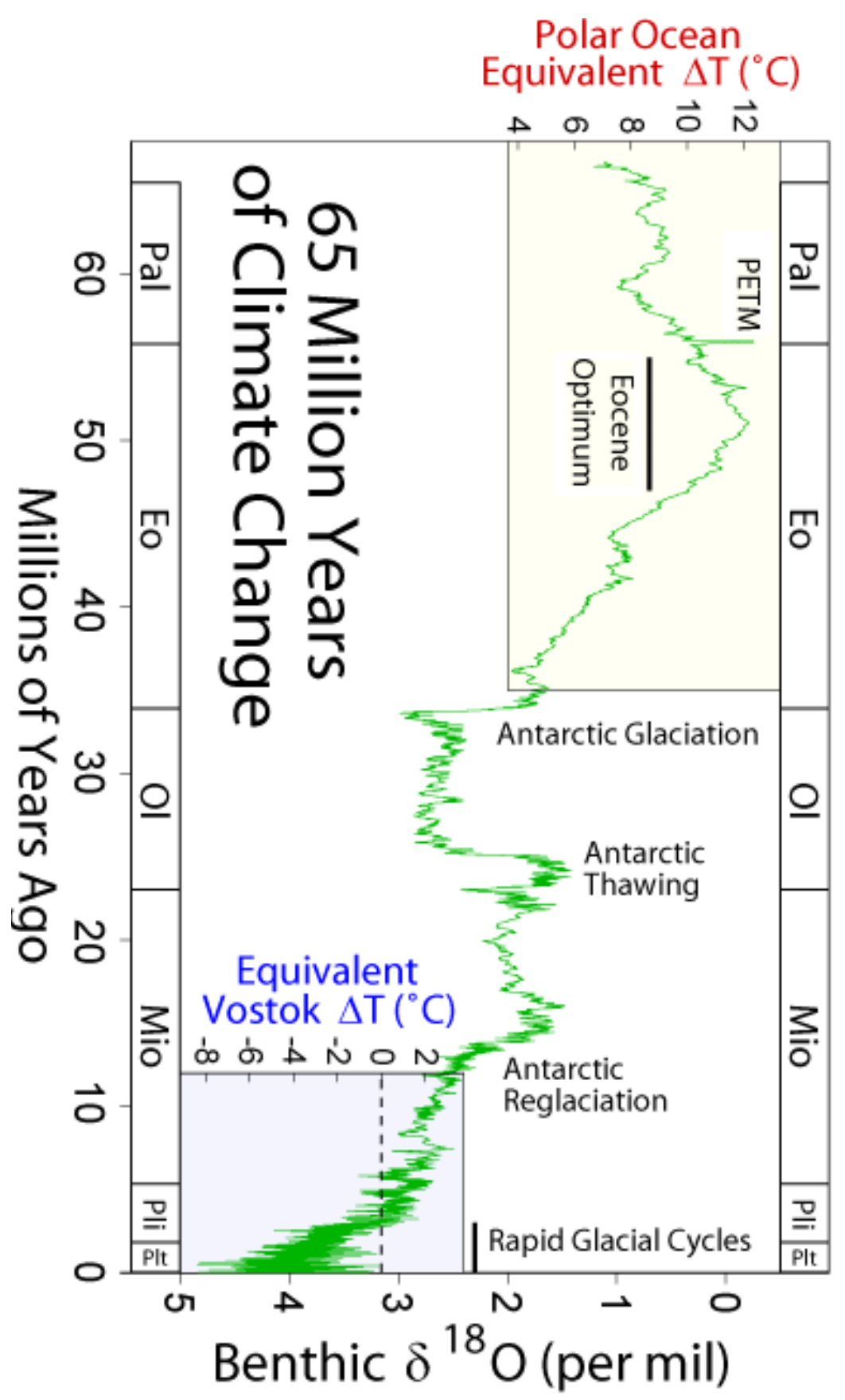

Figure 49: A summary of the multitude of temperature cycles experienced by the atmosphere. 


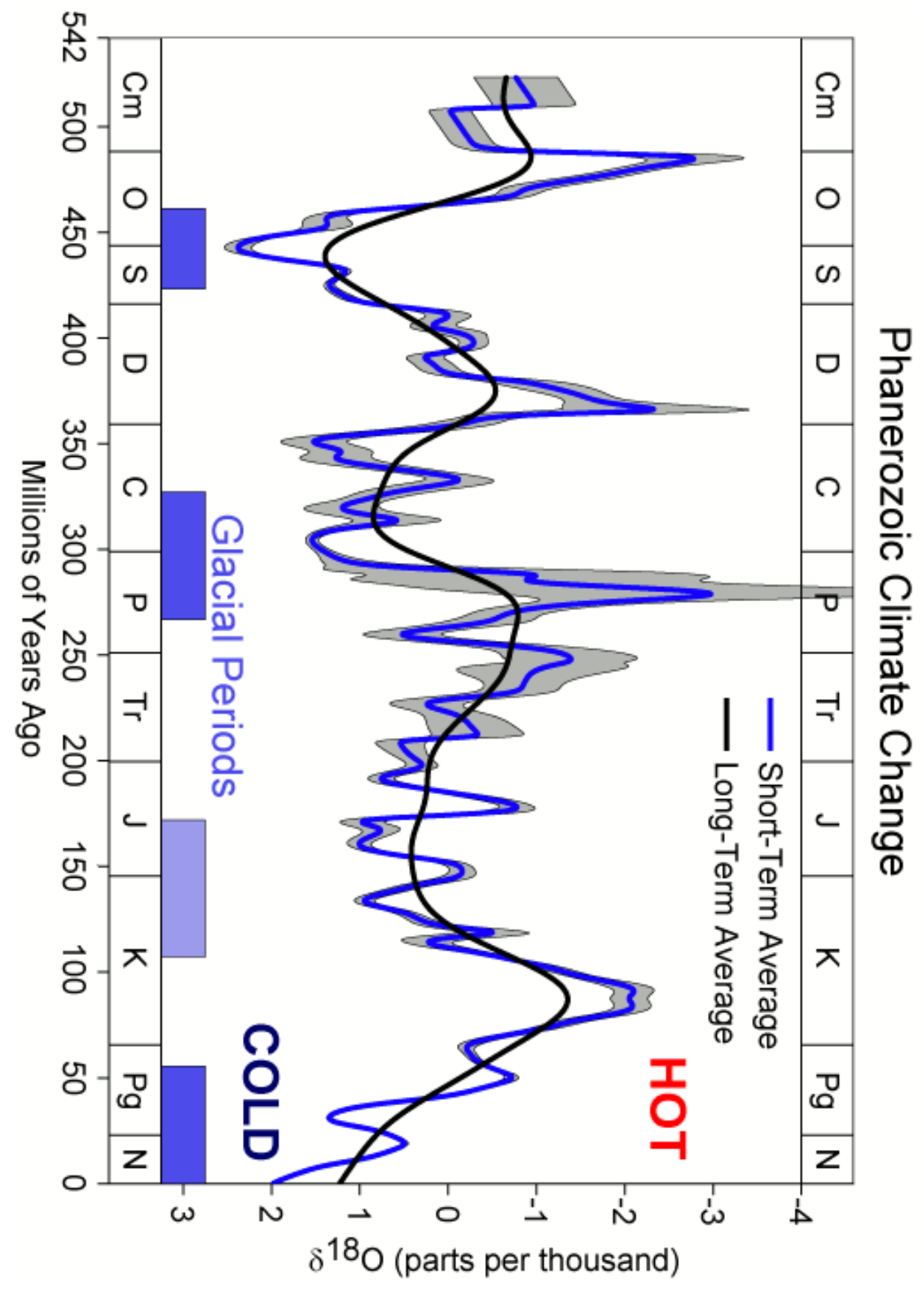

Figure 50: The change of the temperature over 143 million years and glaciations. 


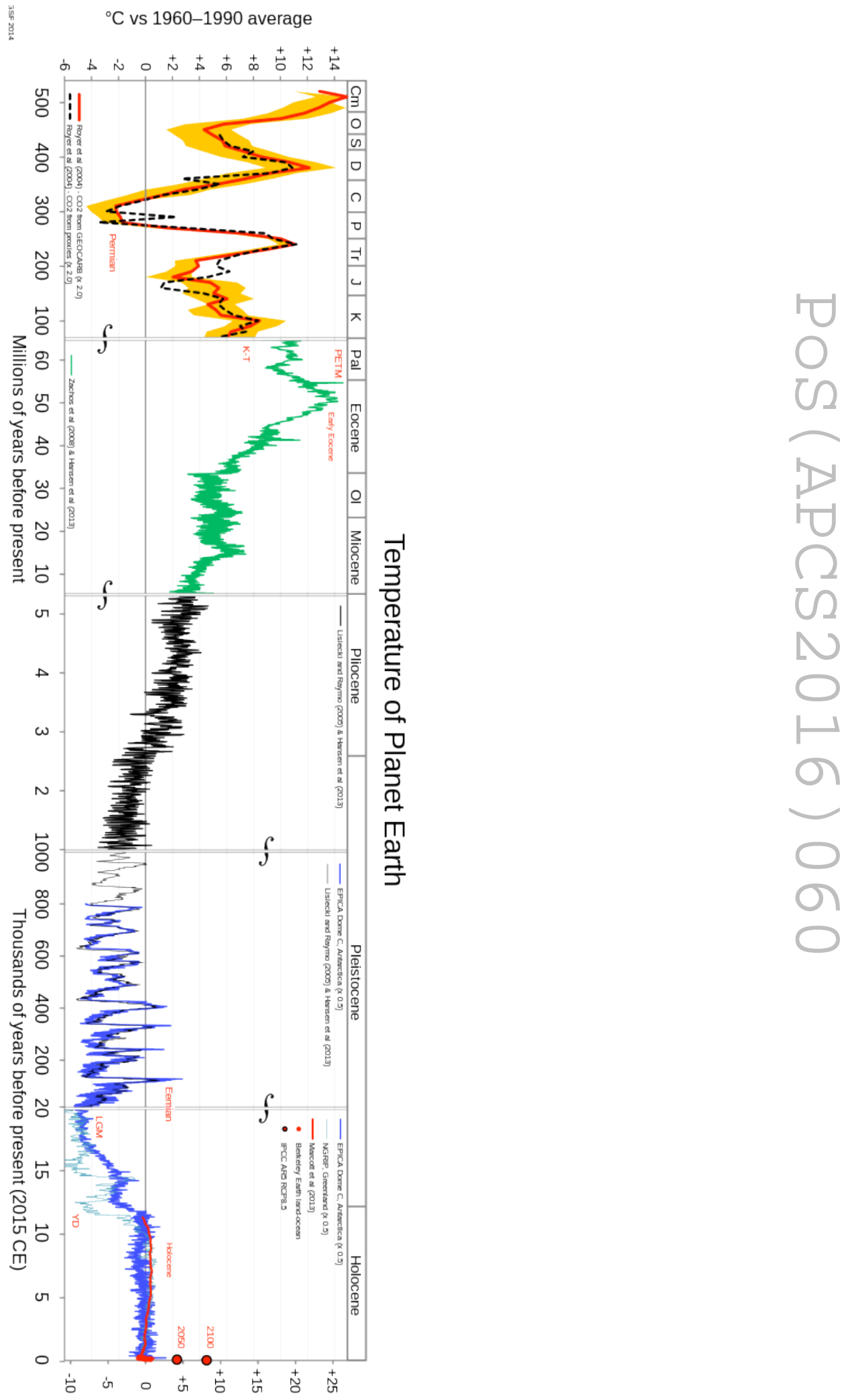

Figure 51: The various cyclical patterns of the Earth's temperature. 


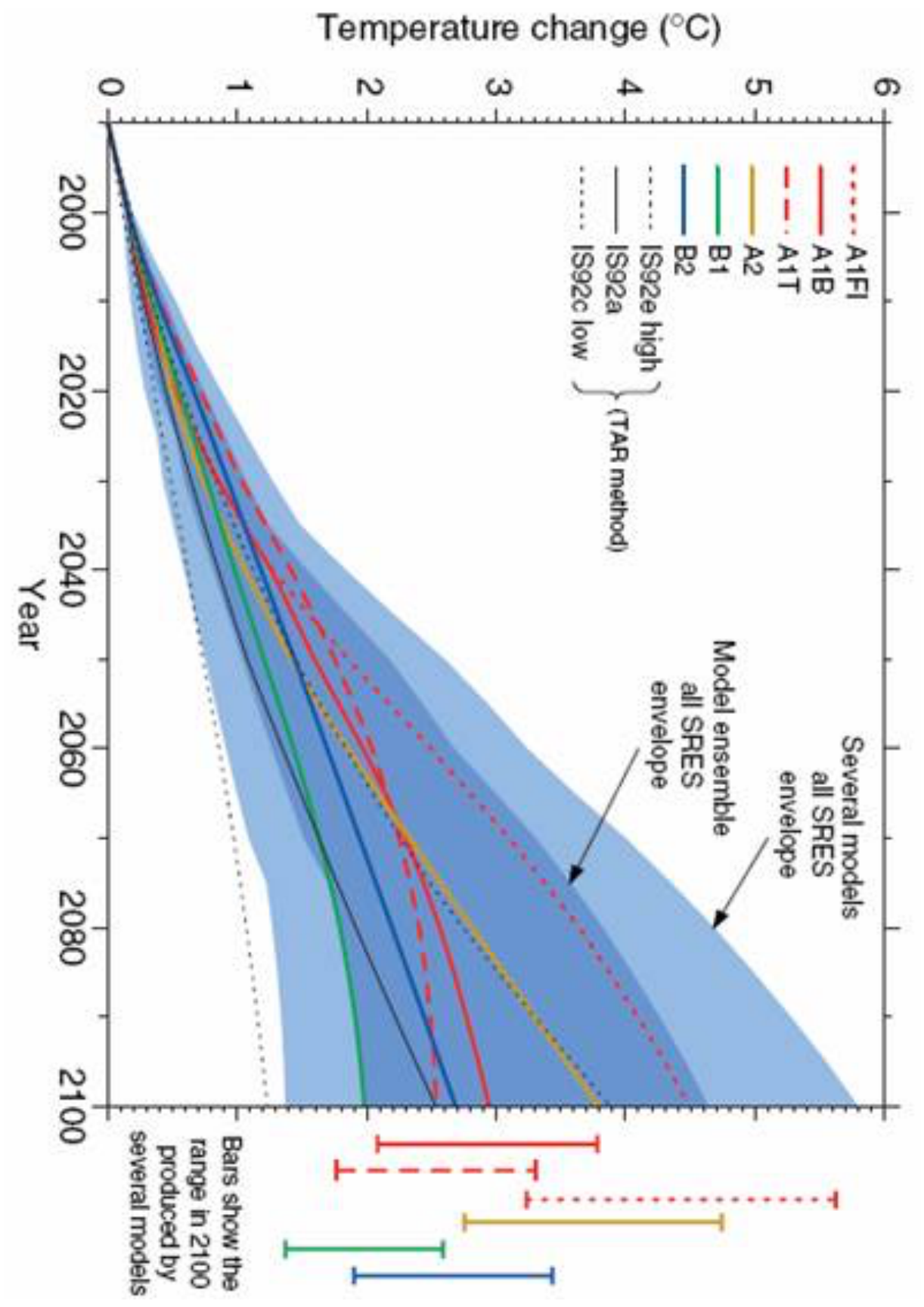

Figure 52: A comparison between the temperature of the troposphere and the stratosphere. 


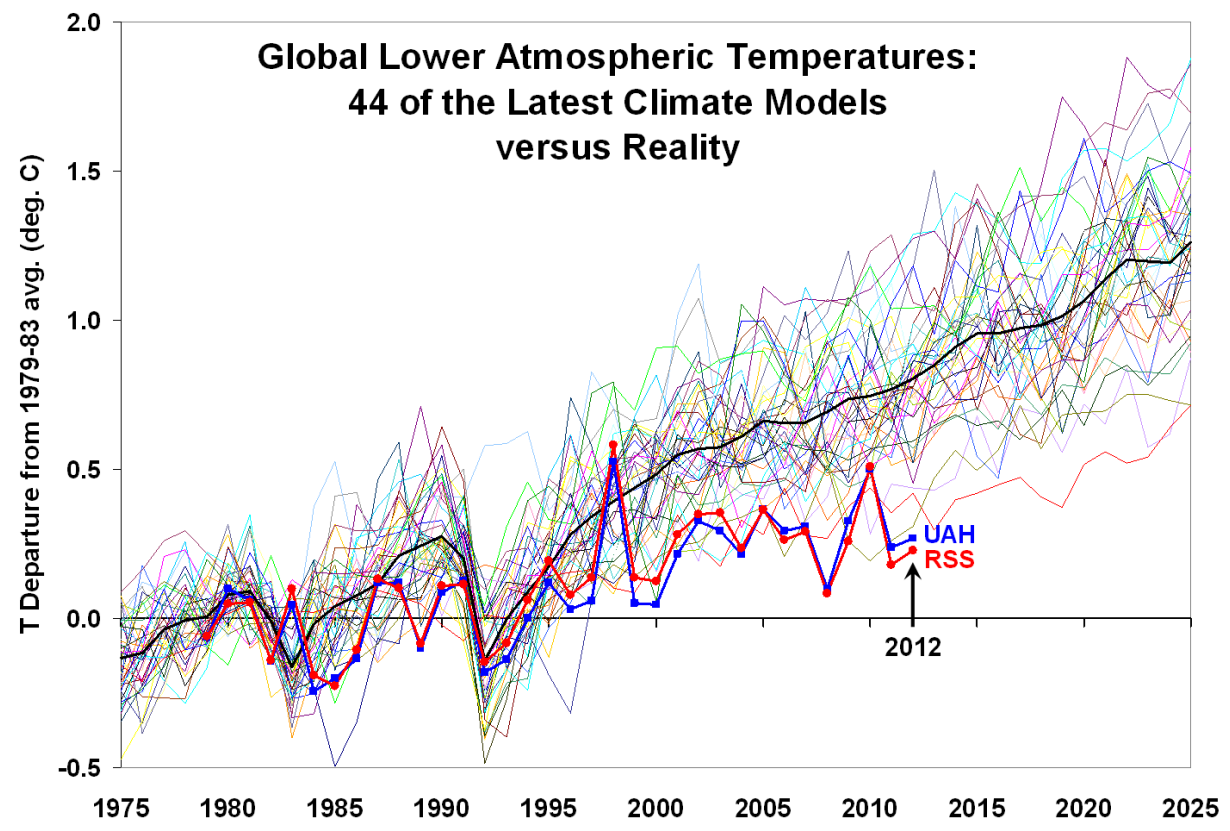

Figure 53: A comparison between the predicted global temperature change and the observed value. The curves marked UAH and RSS are the observations.

\section{The Interaction of the Sun with the climate on Earth}

We show in fig. 55 the number of sun spots in the various cycles since the discovery of the sunspots by Galileo. Also plotted is the mean Earth temperature. The Maunder minimum is easily spotted at the beginning of the observation period as a period with almost no sunspots and low temperatures. The Maunder minimum was discovered by Eddy in 1976 and was named after the discoverers Maunders (a husband and wife) at the end of the 19th century. During the Maunder minimum Europe experienced a very cold period. During this period the London Thames froze in winter, a unique event which is seen in the drawing shown in figure 56. Similar cold periods occurred also during the Dalton minimum. There were no similar cold periods in Europe and there were no other minima in sunspots. We have no information of what happened in other regions of the Earth.

It should be stressed that we are presently unable to predict the coming of a minimum in sunspots let alone its duration.

The 22nd, 23rd and the beginning of the 24th solar cycles, which the present one, are shown in fig. The implications for the coming temperature behavior seems obvious. Another support for the idea that the Sun affects the mean Earth temperature is seen in fig. 59 where the Sunspot number and the Global mean Sea Surface Temperature are shown.

Nir Shaviv discovered a strong correlation between the the times the Earth crossed the spiral arms of our galaxy and glaciation. The explanation is in short, that cosmic rays are created mostly in the spiral arms of the galaxy and then diffuse in the galaxy. Hence, the spirals have a high density of cosmic rays. This rays propagate in the galaxy and penetrate the Earth atmosphere, ionize it, 


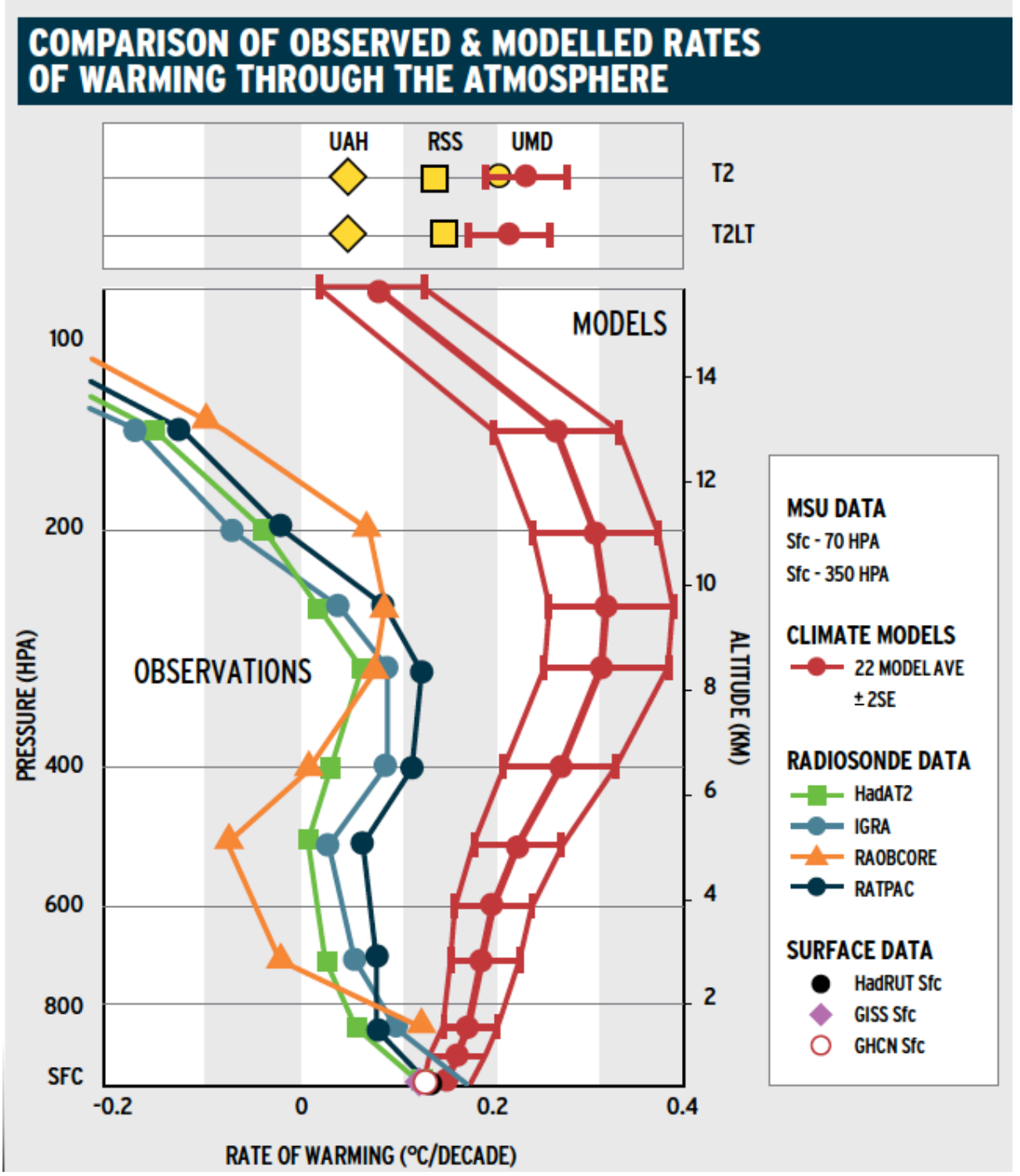

Figure 54: A comparison between the predicted and observed vertical temperature distributions. 


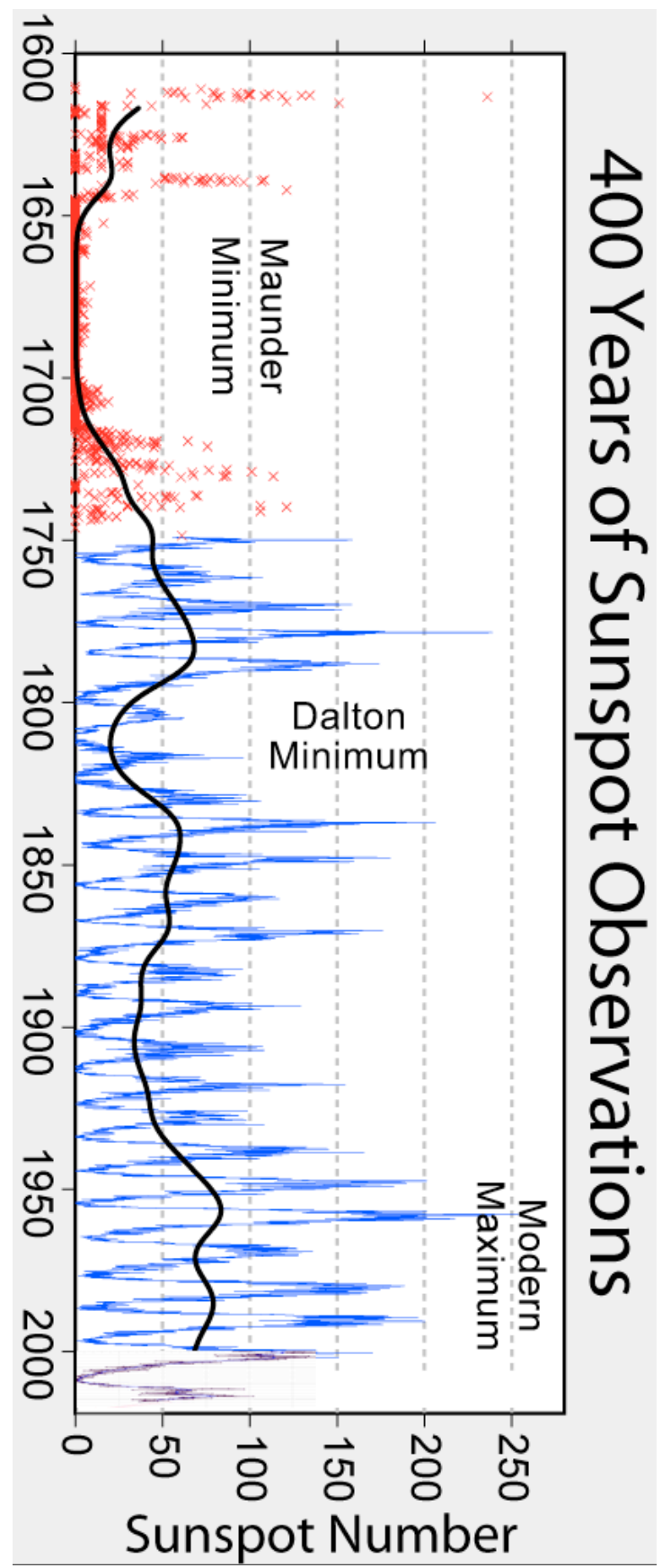

Figure 55: The sunspots cycles as known starting from the discovery of sun spots by Galileo. 


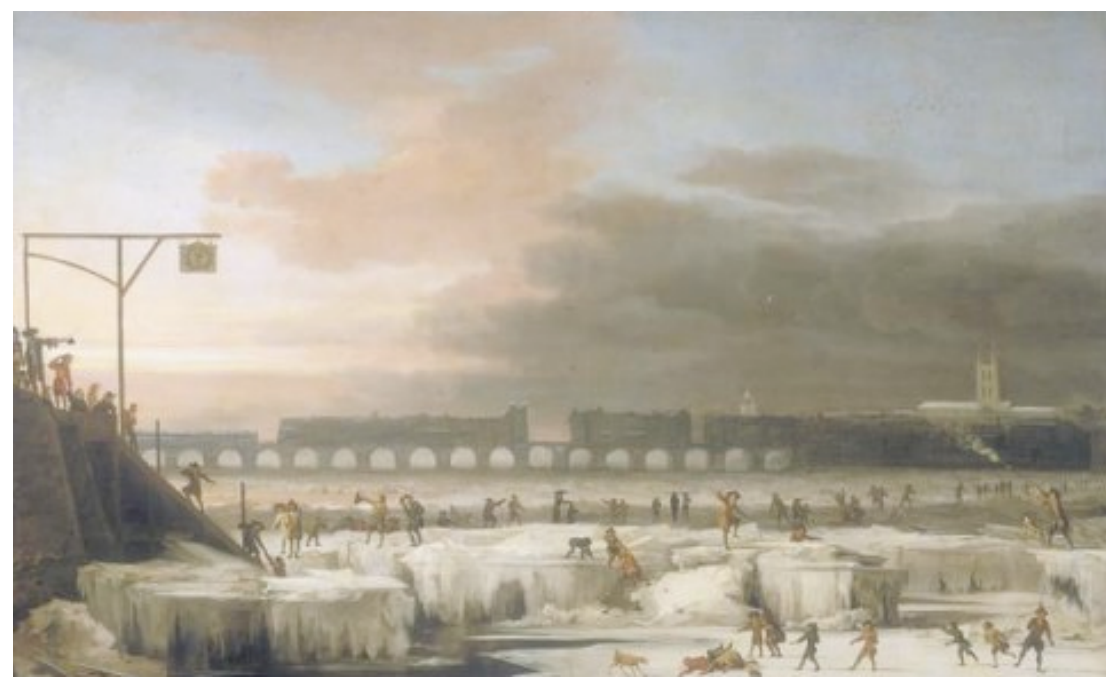

Figure 56: Drawings of the frozen Thames made during the Maunder minimum.

and increase the rate of cloud formation. The cloud cover changes the albedo of the Earth and consequently cool it.

The solar system has two basic motions in the galaxy, in the plane of the galaxy and perpendicular to it. Since the rotation of the galaxy is differential, the solar system crosses the spiral arms every about 143 million years. The rise in the cosmic ray bombardment during the passage through the arm causes ionization and nucleation in the atmosphere and increases cloud cover. As a consequence, the Earth albedo changes and more solar radiation is reflected. This decrease in solar radiation cause a spread glaciation and cold period. As can be seen from the figure, the correlation between glaciation and crossing a spiral arm is remarkable.

A similar effect is seen with the motion of the solar system in the vertical to plane of the galaxy direction as shown in fig. 60. Strong evidence for the existence of this periodicity in the data was found. In general all phenomena that change the state of ionization in the Earth atmosphere have the same effect, namely change of the albedo which in turn affect the temperature on the Earth.

\section{The temperature plateau or the 'hiatus in Global Warming'}

The observation of the Global Temperature appear to show a plateau in the temperature. The plateau is frequently called a 'pause' (as if the Global Warming takes a pause or hiatus). This can be seen from the many results given before when a long time sequence is shown or from fig. 61 when just the last 19 years are shown plus few years before the 'pause' started. The plateau extends already for 19 years. Clearly, none of the theoretical models predicts such a plateau. Moreover, the fast rise in $\mathrm{CO}_{2}$ should have prevented this plateau. Where is the extra energy absorbed by the rising $\mathrm{CO}_{2}$ stored?

If the plateau is just a fluctuation in the data, then clearly the GCM, which attempt to predict the mean global temperature, cannot predict it. 


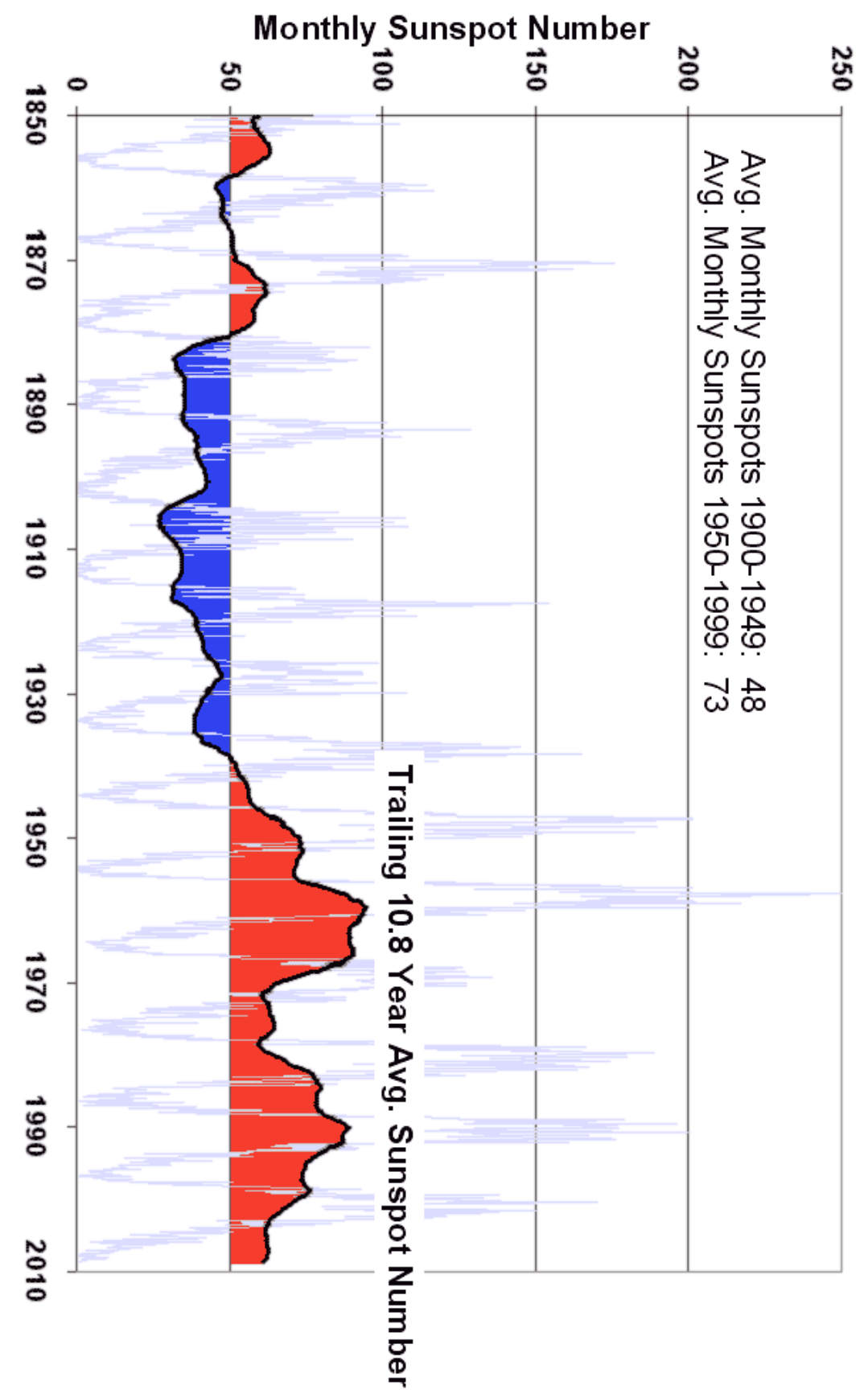

Figure 57: The number of Sunspots and the cool and warm periods in Europe. 


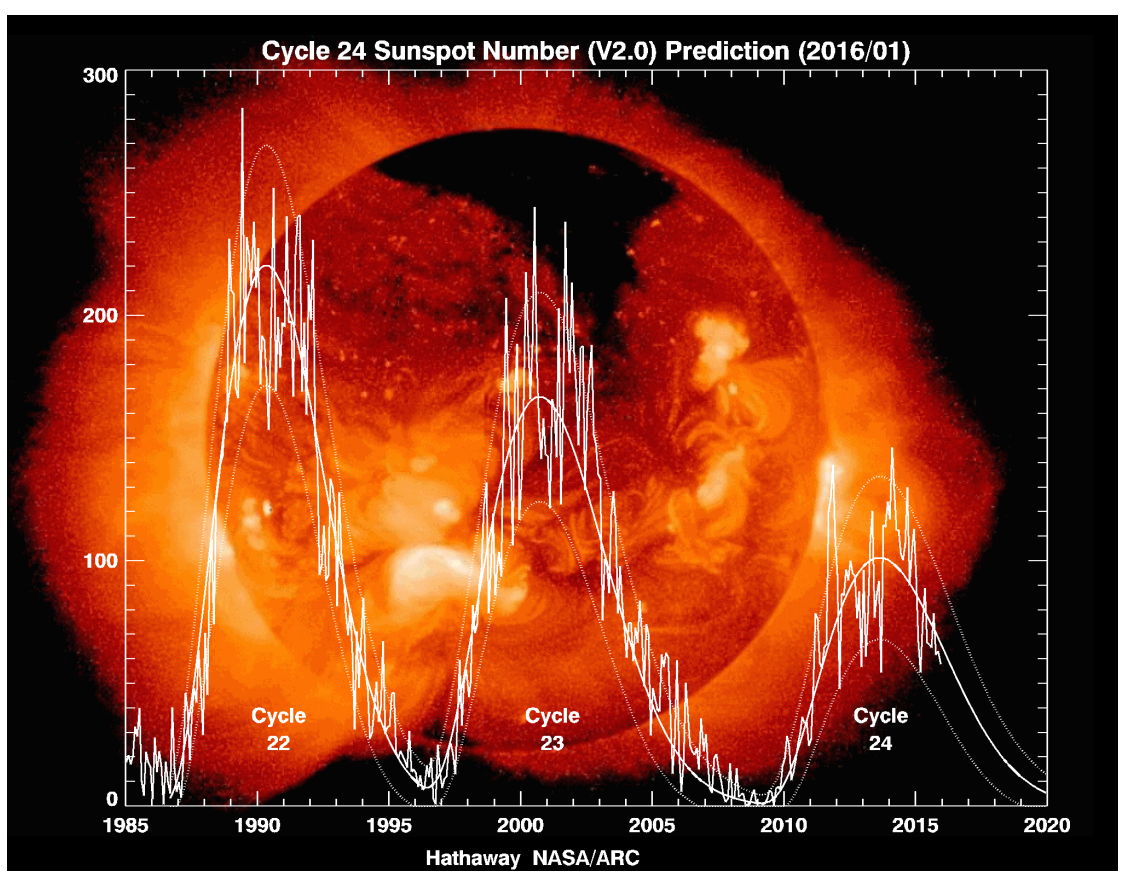

Figure 58: The 22nd, 23rd and slightly past the maximum of the 24th solar cycles, which the present one, shown on the background of the active Sun.

There are many efforts to explain the phenomenon but there is no prediction about its coming as there is no historical analogue about its past existence. It is for the first time that such a long 'pause' occurs. So what is unique in the past 18-19 years that gave rise to the pause? Note that the pause appears in the temperature but not in the concentration of any gas. This is an inverse argument usually applied to convince that $\mathrm{CO}_{2}$ is the culprit for Global Warming.

There are claims that one has to wait at least 30 years before declaring that the pause really means a halt in the Global Warming. We do not understand from where the 30 years time scale for such fluctuations is derived. In any case, if so we have to wait for another decade before deciding...

The plateau seems to agree with the fact that the current sunspots cycle is significantly lower than the previous one, which in turn is also lower from its predecessor. There are questions of what happened recently with this apparent pause in the global warming and we have to wait and see how the temperature will evolve.

So if one pole heats and not the other, we should look into the Hadley cell mechanism for answers.

\section{A small footnote}

We recall how for many years the IPCC advocated a wrong temperature distribution which exaggerates the rise of the temperature in recent years ${ }^{17}$.

\footnotetext{
${ }^{17}$ see Google climategate, http://www.climatedepot.com/2016/05/14/.
} 


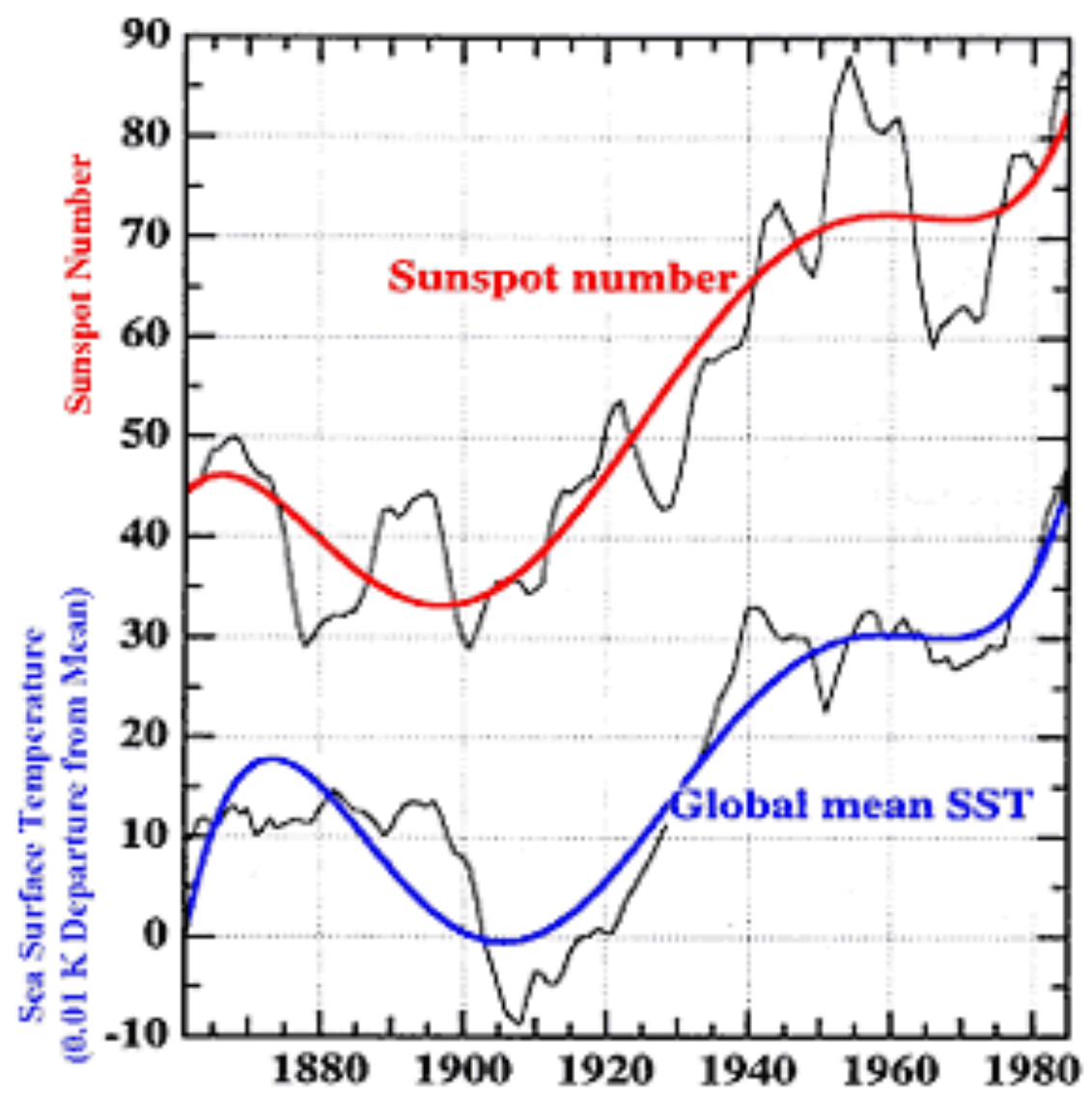

Figure 59: The correlation between the sunspot number and the Global Means of the Sea-Surface temperature.

\section{Acknowledgement}

It is my unique pleasure to thank professor Nir Shaviv from the Hebrew University and Dr. Smadar Bressler from the Technion, for multitudes of discussions about the content of this paper.

This work was supported by grant \#3-10413 from the Ministry of Science, Israel. 


\section{The period of the vertical motion is observed in the data}

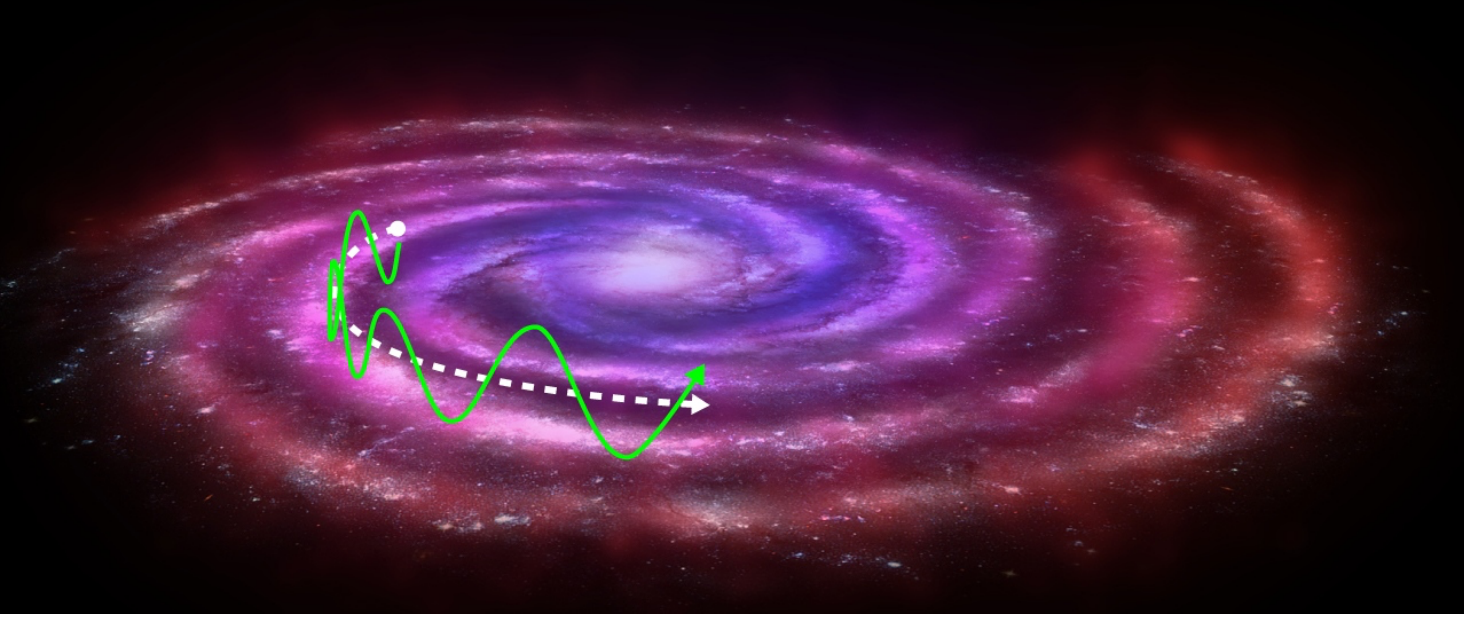

Figure 60: The change of the temperature over 32 million years caused by oscillations of the solar system perpendicular to the plane of the galaxy.

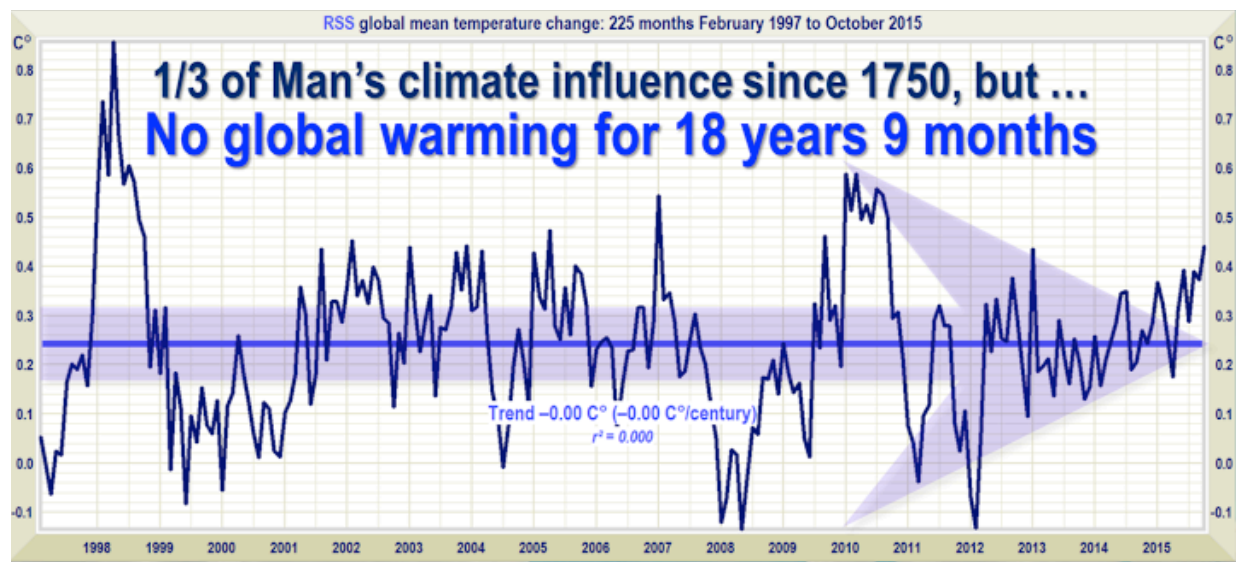

Figure 61: The temperature anomaly for the period of the so called 'pause'. 


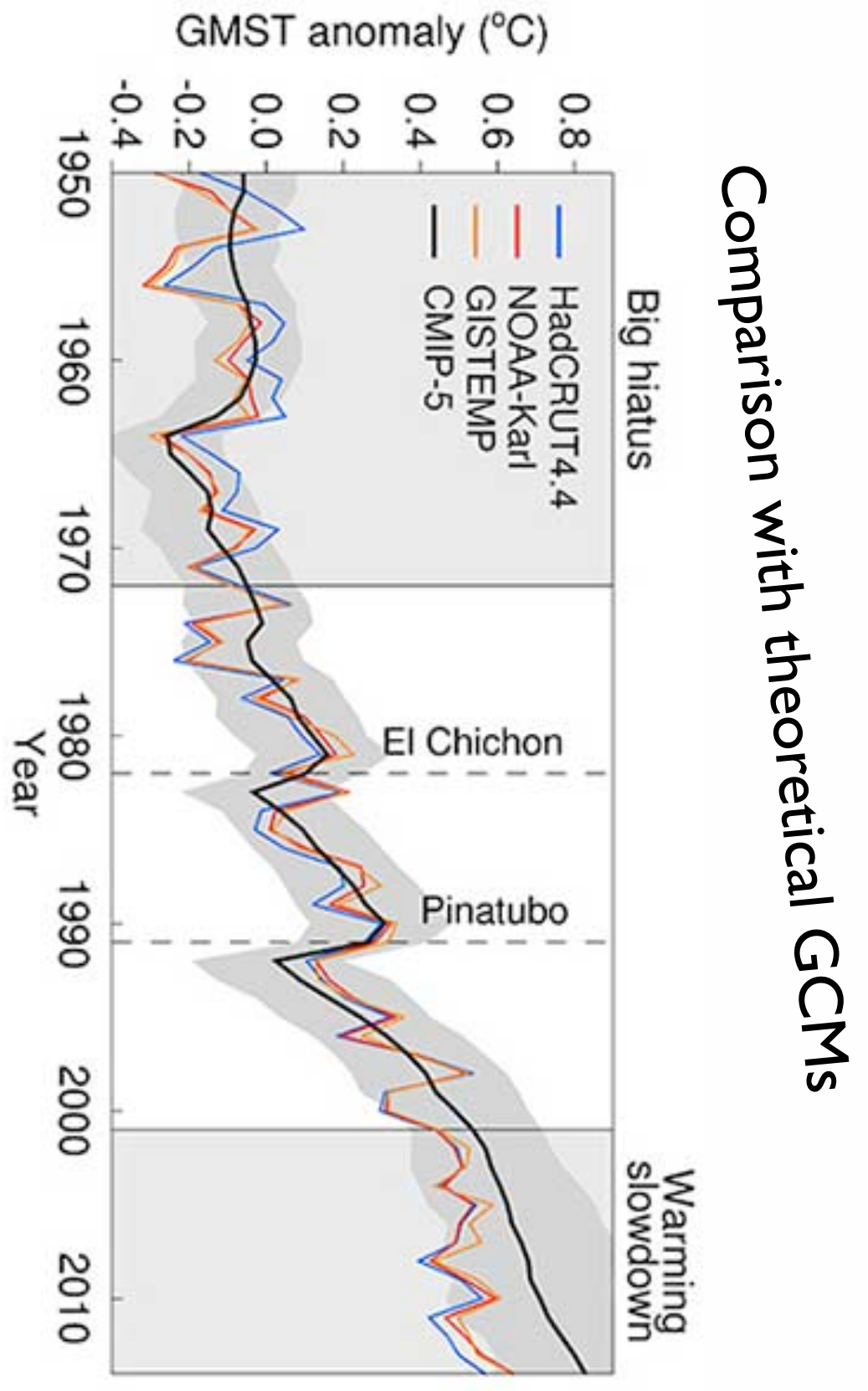

Figure 62: A comparison between the theoretical prediction and observation of the Temperature Anomaly starting from 1950. The 'pause' is recdeent in a more general perspective of time and magnitude. 


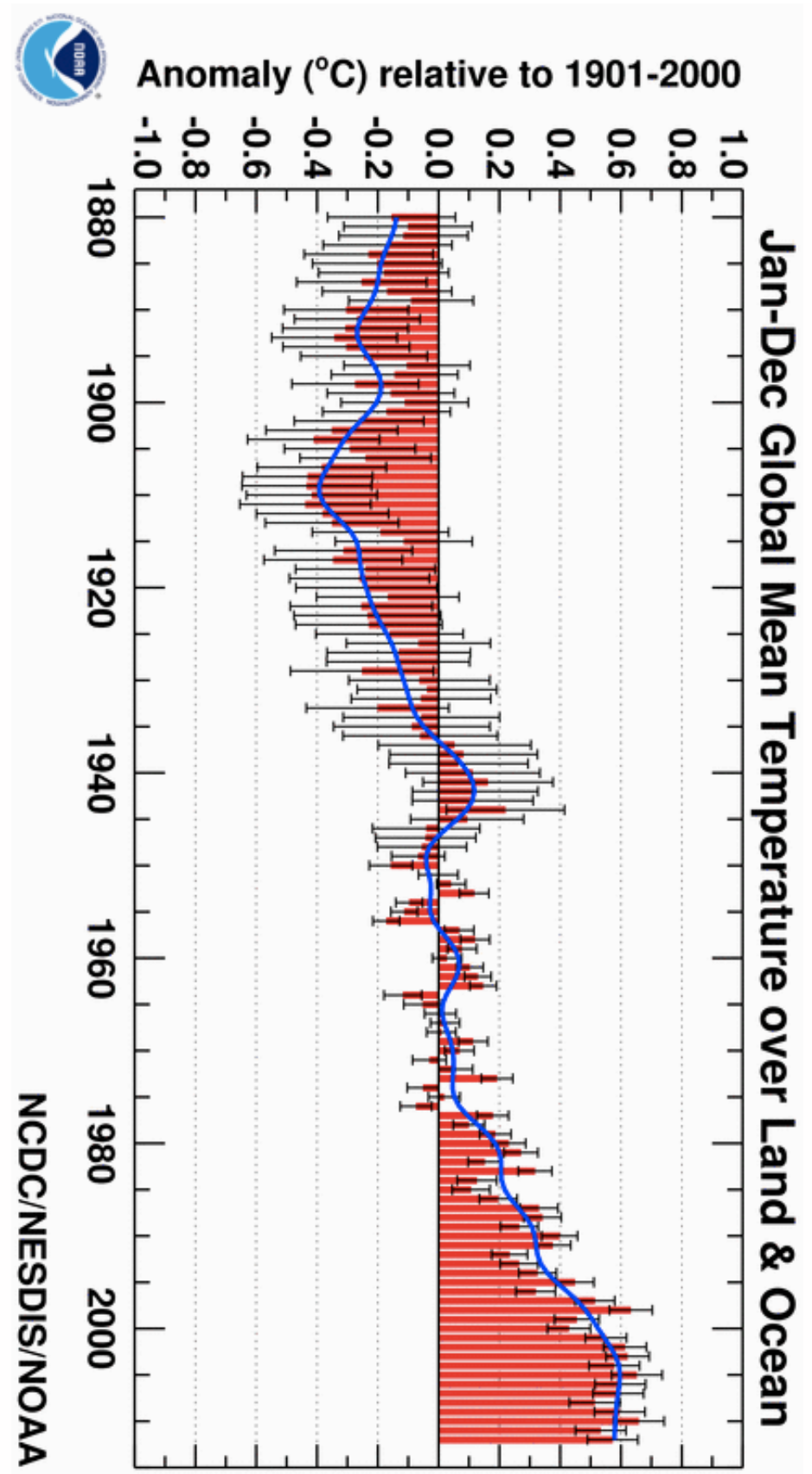

Figure 63: As above but starting at 1880. Here the 'pause' is seen as a slight decline. 


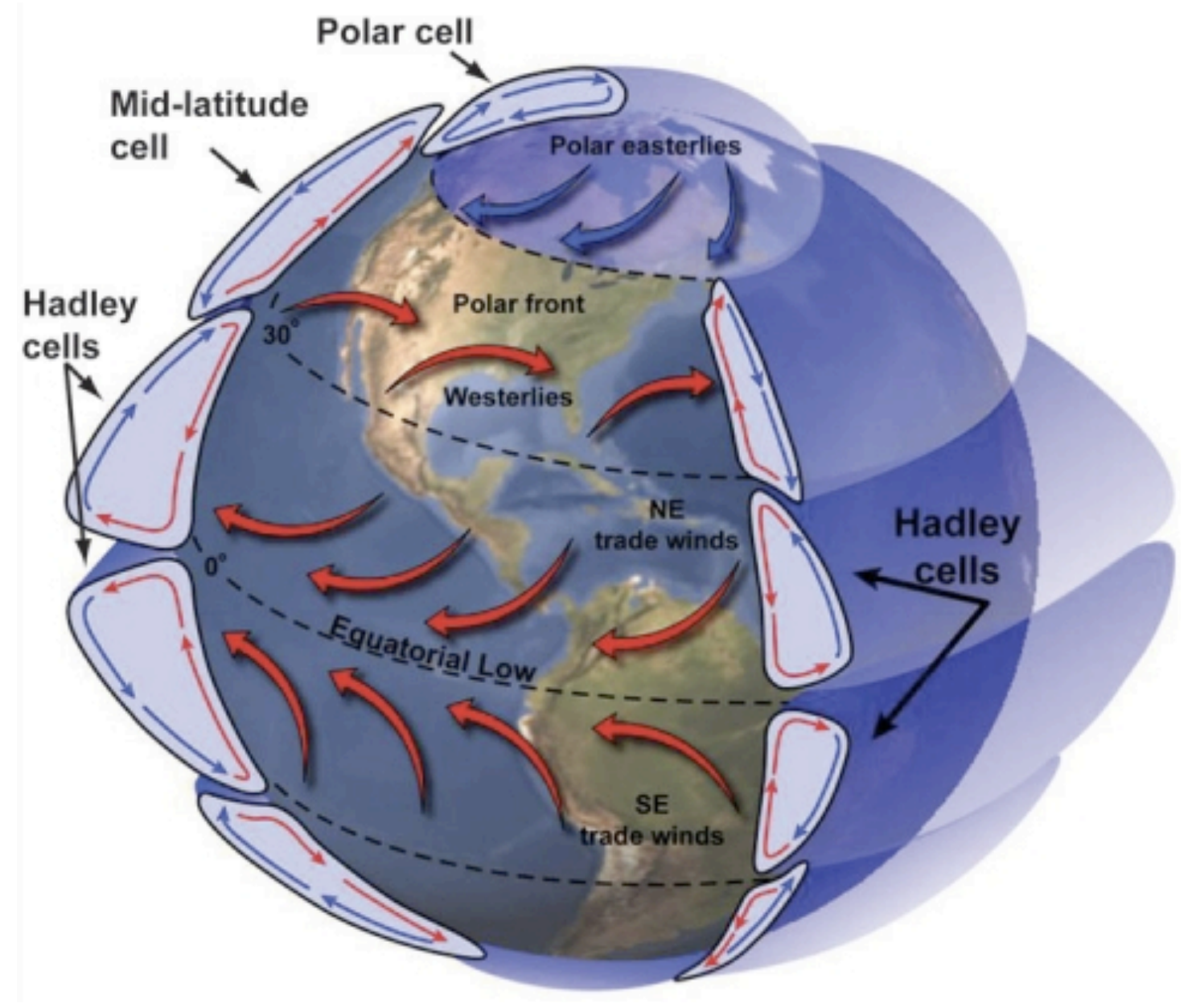

Figure 64: The general structure of the Hadley cell which carry heat from the equator to the poles. 


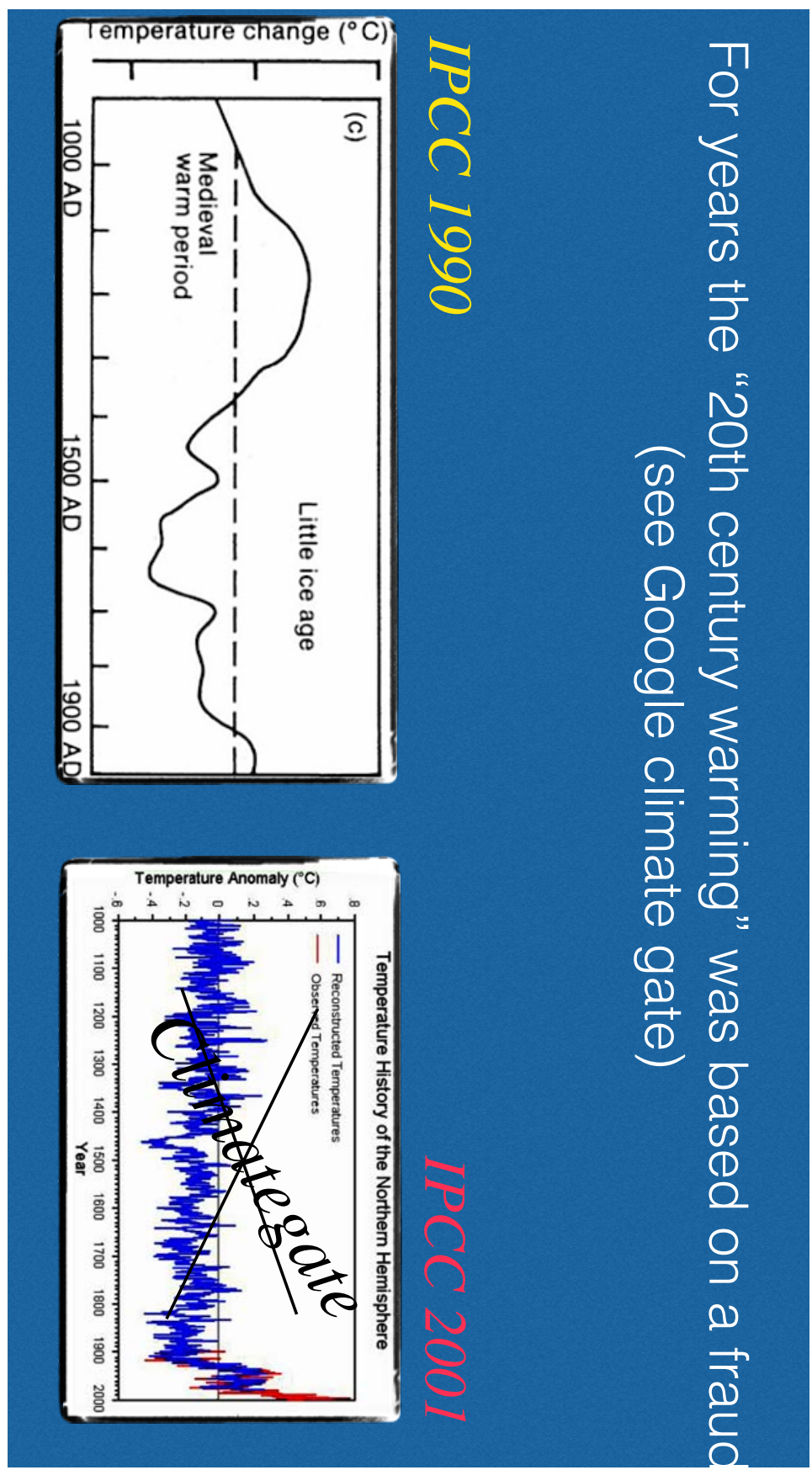

Figure 65: For many years the IPCC advocated a wrong temperature run with time known as the hockey stick. Getting reliable and trustful results is particularly important in a field like Global Warming where there are so many claims and data which is not always consistent with one another. 